\title{
Comparative proteogenomics deciphers the origin and evolution of eukaryotic chromatin
}

Authors: Xavier Grau-Bové ${ }^{1,2}$, Cristina Navarrete ${ }^{1,2}$, Cristina Chiva $^{2}$, Thomas Pribasnig ${ }^{3}$, Meritxell Antó $^{4}$, Guifré Torruella ${ }^{5}$, Luis Javier Galindo ${ }^{5}$, Bernd Franz Lang ${ }^{6}$, David Moreira ${ }^{5}$, Purificación López-Garcia ${ }^{5}$, Iñaki Ruiz-Trillo ${ }^{4,7}$, Christa Schleper ${ }^{3}$, Eduard Sabidó ${ }^{1,2}$, Arnau Sebé-Pedrós ${ }^{1,2 *}$

1. Centre for Genomic Regulation (CRG), Barcelona Institute of Science and Technology (BIST), Barcelona 08003, Catalonia, Spain

2. Universitat Pompeu Fabra (UPF), Barcelona 08003, Catalonia, Spain

3. Department of Functional and Evolutionary Ecology, Archaea Biology Unit, University of Vienna, Djerassiplatz 1, 1030 Vienna, Austria

4. Institut de Biologia Evolutiva (CSIC-Universitat Pompeu Fabra), Passeig Marítim de la Barceloneta, 37-49, Barcelona 08003, Catalonia, Spain.

5. Unité d'Ecologie Systématique et Evolution, CNRS, Université Paris-Saclay, AgroParisTech, Orsay, France

6. Department of Biochemistry, Robert Cedergren Centre, Université de Montréal, Montréal, Quebec H3C 3J7, Canada

7. ICREA, Pg. Lluís Companys 23, Barcelona 08010, Catalonia, Spain.

* Corresponding author: arnau.sebe@,crg.eu

\begin{abstract}
Histones and associated chromatin proteins have essential functions in eukaryotic genome organization and regulation. Despite this fundamental role in eukaryotic cell biology, we lack a phylogenetically-comprehensive understanding of chromatin evolution. Here, we combine comparative proteomics and genomics analysis of chromatin in eukaryotes and archaea. Proteomics uncovers the existence of histone post-translational modifications in Archaea. However, archaeal histone modifications are scarce, in contrast with the highly conserved and abundant marks we identify across eukaryotes. Phylogenetic analysis reveals that chromatin-associated catalytic functions (e.g., methyltransferases) have pre-eukaryotic origins, whereas histone mark readers and chaperones are eukaryotic innovations. We show that further chromatin evolution is characterized by expansion of readers, including capture by transposable elements and viruses. Overall, our study infers detailed evolutionary history of eukaryotic chromatin: from its archaeal roots, through the emergence of nucleosome-based regulation in the eukaryotic ancestor, to the diversification of chromatin regulators and their hijacking by genomic parasites.
\end{abstract}




\section{Introduction}

The access to genetic information in eukaryotes is controlled by a manifold nucleoproteic interface called chromatin. This nucleosomal chromatin environment defines a repressive ground state for transcription and other DNA-templated processes in eukaryotic genomes ${ }^{1,2}$. Multiple components associated with chromatin underlie elaborate eukaryotic genome regulation, allowing the differential access to genetic information in time/space and the maintenance of the resulting regulatory states ${ }^{3-6}$. Moreover, chromatin-based regulation is essential in repressing parasitic genomic elements, like transposons and viruses ${ }^{7-11}$.

The main protein component of eukaryotic chromatin are histones. All eukaryotes have four major types of histones (H2A, H2B, H3 and H4), which are combined as an octamer to form the basic repetitive unit of the chromatin: the nucleosome. Canonical histones are among the most highly conserved proteins across eukaryotes ${ }^{12}$ and, in addition, unique histone variants (paralogs of one of the four major histone types) are found in many species, often associated with particular regulatory states $^{13-16}$. Histone chemical modifications, including acetylations and methylations play a central role in genome regulation and transgenerational epigenetic inheritance ${ }^{3,17-20}$. These chemical moieties, known as histone post-translational modifications (hPTMs), are added and removed by specific enzymes ('writers', e.g., histone methyltransferases or acetylases; and 'erasers', e.g., histone demethylases and deacetylases). Some hPTMs (e.g., most acetylations) have a generic effect on nucleosome stability, while others are bound by specific proteins or protein complexes. These are often referred to as 'readers' and include proteins like HP1, which binds to $\mathrm{H} 3 \mathrm{~K} 9 \mathrm{me} 3$, as well as a myriad of other proteins encoding Chromo, PHD, Tudor and Bromo structural domains, among others ${ }^{21-23}$. Finally, nucleosome remodellers (like SNF2 proteins) and histone chaperones are additional important players in chromatin regulation, by mediating chromatin opening, nucleosomal assembly, and histone variant interchanges ${ }^{24-27}$.

All eukaryotes studied to date possess histone-based chromatin organization, with the sole exception of dinoflagellates, which nonetheless encode for histone proteins in their genomes ${ }^{28}$. Beyond eukaryotes, histones have also been identified in Archaea, where they have been shown to form nucleosomal structures ${ }^{29-32}$. However, unlike eukaryotic histones, the few archaeal histones experimentally characterized so far $(i)$ generally lack disordered $N$-terminal tails; ( $i i)$ do not have any known posttranslational modifications ${ }^{33}$; and (iii) do not seem to impose a widespread, genome-wide repressive transcriptional ground state ${ }^{32,34}$. Thus, chromatin-based elaborate genome regulation is often considered a eukaryotic innovation ${ }^{35,36}$.

From a phylogenetic perspective, our understanding of chromatin components and processes derives from a very small set of organisms, essentially animal, fungal and plant model species plus a few parasitic unicellular eukaryotes. Additional efforts have sampled specific aspects of chromatin regulation, such as histone modifications or their genome-wide distribution, in non-model animal species $^{37,38}$, fungi (Neurospora crassa and Fusarium graminearum) ${ }^{39,40}$, and five other eukaryotes: the unicellular holozoan Capsaspora owczarzaki ${ }^{41}$, the dinoflagellate Hematodiunium sp. ${ }^{28}$, the brown algae Ectocarpus siliculosus ${ }^{42}$, the amoebozoan Dictyostelium discoideum ${ }^{43}$, and the ciliate Tetrahymena thermophila ${ }^{44,45}$. However, these organisms represent a tiny fraction of eukaryotic diversity. 
Hence, we lack a systematic understanding of the evolution of eukaryotic chromatin modifications and components ${ }^{46}$.

In order to infer the origin and evolutionary diversification of eukaryotic chromatin, we performed a joint comparative analysis of histone proteomics data from 30 different eukaryotic and archaeal taxa, including new data for 23 species. In parallel, we analyzed the complement of chromatin-associated gene families in an additional 172 eukaryotic genomes and transcriptomes. This comprehensive taxon sampling includes representatives of all major eukaryotic lineages, as well as multiple free-living members of enigmatic early-branching eukaryotes (e.g., jakobids, malawimonads, Meteora sp., ancyromonads and CRuMs; Fig. 1a). In addition, in order to trace the pre-eukaryotic origins of these chromatin gene families, we systematically searched for orthologs in archaeal, bacterial and viral genomes. Specifically, we reconstructed the evolutionary history of enzymes involved in chromatin modification and remodelling; as well as the conservation of the hPTMs effected by these enzymes. Our comparative genomics and proteomics suggest a concurrent and early origin of canonical histones, a core of quasi-universal hPTMs, and their corresponding enzymatic effectors. We also identify independent expansions in hPTM reader gene families across eukaryotes and document evidence of the capture of these reader domains by parasitic genomic elements. Overall, this work provides a phylogenetically-informed framework to classify and compare chromatin components across the eukaryotic tree of life, and to further investigate the evolution of hPTM-mediated genome regulation.

\section{Results}

\section{Comparative proteomics of eukaryotic histone post-translational modifications.}

We analyzed the phylogenetic distribution and evolutionary history of histone proteins. To this end, we surveyed the presence of histone-fold proteins across 172 eukaryotic and 4,226 archaeal taxa, using HMM searches (Fig. 1a,b and Supplementary Table 1). Histone proteins are found in all eukaryotic genomes. We clustered the identified 8,576 histone-encoding proteins using pairwise local alignments and then classified individual sequences in these clusters based on pairwise alignments to a reference database ${ }^{47}$ (Fig. 1a and Supplementary Fig. 1b). This reveals four broad clusters corresponding to the four main eukaryotic histones (H2A, H2B, H3, and H4) and their variants (H2AZ, macroH2A, and CENP), as well as a fifth cluster composed of archaeal HMfB homologs. Finally, this classification also uncovers three large connected components composed of transcription factors with histone-like DNA binding domains, which are widely distributed in eukaryotes (POLE3, POLE4, DR1) and/or archaea (NFYB). Further analysis of the genomic distribution of these histone genes shows a frequent occurrence of $\mathrm{H} 3-\mathrm{H} 4$ and $\mathrm{H} 2 \mathrm{~A}-\mathrm{H} 2 \mathrm{~B}$ pairs in head-to-head orientation (5' to $5^{\prime}$ ), strongly indicating co-regulation across eukaryotes (Supplementary Fig. 1e,f).

Next, we investigated the distribution and conservation of hPTMs across major eukaryotic groups and Archaea, including methylations, acetylations, crotonylations, phosphorylations, and ubiquitylations. To this end, histones from 19 different eukaryotic species were extracted, chemically derivatized $^{48}$ and analyzed by mass-spectrometry (Fig. 1c and Supplementary Table 3), adding to previously available hPTM proteomics data for additional seven species. Our extensive taxon sampling covers all major eukaryotic groups, as well as hitherto unsampled early-diverging eukaryotic line- 
ages - such as the malawimonad Gefionella okellyi, the discoban Naegleria gruberi, or the ancyromonad Fabomonas tropica - , thus providing a comprehensive comparative framework for evolutionary inference.

We focused first on hPTMs present in canonical histones, as defined by their highly conserved $N$ terminal regions, phylogenetic analyses, and sequence similarity to curated reference canonical histones (Fig. 1d and Supplementary Fig. 1b; see Methods). hPTMs are detected in all canonical histones from all species. After correcting by sequence coverage, we observe that hPTMs are particularly abundant in $\mathrm{H} 3$ canonical histones (median $=23.5 \mathrm{hPTMs}$ per species, mean $=24.3$ ), compared with H2A, H2B and H4 (medians between 6.5 and 9, means between 9.5 and 13.4; Supplementary Fig. 1b). Holozoan canonical H2As (Homo sapiens, Sycon ciliatum and Capsaspora owczarzaki) represent an exception to this trend and contain similar number of modifications to H3s in these species (Supplementary Fig. 1b).

Canonical $\mathrm{H} 3$ and $\mathrm{H} 4 \mathrm{~N}$-terminal tails contain the majority of phylogenetically-conserved hPTMs, in stark contrast with the relative paucity of conserved hPTMs in canonical H2A and H2B. A striking example of paneukaryotic conservation comes from the acetylation of the H4 K5, K8, K12 and K16 residues (Fig. 1d, second panel), all of which mark gene expression-permissive chromatin environments in multiple eukaryotic species ${ }^{21}$. A similar conservation pattern is observed in the acetylation of a group of $N$-terminal H3 lysines (K9, K14, K18, K23, K27) associated with similar functions, while other $\mathrm{H} 3$ acetylations are only found in a few species (e.g., residues K4, K56 and K79). While acetylations are highly conserved, only seven histone $\mathrm{H} 3 / \mathrm{H} 4$ methylations are broadly conserved across eukaryotic lineages: H3K4me1/2/3, H3K9me1/2/3, H3K27me1/2/3, H3K36me1/2/3, H3K37me1/2/3 and, more sparsely, H3K79me1/2 and H4K20me1. Many of these broadly conserved marks have conserved roles in demarcating active (e.g., $\mathrm{H} 3 \mathrm{~K} 4 \mathrm{me}$ ) and repressive chromatin states (e.g., H3K9me and H3K27me) ${ }^{21,41,49}$. The scarcity of conserved hPTMs in H2A and H2B canonical histones can partially explained by their higher degree of sequence divergence (Fig. 1e), which is reflected in many non-homologous lysine residues (Fig. 1d). But even among homologous positions, we found little evidence of conservation, with the exception of H2A K5ac (associated to active promoter ${ }^{50}$ ) and, in fewer species, methylation of H2A K5 and H2B K5. Finally, we were also able to identify phosphorylations in serine and threonine residues and a few instances of ubiquitylation. In general, these marks show more restricted phylogenetic distributions than lysine acetylation or methylation, even in the tightly conserved $\mathrm{H} 3$ and $\mathrm{H} 4$ histones. We can identify conserved phosphorylations in H2A T120 and S122, which are shared by most opisthokonts, and the ubiquitylation of H2A K119 only in some holozoan species.

Mass-spectrometry analysis detected histone variants in all species included in our study, suggesting that they are relatively abundant in the chromatin of these eukaryotes (Fig. 1e and Supplementary Fig. 1c). Most of these variants are lineage-specific, with the exception of the paneukaryotic variants H2AZ, H3/CENBP and H3.3; and the macroH2A variant found in holozoans and Meteora sp. (belonging to an orphan eukaryotic lineage). Interestingly, we find hPTMs in the vast majority of detected variants, both conserved and lineage-specific, particularly acetylations and methylations (Fig. 1e and Supplementary Fig. 1c). Overall, our comparative proteomic analysis suggests the existence of a highly conserved set of canonical hPTMs of ancestral eukaryotic origin in $\mathrm{H} 3$ and H4, which co- 
exists with less conserved hPTMs in H2A, H2B, and lineage-specific modifications in variant histones.

\section{Archaeal histones and histone post-translational modifications}

In contrast with the paneukaryotic distribution of histones, sequence searches show that only a fraction of archaeal genomes encode for histones (28.1\% of the taxa here examined; Fig. 2a). Archaeal histones exhibit a patchy phylogenetic distribution, similar to other gene families shared with eukaryotes $^{51}$. Among others, histones are present in Euryarchaeota, the TACK superphylum and Asgard archaea - the latter representing the closest known archaeal relatives of eukaryotes ${ }^{12,52-55}$. This extended sampling revealed that Asgard archaea histones, particularly in the Lokiarchaeota and Heimdallarchaeota clades ${ }^{54}$, often have lysine-rich $N$-terminal tails in the manner of eukaryotic histones (Fig. 2a-c). These Asgard histones appear to be conserved across multiple taxa, albeit without direct sequence similarity compared to canonical eukaryotic histones (Supplementary Fig. 1d and Supplementary Table 2). When compared with eukaryotic sequences, these archaeal histones cluster separately and are most similar to either $\mathrm{H} 4$ or, to a lesser degree, $\mathrm{H} 3$ canonical histones, in line with previous findings ${ }^{12,54,56}$.

To identify potential archaeal hPTMs, we performed proteomics analysis of histones in three Euryarchaeota (the Methanobacteriota Methanobrevibacter cuticularis and the Halobacteriota Methanospirillum stamsii and Methanosarcina spelaei) and one Thaumarchaeota species (Nitrososphaera viennensis; Fig. 2b). Mass-spectrometry detects histone proteins in all of them: 2-4 in the euryarchaeotes (with $27-90 \%$ protein coverage) and one in the thaumarchaeote (80\% protein coverage), including homologs with $N$-terminal tails encoded by each of the three euryarchaeotes in our survey (22-40 aa, 0.09-028 lysines per residue; Fig. 2c). Moreover, this proteomics analysis finds evidence of hPTMs in archaeal histones. However, in comparison with eukaryotic histones, hPTMs are extremely scarce in archaeal histones. Specifically, we identify no hPTMs in N. viennensis and M. spelaei (one and two histones detected, respectively), three acetylations and one methylation in M. stamsii (in three out of four histones detected), and one acetylation and two methylations in M. cuticularis (in two out of four histones; Fig. 2b, top). Interestingly, we find conserved lysine residues with shared modifications in M. stamsii and M. cuticularis (methylation in K54 and acetylation in K57; Fig. 2b, bottom). This result indicates that highly-abundant hPTMs represent a eukaryotic innovation, likely linked to dynamic nucleosomal regulation in eukaryotes but not in Archaea.

\section{Taxonomic distribution of chromatin-associated proteins}

hPTMs are deposited and removed by specific modifying enzymes ('writers' and 'erasers'), while 'reader' protein domains found in diverse proteins bind and recognize specific hPTMs. For example, Bromo and Chromo domains bind acetylated and methylated lysine residues, respectively. In addition, the control of histone loading/eviction from specific genomic loci is mediated by chromatin remodellers, like SNF2 proteins ${ }^{26}$, and histone chaperones ${ }^{25}$. To date, the classification and evolutionary analysis of this chromatin machinery has been based on biased, partial taxonomic samplings 
and has not employed phylogenetic methods ${ }^{57}$ (with rare exceptions ${ }^{12,26}$ ), often resulting in inaccurate orthologous relationships and confounded classification and naming schemes.

We sought to obtain a systematic, phylogenetics-based classification of histone remodellers, chaperones, readers, and modifiers in order to understand the evolutionary history of eukaryotic chromatin (Fig. 3a). To this end, we ( $i$ ) compiled a taxa-rich dataset of 172 eukaryotic genomes and transcriptomes, covering all major eukaryotic supergroups and devoting particular attention to early-branching, non-parasitic lineages (Fig. 1a and Supplementary Table 1), as well as genomic data from 4,226 Archaea, 24,886 Bacteria and 185,579 viral taxa; (ii) defined a protein structural domain as a proxy for each gene family (Supplementary Table 4) and retrieved all genes in these genomes that contained these domains; and (iii) inferred accurate orthology groups from phylogenetic analyses of each gene class (next section).

We examined the taxonomic distribution and abundance of the major gene classes (Fig. 3b,c). Many domains with chromatin-associated functions in eukaryotes are also present in Archaea and Bacteria, albeit with scattered phylogenetic distributions (Fig. 3b and Supplementary Fig. 2a,b). Families with prokaryotic homologs include mostly catalytic gene classes (writer, eraser and remodeller enzymes), whereas readers and histone chaperones are virtually absent from prokaryotes (Fig. 3b and Supplementary Fig. 2a,b). Histone fold-encoding genes constitute a case in point for this patchy distribution of chromatin proteins in prokaryotes: they are present in most archaeal phyla, but are absent in about half of the sampled genomes within each (Fig. 3b). Yet, there is a qualitative difference between the phylogenetic distribution of archaeal and bacterial chromatin-associated gene classes: whereas archaeal histones tend to co-occur with chromatin-associated gene classes, the bacterial complement of writers and erasers is much less conserved and is uncorrelated with the extremely rare presence of histone-like genes (Fig. 3d and Supplementary Fig. 2b).

Within eukaryotes, most gene structural classes associated with chromatin functions are ubiquitously distributed across all lineages here surveyed, supporting an early eukaryotic origin for the core chromatin machinery (Fig. 3b and Supplementary Fig. 2d). In fact, the total number of chromatin writer, eraser and remodeller enzymes remains remarkably stable across eukaryotes (Fig. 3e). The only exception is the marked increase in genes encoding reader domains observed in lineages exhibiting complex multicellularity: animals, streptophyte plants, and, to a lesser degree, phaeophyte brown algae (Stramenopila). This occurs partially due to the addition of new gene classes (e.g., SAWADEE in the Plantae s.l. + Cryptista lineage, or ADD_DNMT3 in bilaterians and cnidarians), but also via the expansion of ancient, widely-distributed reader gene classes (e.g., Tudor, PHD, Chromo or Bromo domains Supplementary Fig. 2d). These taxonomic patterns indicate that chromatin modifying and remodelling catalytic activities originated in prokaryotes, while reader and chaperone structural domains are eukaryotic innovations.

\section{Phylogenetics of chromatin modifiers and remodellers}

To gain detailed insights into the origin and evolution of chromatin gene families, we used phylogenetic analysis to define orthology groups from paneukaryotic gene trees. We surveyed 172 eukaryotic species and defined a total of 1,713 gene families (orthogroups) encompassing 51,426 genes, 95\% of which were conserved in two or more high-ranking taxonomic groups (as listed in Fig. 1a), and which 
included 51,426 genes in total (Supplementary Table 5). We annotated each gene family according to known members from eukaryotic model species. For simplicity, we use a human-based naming scheme throughout the present manuscript (unless otherwise stated), but we also provide a dictionary of orthologs in three additional model species (Arabidopsis thaliana, Saccharomyces cerevisiae and Drosophila melanogaster; see Supplementary Table 5). This phylogenetic classification scheme of eukaryotic chromatin gene families, as well as the sequences and associated phylogenetic trees, can be explored and retrieved in an interactive database: https://sebe-lab.shinyapps.io/chromatin_evolution

We first investigated the potential pre-eukaryotic origins of these gene families/orthogroups by comparing their phylogenetic distance to prokaryotic sequences and to other eukaryotic orthogroups (Fig. 4a). Most eukaryotic gene families are more closely related to other eukaryotes than to prokaryotic sequences, supporting the idea that writers, erasers, remodellers and readers diversified within the eukaryotic lineage, as previously noted for histones ${ }^{12}$. This analysis also reveals a substantial fraction of eukaryotic gene families with close orthogroups in Archaea and Bacteria, which pinpoints components that were $(i)$ inherited from a prokaryotic ancestor during eukaryogenesis; (ii) laterally transferred between eukaryotes and prokaryotes at later stages; or (iii) a combination of both phenomena. For example, we identified a sister-group relationship between the eukaryotic SIRT7 deacetylase and a clade of Asgard archaea Sirtuin enzymes (Heimdallarchaeota and Lokiarchaeota), a topology consistent with an archaeal origin; whereas SIRT6 appears nested within other eukaryotic sequences (Fig. $\mathbf{4 b}$, left panel). Likewise, the KAT14 acetylase is more closely related to bacterial enzymes than to other eukaryotic acetylases (Fig. 4b, right).

Next, we mapped the phylogenetic distribution of orthogroups in order to infer the origin and diversification of individual chromatin gene families (Fig. 4c and Supplementary Fig. 3a). Using probabilistic inference of ancestral gene content, we reconstruct a rich Last Eukaryotic Common Ancestor (LECA) complement of chromatin-associated gene families: 65 acetylases (amongst which 61 were conserved in at least two of the most deeply sampled eukaryotic early-branching lineages, namely Amorphea, Diaphoretickes, and Discoba); 20 deacetylases (19 in these early-branching eukaryotic lineages); 59 methyltransferases (55); 42 demethylases (38); 33 remodellers (33); and 25 chaperones (18) (Fig. 4c and Supplementary Table 5). The subsequent evolution of these families is characterized by relative stasis, with few new orthologous families emerging in later-branching eukaryotic lineages. Notable exceptions include the origin of KAT5 deacetylases and KMT5B/C SET methyltransferases in Opisthokonta; KAT8 and SIRT7 in Holozoa; and Viridiplantae-specific deacetylases (homologs of $A$. thaliana HDA7 and HDA14 deacetylases) and SETs (A. thaliana PTAC14); among others.

In spite of their broad distributions across eukaryotes, many chromatin modifier families exhibit variation in their protein domain architectures, likely conferring them functional properties such as distinct binding preferences (Supplementary Fig. 3b). For example, most CREBBP/EP300 acetylases consist of a catalytic HAT_KAT11 domain and two TAZ and ZZ zinc finger domains, but different lineages have acquired different reader domains: an acetylation-reading Bromo domain in holozoans and stramenopiles, PHD in plants and some stramenopiles, and no known reader domains in other lineages (e.g., in the fungal orthologs of the S. cerevisiae protein RTT109). A similar pattern is apparent in SET methyltransferase families sharing a core catalytic domain (SET) harboring variable 
DNA- and chromatin-interacting domains - animal SETDB1/2 homologs have MBD domains that bind CpG methylated DNA, while plants have SAD_SAR domains with the same function; and holozoan ASH1L homologs encode Bromo and BAH readers, whereas phaeophytes encode PHD domains (Supplementary Fig. 3b). Other architectures, however, are much more conserved, as exemplified by the presence of Tudor-knot and MYST zinc finger domains in most KAT5 deacetylases; or the ubiquitous co-occurrence of Helicase-C and SNF2_N domains in most remodellers (Supplementary Fig. 3b).

Specific examples of evolutionarily conserved chromatin gene families include the catalytic core and the subunits of well-studied chromatin complexes like PRC1 (RING1/AB, PCGF), PRC2 (EZH1/2, SUZ12, EED, RBBP4/7) and Trithorax/MLL (MLL1/2/3/4, WRD5, ASH2L, RBBP5, DPY-30; Fig. 4d,e). However, when we compared the distribution of these complexes with the hPTMs they are related to, we found a generally poor co-occurrence (Fig. 4f-h). For example, organisms like Dictyostelium discoideum and Creolimax fragrantissima lack EZH1/2 orthologs, but we detected H3K27me3 in these species; while Thecamonas trahens and Naegleria gruberi lack Dot1 orthologs but have H3K79me marks. A poor correlation is also observed between the occurrence of H3K9me and that of SUV39H1 orthologs. An exception to this pattern is the ubiquitous distribution of H4K16ac and the acetylase family KAT5/ $8^{58}$ (Fig. 4h). These patterns suggest that the specificity between hPTMs and their writers might not be completely conserved across eukaryotes, with distinct members of the same gene classes (e.g., methyltransferases) performing similar roles. In this context, reading domains present in writing/erasing enzymes (directly in the same protein or as part of multiprotein complexes) are likely to play a major role in the re-purposing of chromatin catalytic activities.

\section{Evolutionary expansion of chromatin readers}

Multiple protein structural domains have been involved in the recognition of hPTMs, such as Bromo and PHD domains binding to acetylated lysines or Chromo, MBT and Tudor domains binding to methylated lysines ${ }^{22,23}$. These are generally small domains and can be found both as stand-alone proteins as well as in combination with other domains, often catalytic activities such as hPTM writers, erasers and remodellers. Thus, they are central in the establishment of functional connections between chromatin states. To understand the contribution of these reading domains to the evolutionary diversification of chromatin networks, we studied in detail the phylogeny and protein architecture of reader domains across eukaryotes.

We quantified the co-occurrence frequency of reader and catalytic domains, finding $(i)$ that most reader domains are present in genes without writer, eraser or remodeller domains $(87 \%$, Fig. 5a and Supplementary Fig. 4a); and (ii) that most cases of reader-catalytic co-occurrence involve PHD, Chromo and Bromo domains (Supplementary Fig. 4a). For example, the conserved architecture of the paneukaryotic CHD3/4/5 re-modellers includes Chromo readers in most species and PHD domains specifically in animals and plants (Supplementary Fig. 3b). Likewise, PHD domains are often present in the KMT2A/B and KMT2C/D SET methyltransfrase; and the ASH1L family has recruited Bromo and BAH domains in holozoans, and PHD in multicellular stramenopiles (Supplementary 
Fig. 3b). In spite of these redundancies, reader families typically have independent evolutionary histories, as illustrated by the fact that most reader domain-containing genes encode only one such domain (92\%, Supplementary Fig. 4b).

We next performed phylogenetic analyses of individual reader domains and reconstructed the gains and losses of these reader gene families/orthogroups (Fig. 5a). Compared to the relative stasis of catalytic enzyme families, this reader-centric analysis revealed a strikingly different evolutionary pattern of lineage-specific bursts of innovation, particularly amongst PHD, Chromo and Bromo genes, as well as Tudor in animals (Fig. 5a and Supplementary Fig. 4c). PHD, Chromo and Bromo families also appeared as the most abundant in the reconstructed LECA reader domain repertoire, which amounted to 89 gene families (Fig. 5a, left). The distribution of gene family ages in extant species also corroborates that readers have emerged at evolutionarily more recent nodes of the tree of life than catalytic gene families (Fig. 5b).

\section{Co-option of the chromatin machinery by transposable elements}

Further examination of the domain co-occurrence networks of readers revealed that Chromo and PHD domains are often present together with protein domains found in transposable elements (TEs; Fig. 5c and Supplementary Table 6), including retrotransposons (e.g., retrotranscriptases and integrases; orange modules in Fig. 5c) and DNA transposons (e.g., DNA binding domains and transposases; red modules). It is known that some TEs show insertion-preferences associated to specific chromatin states $^{59}$, often mediated by direct chromatin tethering mechanisms ${ }^{60}$. For example, the Chromo domain of the MAGGY gypsy retrotransposon of the fungus Magnaporthe grisea targets H3K9me regions ${ }^{61}$. Reciprocally, some protein domains of TE origin, often DNA-binding domains, have been co-opted into chromatin and transcriptional regulators ${ }^{62}$. Thus, we decided to explore in detail the occurrence of chromatin-associated domain (readers, but also catalytic domains) linked to TEs in the 172 eukaryotic genomes in our dataset (Fig. 5d). Moreover, we used available RNA-seq datasets in many of these species to validate some of these TE fusions (Fig. 5d-e). A fully validated fusion gene would $(i)$ come from a non-discontinuous gene model in the original assembly, and (ii) have evidence of expression, with reads mapping along the entire region between the TE-associated domain and the chromatin-associated domain.

We identified 823 predicted gene models containing both chromatin- and TE-associated domains (Fig. 5d). Whilst these TE fusions were not exclusive of reader domains, most such fusions involved PHD and Chromo-encoding genes; followed by SNF2_N remodellers, SET methyltransferases, and others. An homology search against a database of eukaryotic TEs revealed that most of these candidate TE fusions could be aligned to known retrotransposons or DNA transposons. For example, by way of validation, our analysis identifies the SETMAR human gene, a previously-described fusion between a SET methyltranferase and a Mariner-class DNA transposon ${ }^{63}$. Overall, $31 \%$ of the candidate fusion genes were supported by valid gene models according to our stringent criteria (Fig. 5d). Interestingly, we find very few cases of hypothetical fusions between TEs and Bromo domains, which recognize K acetylations and are otherwise highly abundant across eukaryotes (Supplementary Fig. 4b), and none of them is validated by RNA-seq data. This could be explained by the detrimental effect 
of targeting TE insertions to sites of active chromatin demarcated by histone acetylations, such as promoter and enhancer elements.

Some of these validated fusions have a broad phylogenetic distribution (Fig. 5e), such as a GypsyERV retrotransposon with a $C$-terminal Chromo domain (Unk. Chromo 2.1 in Fig. 5e) that is widely distributed in animals and various microbial eukaryotes, and contains dozens of paralogs in vertebrate Danio rerio or the charophyte Chara braunii, many of which are expressed. Another widespread Gypsy-ERV retrotransposon with a Chromo domain is present in multiple expressed and highly similar copies in the fungi Rhizopus delemar (Fig. 5f,e), suggesting a successful colonization of this genome by this TE. By contrast, other TE fusions are taxonomically restricted to one or few related species, such as the fusion of hAT activator DNA transposons with Chromo CBX and CDY readers in the sponge Ephydatia muelleri; or multiple instances of fusions with Chromo and PHD readers in cnidarians. A common fusion in cnidarians involves different retrotransposon classes with PHD domains orthologous to the PYGO1/2 protein (Fig. 5e), which is known to recognize specifically $\mathrm{H} 3 \mathrm{~K} 4 \mathrm{me}^{64}$. Another particularly striking case is that of the box jellyfish Morbakka virulenta, where we identified fusions between TEs and the catalytic SNF2 domains of various known families of readers (CHD1/2, TDRD6/15, MORC1/2/3/4), remodellers (INO80, SMARCAD1, ERCC6L, RAD54B/L), and chromatin acetylases (KAT2A/B). Globally, this analysis reveals that recruitment of chromatin reading and even modifying domains by TE has occurred in many eukaryotic species, in a way that might facilitate the evasion from suppressing mechanisms in the host genomes as suggested by the expansion of Chromo-fused TEs in the genomes of Chara braunii (Viridiplantae), Chromera velia (Alveolata) and Rhizopus delemar (Fungi).

\section{Chromatin components in viral genomes}

In addition to TEs, chromatin is also involved in the suppression of another type of genomic parasites: viruses. Some chromatin-related genes, including histones, have been found in viral genomes, especially among the nucleocytoplasmic large DNA viruses - also known as giant viruses. Eukaryotic core histones have been even hypothesized to have evolved from giant virus homologs, after the discovery that certain Marseilleviridae genomes encoded deeply-diverging orthologs of the four canonical histones ${ }^{65}$. These viral histones have been recently shown to form nucleosome-like particles that package viral DNA 66,67 .

We analyzed the distribution and abundance of chromatin-related protein domains among viruses, including data from 1,816 giant virus genomes. Based on structural domain searches, we identified 2,163 viral chromatin-related proteins (Fig. 5g and Supplementary Table 6). The majority of these proteins are encoded by giant viruses (55\%), followed by Caudovirales (37\%). Among these two groups, only giant virus genomes encode histones - specifically, the Iridoviridae, Marseilleviridae, Mimiviridae, Pithoviridae, and Phycodnaviridae families. Concordantly with previous studies ${ }^{68}$, we also identify remodellers in all giant virus families; as well as less abundant components of the chromatin writer/eraser/reader toolkit (Fig. 5g).

We then investigated the phylogenetic affinities of these viral chromatin proteins, starting with histones (Fig. 5h). Our analysis recovers the phylogenetic affinity of Marseilleviridae histones with specific eukaryotic histone families ${ }^{65}$, and makes this pattern extensive to Mimiviridae, Iridoviridae, and 
Pithoviridae giant viruses (Fig. 5h), with the caveat of the ambiguous clustering of the H4-like viral histones with either $\mathrm{H} 4$ eukaryotic or archaeal HMfB genes. In all these lineages, we identify genes encoding two histone-fold domains orthologous to H2B + H2A (inset table in Fig. 5h), whereas the H4 + H3 histone doublet genes appears to be exclusive to Marseilleviridae. By contrast, histone homologs in Phycodnaviridae, Pandoraviridae (also giant viruses), and Polydnaviridae (incertae sedis) are never found as either doublets or as early-branching homologs of eukaryotic histones, suggesting recent acquisition from eukaryotes.

Unlike histones, most of the viral chromatin-associated genes exhibited a mixture of prokaryotic and eukaryotic phylogenetic affinities (Fig. 5i and Supplementary Fig. 6a-d) and often lack affinity to any specific eukaryotic gene family (Supplementary Fig. 6e). Viral readers, on the other hand, are often embedded within eukaryotic clades in gene trees and are similar to bona fide eukaryotic families, exhibiting topologies consistent with recent, secondary acquisitions. This is the case of BIRC2/3/XIAP readers widespread in the Baculoviridae, which encode BIR domains that are often hijacked from their hosts ${ }^{69}$. We also find a number of viral Chromo-encoding genes, which fall in two main taxonomic categories: (i) giant virus homologs of the eukaryotic CBX1/3/5 family (present in Mimiviridae, Iridoviridae and Phyodnaviridae); and (ii) homologs from various Adintoviridae, which are closely related to animal Chromo genes encoding rve integrase domains ${ }^{70}$ (Fig. 5i and Supplementary Table 6). Finally, we also identify a handful of eukaryotic-like viral genes with deep-branching positions relative to core eukaryotic gene families, as seen in histones (Fig. 5h). This includes Mimiviridae homologs of the eukaryotic methyltransferases SMYD1-5 and DOT1 (Supplementary Fig. 6b,c), as well as SNF remodeller families with homologs in distinct giant virus clades (HLTF/TTF2 in Phycodnaviridae, Mimiviridae and Iridoviridae). These results indicate that cases of horizontal transfer from eukaryotes to viruses are common in different chromatin-related gene families, including histones. Therefore, it is likely that basally-branching giant virus histones were similarly acquired from a stem eukaryotic lineage and this would explain the observed histone tree topology with extant eukaryotic species. In any case, most of the eukaryotic chromatin machinery appears to have cellular roots.

\section{Discussion}

Our comparative proteogenomics study reconstructs in detail the origin and evolutionary diversification of eukaryotic chromatin components, from post-translational modifications to gene family domain architectures. We looked first at the pre-eukaryotic roots of chromatin. Multiple aspects of archaeal chromatin have been studied in recent years, including nucleosomal patterns ${ }^{30}$ and the structure of the archaeal nucleosome ${ }^{29}$. A recent taxonomic survey of archaeal nucleoid-associated proteins revealed multiple independent diversifications of DNA-wrapping proteins and a strong association between high levels of chromatinization and growth temperature, overall suggesting a structural, nonregulatory role for archaeal chromatin ${ }^{71}$. Our proteomics data support this notion by showing the scarcity of hPTMs in four species belonging to two different archaeal lineages (Euryarchaeota and Thaumarchaeota). An earlier proteomics study reported the complete absence of hPTMs in the euryarchaeote Methanococcus jannaschii ${ }^{33}$, but here we do identify a few instances of modified lysine residues in Euryarchaeota. It remains to be seen if hPTMs are frequently present in Asgard and other unsampled archaeal linages, where other eukaryotic-like features have been found ${ }^{72-74}$. In fact, some 
of these Asgard, particularly Lokiarchaeota, encode for histones with long, K-rich $N$-terminal tails but that bear no similarity with eukaryotic histones and are, therefore, most probably the result of convergent evolution. Interestingly, Lokiarchaeota genomes also frequently encode histone modifiers such as SET methyltransferases and MOZ_SAS acetylases. However, overall our results suggest that extensive usage of hPTM is a eukaryotic innovation (Fig. 6a). Similarly, while we find the majority of catalytic domains of hPTM writers, hPTM erasers and chromatin remodellers in Archaea and even Bacteria, these appear only scattered in a small fraction of the examined taxa. In contrast, hPTM reader domains and histone chaperones are eukaryotic innovations, further supporting the idea that the functional readout of hPTMs and the role for histone variants in defining chromatin states are both exclusive to eukaryotes (Fig. 6a).

The origin of eukaryotes represents a major evolutionary transition in the history of life ${ }^{75}$. Thanks to sequencing and comparative analysis of archaeal and eukaryotic genomes, we also have a detailed reconstruction of the massive innovation in gene repertoires that occurred at the origin of eukaryotes. This gene innovation in the Last Eukaryotic Common Ancestor (LECA) includes cytoskeletal proteins and associated motors like myosins ${ }^{76,77}$ and kinesins ${ }^{78}$, vesicle trafficking apparatus ${ }^{79}$, splicing machinery ${ }^{80}$, ubiquitin signalling systems ${ }^{81}$ and a large repertoire of sequence-specific transcription factors $^{36}$. Combining parsimony analysis and knowledge on gene function in extant lineages (mostly vertebrates, yeast and plants), our results allow us to reconstruct a complex LECA repertoire of hPTMs and associated writing, eraser and reader gene families (Fig. 6b,c). We infer 23 to 29 highlyconserved lysine acetylations in canonical histones (e.g., H3K9ac and H3K27ac) and a repertoire of 65 and 20 histone acetylase and deacetylase families, respectively. With the exception of H4K16ac ${ }^{58}$, most histone acetylations are thought to exert a generic, perhaps additive, effect on the opening of chromatin $^{21}$. As such, acetylation marks like H3K27ac have been found to be enriched in promoters of active genes in diverse eukaryotes ${ }^{41}$. In contrast, histone methylations often have very specific readouts and they can be linked both to active and repressive chromatin states. We infer between 13 and 25 conserved methylated lysine residues in LECA histones, including marks typically associated to active promoters (H3K4me1/me2/me3), gene bodies (H3K36me3, H3K79me1/2, H4K20me1), and repressive chromatin states (H3K9me2/me3, H3K27me3, H4K20me3) ${ }^{82,83}$. Finally, we also infer the existence of two histone variants in the LECA (H2AZ and H3.3), as well 33 chromatin remodellers (e.g., EP400/SWR1 and INO80, involved in loading and removal of H2AZ, respectively) and 25 histone chaperones (e.g., ASF1A/B and NPM1/2/3). This indicates that, in addition to an extensive repertoire of hPTMs, the regulation of nucleosomal histone composition was also an important feature in the LECA.

Chromatin evolution after the origin of eukaryotes is characterized by an expansion of lineage-specific histone variants harboring unique hPTMs and a net expansion in the number of reader gene families, as opposed to the relatively static catalytic gene families (writers, erasers and remodellers). This is particularly relevant as it suggests extensive remodelling of chromatin networks during eukaryote evolution, that is, changes in the coupling of particular hPTMs to specific functional chromatin states. An example of such changing state-definitions comes from looking at the hPTMs associated to TEs in different organisms: H3K9me3+ H4K20me3 in animals, H3K27me3 in some plants ${ }^{84}$, H3K79me2+H4K20me3 in the brown multicellular algae Ectocarpus siliculosus ${ }^{42}$, and H3K9me3+H3K27me3 in the ciliate Paramecium tetraurelia ${ }^{85}$. In the context of the histone code 
hypothesis ${ }^{3,19,86-88}$, our findings indicate that, while there is an ancient core of conserved hPTMs across eukaryotes, evidence for a universal code/functional-readout is limited, with perhaps the exception of the highly conserved configuration of ancient hPTMs around active promoters across many eukaryotes ${ }^{41}$. Another interesting observation related to the evolution of chromatin networks is the capture of chromatin proteins (usually reader domains) by TEs. We find evidence of this phenomenon in a number of species with a scattered phylogenetic distribution, suggesting that it is a recurrent process and that it often leads to the successful propagation of the TE in the host genome. We hypothesize that this process facilitates the targeting of TEs to specific chromatin states, although some of these fusion TEs may also be involved in the modification of the chromatin environment of the TEs, as it has been described in the case of DNA methylation capture by TEs ${ }^{89,90}$.

In the future, a broader phylogenetic understanding of the genome-wide distribution of hPTMs, as well as the direct interrogation of hPTM binders in different species ${ }^{91-93}$, will be crucial to further clarify questions such as the ancestral role of specific hPTM and the co-option of ancient hPTMs into novel functions.

\section{Acknowledgements}

We want to thank Josep Casacuberta for Physcomitrella patens samples, Harold J. G. Meijer for Phytophthora infestans samples, Maja Adamska for Sycon ciliatum samples, and Alistar Simpson for access to the Gefionella okellyi culture (made possible by his funding from NSERC, Canada). Research in A.S-P. group was supported by the European Research Council (ERC) under the European Union's Horizon 2020 Research and Innovation Programme Grant Agreement (851647), the Spanish Ministry of Science and Innovation (PGC2018-098210A-I00), the Centro de Excelencia Severo Ochoa scheme (SEV-2016-0571), and the Agencia Estatal de Investigacion. C.N. is supported by an FPI PhD fellowship from the Spanish Ministry of Economy, Industry and Competitiveness (MEIC). X.G-B. is supported by a Juan de la Cierva fellowship (FJC2018-036282-I) from the Spanish Ministry of Economy, Industry and Competitiveness (MEIC). I.R-T. was supported by a European Research Council Grant (616960). B.F.L. was supported by the Natural Sciences and Engineering Research Council of Canada (NSERC; RGPIN- 2017-05411) and by the 'Fonds de Recherche Nature et Technologie', Quebec. P.L-G. and D.M. were supported by a Moore and Simons foundations grant (GBMF9739) and by European Research Council Advanced Grants (322669, 787904). Research in C.S. group was supported by the European Research Council (ERC) through project TACKLE (AdvGrant No. 695192).

\section{Author contributions}

A.S.-P. conceived the project. X.G.-B., C.C., I.R.T., C.S., E.S. and A.S.-P. designed experiments and analytical strategies. C. N., T.P., M. A. and A.S.-P. performed experiments. X.G.-B., C.C., and A.S.-P analyzed the data. T.P., G.T., L.J.G., D.M.P.L-G. and B.F.L. provided biological samples/cultures and genomic data. All authors contributed to data interpretation. X.G.-B. and A.S.-P. wrote the manuscript with input from all authors.

\section{Declaration of interests}

The authors declare no competing interests. 
bioRxiv preprint doi: https://doi.org/10.1101/2021.11.30.470311; this version posted December 1, 2021. The copyright holder for this

preprint (which was not certified by peer review) is the author/funder, who has granted bioRxiv a license to display the preprint in

Figure 1 perpetuity. It is made available under aCC-BY-NC 4.0 International license.

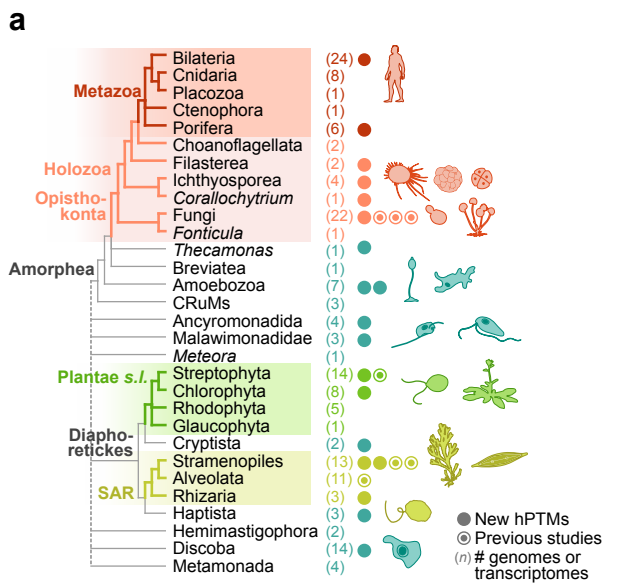

b
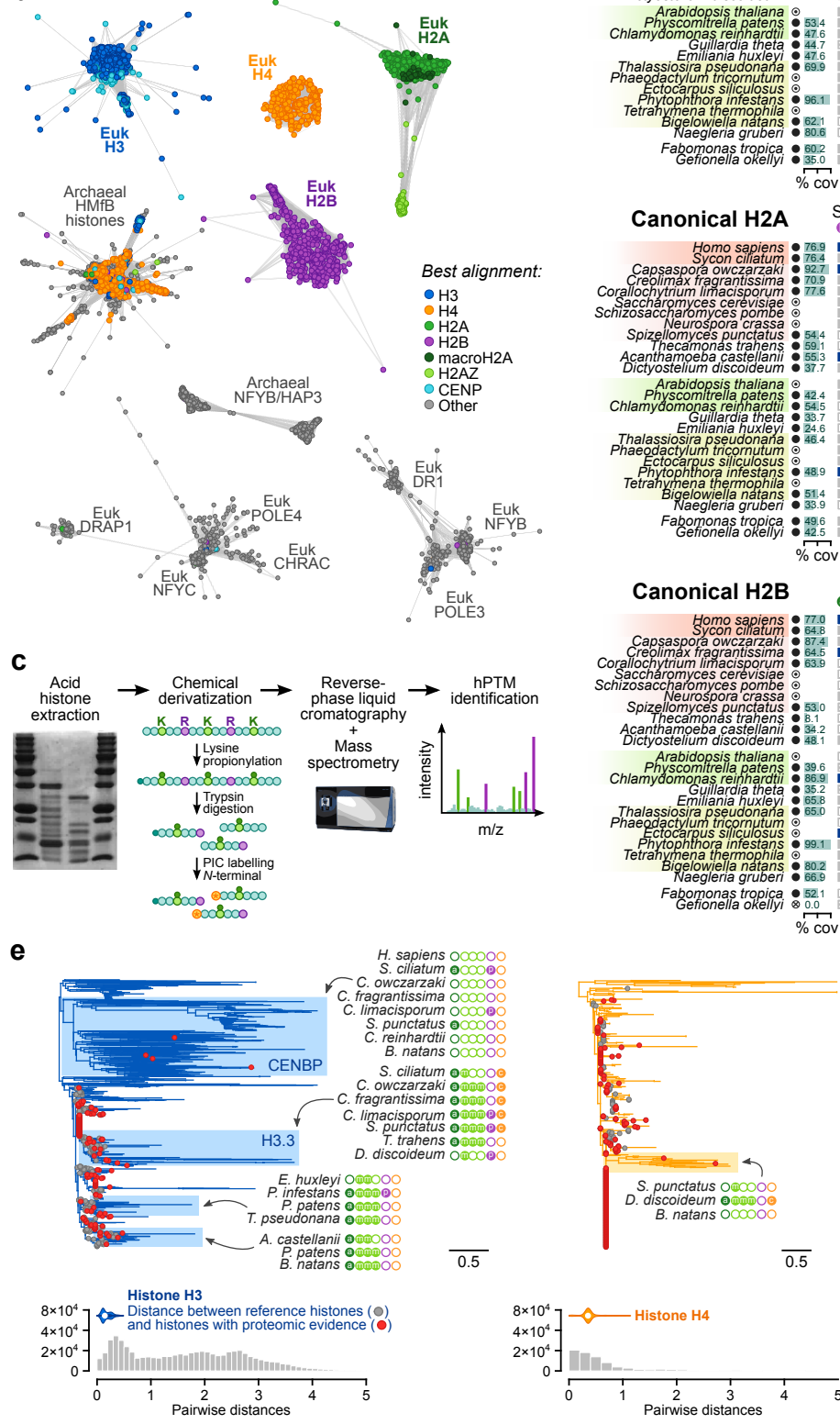

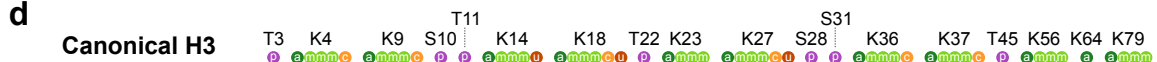
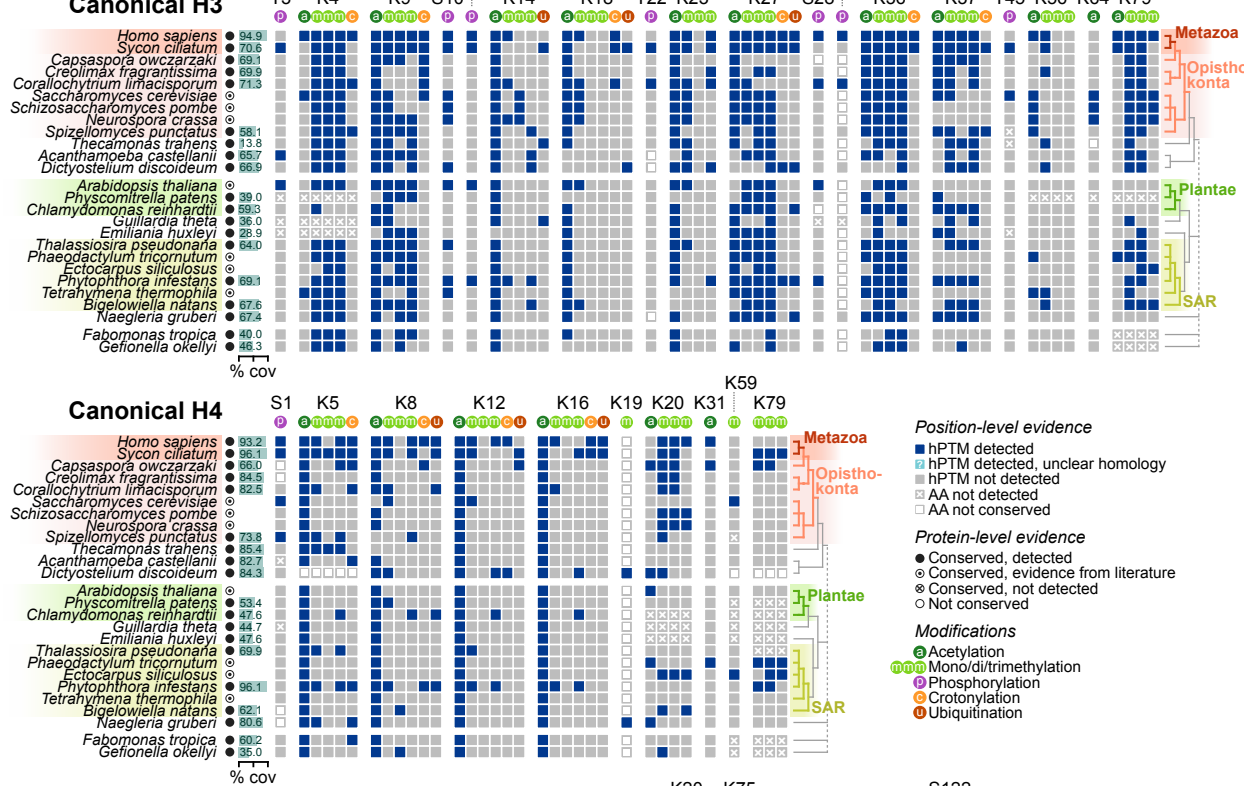

Position-level evidence

HPTM detected
RPTM detected, unclear homology $\mathrm{hPTM}$ not detected
$\mathrm{AAA}$ not detected

Protein-level evidence - Conserved, detected
- Conserved, evidence from literature Conserved, not detected
Not conserved Modifications EAcetylation
(1) Monoldiltrimethylation
OPhosphorylation EProstonhlyation
@Ubiquitination $\mathrm{S} 122$
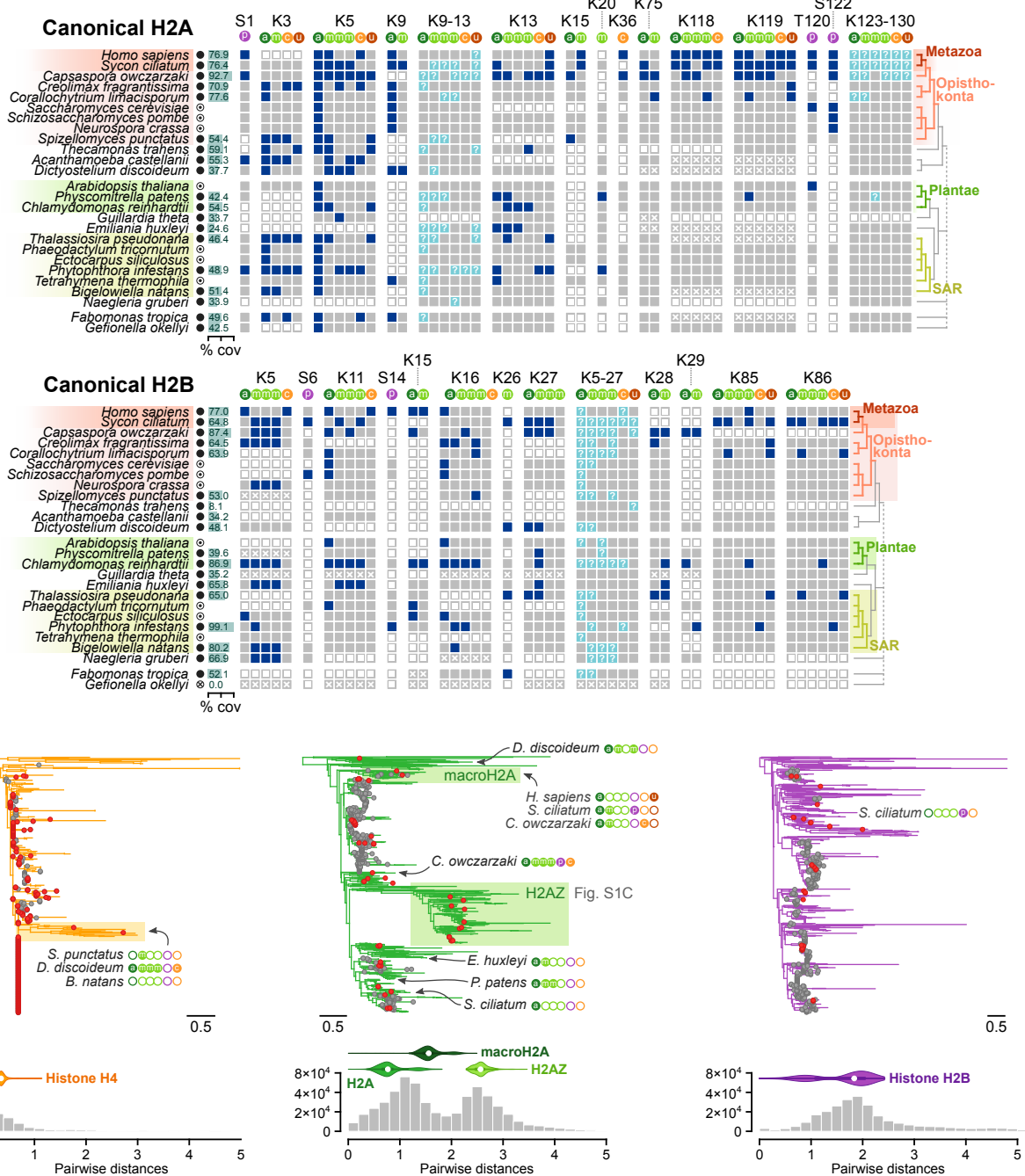
Figure 1. Diversity of post-translational modifications in eukaryotic canonical and variant histones. a, Eukaryotic taxon sampling used in this study. Coloured dots indicate the number of species used in the comparative histone proteomics reconstruction, with solid dots indicating new species added in this analysis. Numbers in brackets indicate the number of genomes/transcriptomes used in the comparative genomics analyses. Dashed lines indicate uncertain phylogenetic relationships. Complete list of sampled species in Table S1. b, Networks of pairwise protein similarity between histone protein domains in eukaryotes, archaea and viruses. Each node represents one histone domain, coloured according to their best alignment in the HistoneDB database (see Methods). Edges represent local alignments (bitscore $\geq 20$ ). c, Schematic representation of the hPTM proteomics analysis strategy employed in this study. $\mathbf{d}$, Conservation of hPTM in eukaryotic histones. hPTM coordinates are reported according to the amino-acid position in human orthologs (if conserved). In $\mathrm{H} 2 \mathrm{~A}$ and $\mathrm{H} 2 \mathrm{~B}$, question marks indicate the presence of hPTMs in stretches of lysine residues of uncertain homology. Only positions with hPTMs conserved in more than one species are reported (full table and consensus alignments available in Table S3). e, Top, Maximum likelihood phylogenetic trees of the connected components in (B) corresponding to eukaryotic histones (H3, H4, H2A, H2B). Canonical histones included in (D) and variant histones with observed hPTMs are highlighted. Bottom, distributions of pairwise phylogenetic distances between all proteins in each gene tree. Violin plots above each distribution represent the distribution of distances between reference histones present in the HistoneDB database and histones with proteomic evidence included in our study, for each of the main canonical (H3, H4, H2A, and H2B) and variant histones (H2AZ and macroH2A). 
bioRxiv preprint doi: https://doi.org/10.1101/2021.11.30.470311; this version posted December 1, 2021. The copyright holder for this

preprint (which was not certified by peer review) is the author/funder, who has granted bioRxiv a license to display the preprint in

\section{Figure 2} perpetuity. It is made available under aCC-BY-NC 4.0 International license.

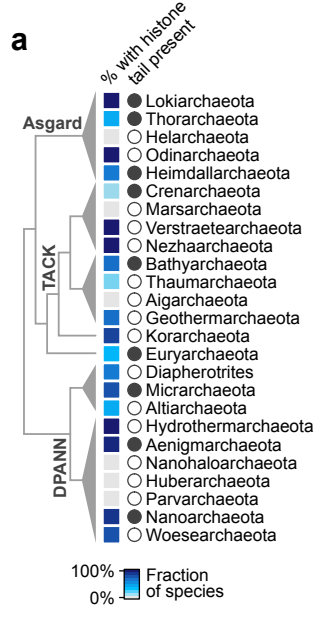

b

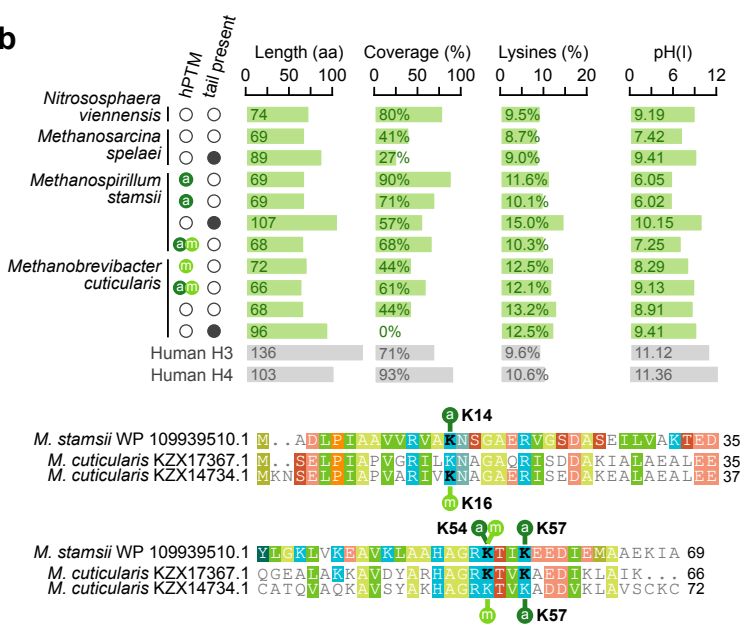

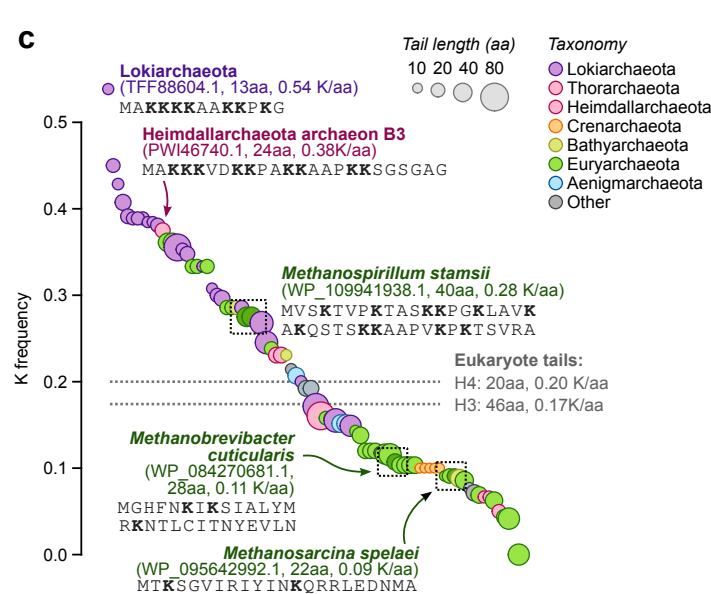

d
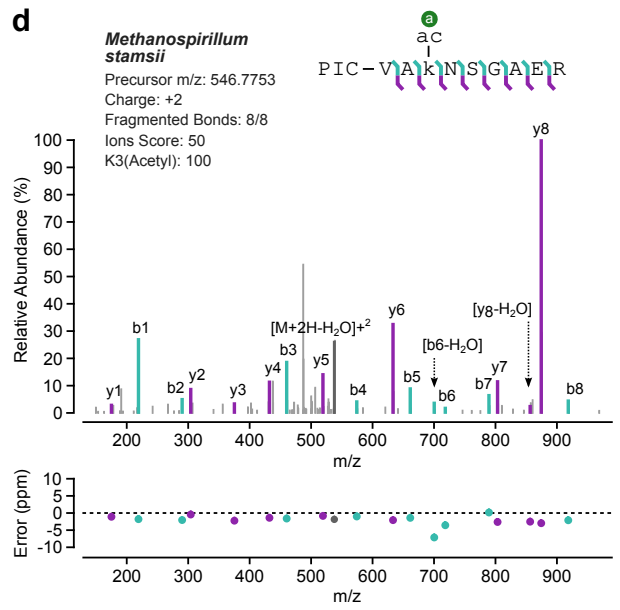
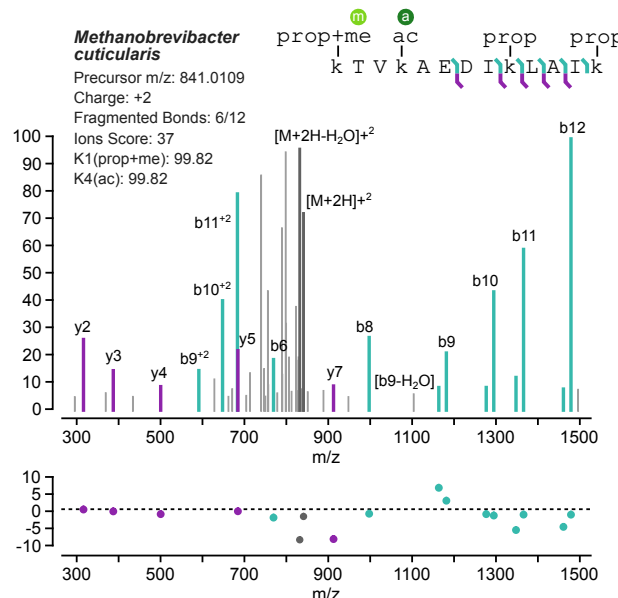
Figure 2. Archaeal histone diversity and post-translational modifications. a, Distribution of histones (fraction of taxa in each lineage) and histone tails (presence/absence) across Archaea phyla. b, Summary of proteomics evidence of archaeal histones, including the presence of modifications, tails, coverage, fraction of lysines identified, and isoelectric points. Human Histone H3 and H4 are included for reference. The alignments at the bottom depict the position of lysine modifications in the globular part of Methanospirillum stamsii and Methanobrevibacter cuticularis HMfB histones (modified residues in bold). c, Archaeal HMfB histones with $N$-terminal tails (at least 10 aa before a complete globular domain), sorted by frequency of lysine residues in the tail and colour-coded according to taxonomy (same as panel A). Amino-acid sequences shown for selected examples. The dotted line indicates the median frequency of lysines in canonical eukaryotic $\mathrm{H} 3$ and $\mathrm{H} 4$ histone tails. Source data available in Table S2. d, Mass spectra of three modified archaeal peptides, representing the relative abundance of fragments at various mass-to-charge ratios $(\mathrm{m} / \mathrm{z})$. Spectra were annotated using IPSA. $b$ and y ions and their losses of $\mathrm{H}_{2} \mathrm{O}$ are marked in green and purple, respectively; precursor ions are marked in dark grey. Unassigned peaks are marked in light grey. Some labels have been omitted to facilitate readability. 
bioRxiv preprint doi: https://doi.org/10.1101/2021.11.30.470311; this version posted December 1, 2021. The copyright holder for this

preprint (which was not certified by peer review) is the author/funder, who has granted bioRxiv a license to display the preprint in

Figure 3 perpetuity. It is made available under aCC-BY-NC 4.0 International license.

a

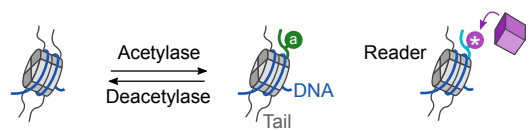

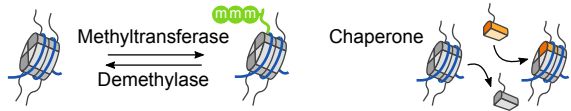

Remodeller

C

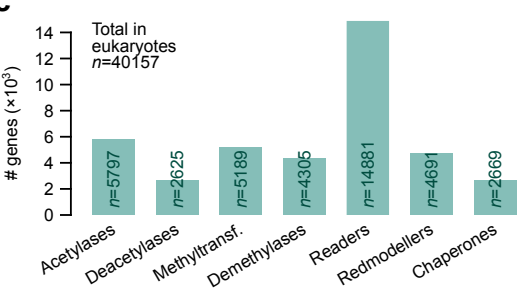

d

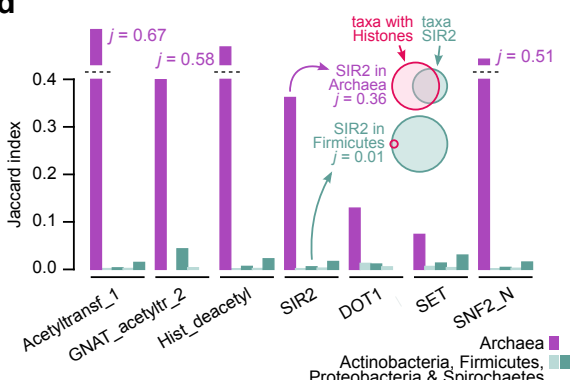

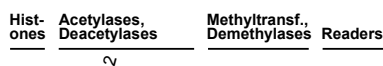
Remodeller $\perp$ Histone chaperones

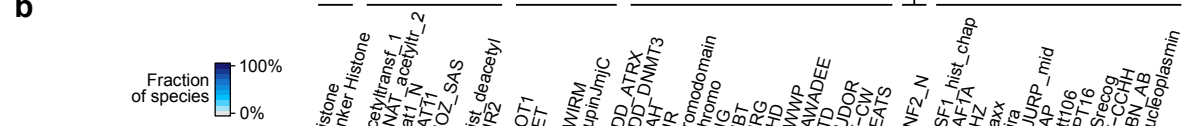

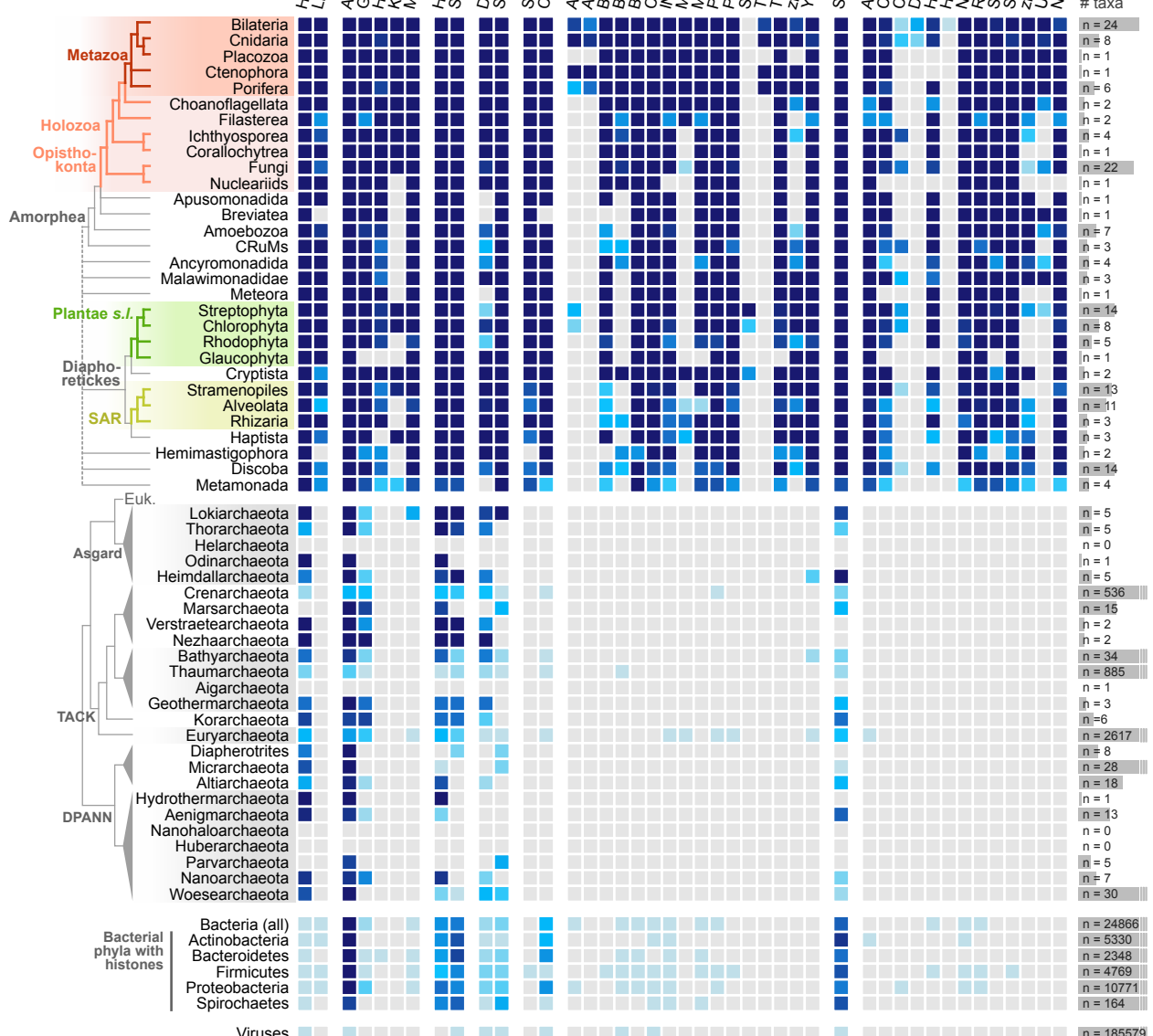

e

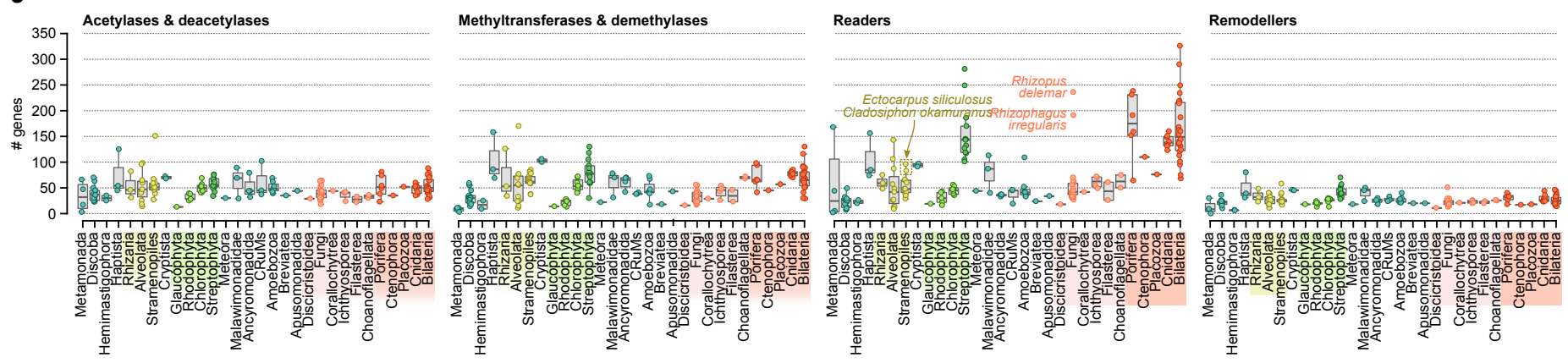


Figure 3. Taxonomic distribution of chromatin-associated gene classes. a, Summary of the seven classes of genes with chromatin-related activity covered in our survey: histone-specific hPTM writers (acetylases and methyltransferases), erasers (deacetylases and demethylases), readers, remodellers, and chaperones. b, Percentage of surveyed taxa containing homologs from each chromatin-associated gene class, for eukaryotes (top), archaea, bacteria, and viruses (bottom). Species-level tables are available in Supplementary Fig. 2. c, Number of eukaryotic genes classified in each of the chromatinassociated modification enzymes, readers, remodellers, and chaperones. d, Overlap between the taxon-level phylogenetic distribution of histones and chromatin-associated domains in archaea and four bacterial phyla, measured using the Jaccard index. e, Number of genes encoding writer, eraser, reader and remodeller domains, per species. 
bioRxiv preprint doi: https://doi.org/10.1101/2021.11.30.470311; this version posted December 1, 2021. The copyright holder for this

preprint (which was not certified by peer review) is the author/funder, who has granted bioRxiv a license to display the preprint in

\section{Figure 4} perpetuity. It is made available under aCC-BY-NC 4.0 International license.
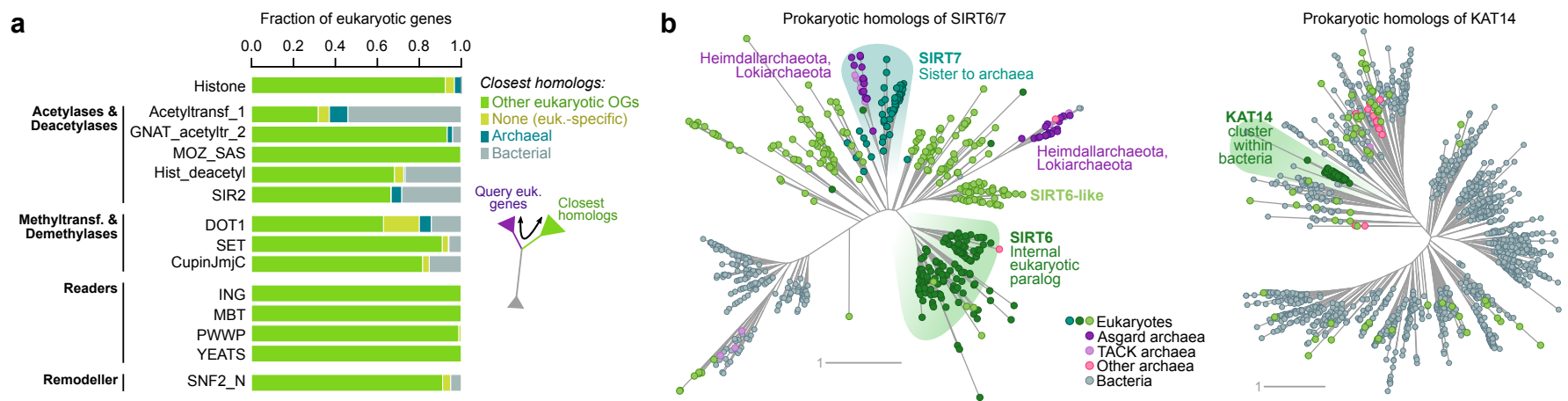

C
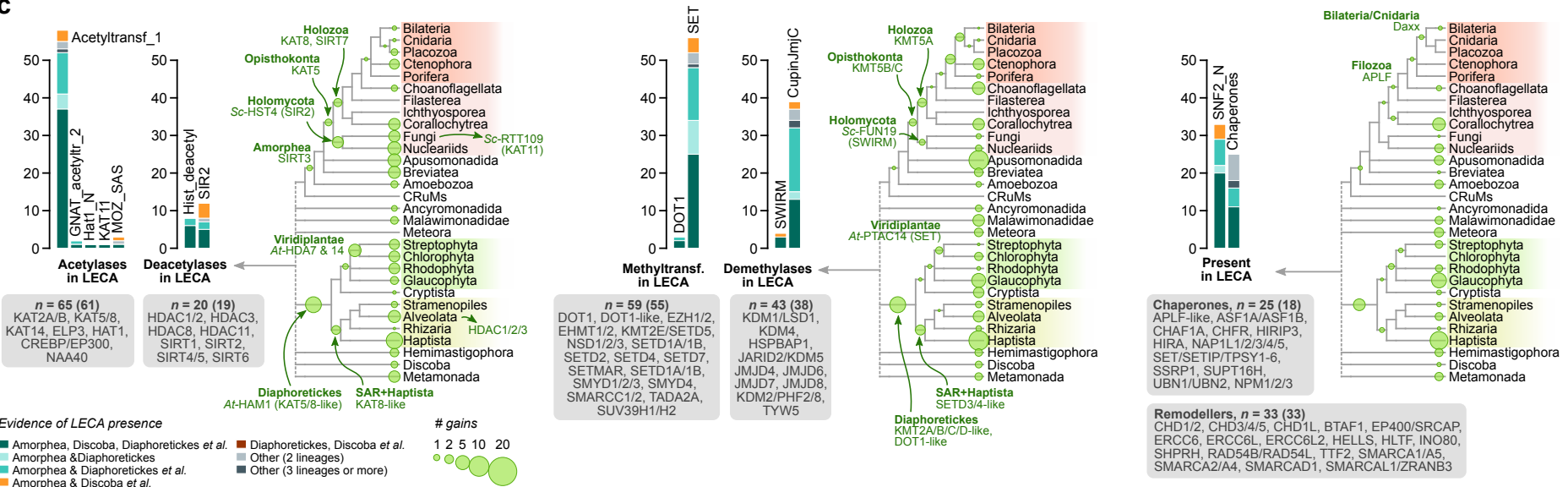

d Polycomb repressive
complex 2 (PRC2)

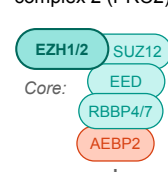

\section{PHF1/19 EZHIP* EPOP*
MMTF2}

Evolutionary origin:

O Metazoa O Holozoa O LECA
O Within Metazoa O Opisthokonta
Polycomb repressive complex 1 (PRC1)

ubiquitin
ligase

NG1A/B ancestral PCGF

non-canonical (2/4) non-canonical (3/5)

$\underbrace{}_{\text {YAF } 2 / R Y B P}$ PCGF $/ 4$ YAF2/RYBP UBEE2D3 WDR5/B

non-canonical (1) non-canonical (6)

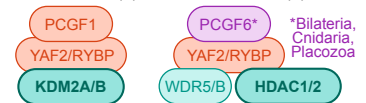

e

$$
\text { Suv }
$$

KMT1 (SUV39H1) KMT2 (Trithorax)

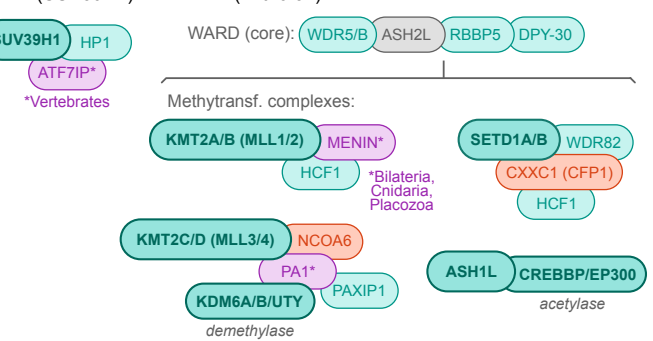

KMT3

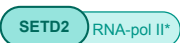

KMT4 (DOT1)

DOT1 TRRAP

(MLLT1/3 (ENL/AF9)

KMT5 (SUV4-20)

KMT5B/C
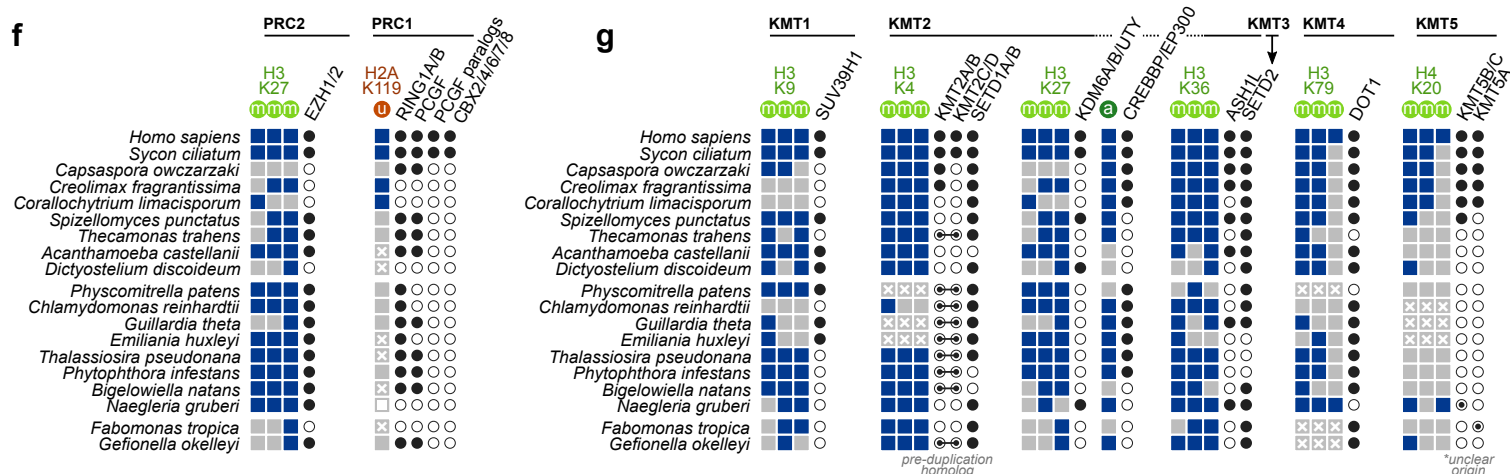

h

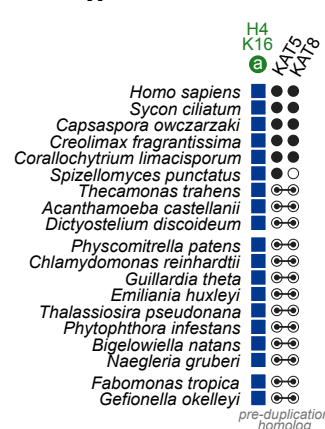


Figure 4. Origin and evolution of chromatin-associated gene families. a, Summary of phylogenetic affinities of the eukaryotic homologs of gene classes that are also present in prokaryotes. For each gene family, we evaluate whether it is phylogenetically closer to a majority ( $\geq 50 \%)$ of eukaryotic sequences from a different orthogroup (indicating intra-eukaryotic diversification), or to sequences from Bacteria or Archaea. b, Left, gene tree of eukaryotic and prokaryotic Sirtuin deacetylases, showcasing an example of a eukaryotic family that diversified within eukaryotes (SIRT6) and another one with close relatives in Asgard archaea (SIRT7). Right, gene tree of KAT14 acetylase, a eukaryotic orthogroup with bacterial origins. c, Evolutionary reconstruction of hPTM writer and eraser gene families, remodellers, and histone chaperones along the eukaryotic phylogeny, including the number of genes present in the last eukaryotic common ancestor (LECA). Barplots indicate the number of orthologs of each gene family present at the LECA (at 90\% posterior probability; see Methods) and whether the presence of a given orthogroup at LECA is supported by its conservation in various earlybranching eukaryotic lineages (Amorphea, Discoba, Diaphoretickes and others). The list of ancestral gene families below each plot is non-exhaustive. Two ancestral gene counts are provided: all families at presence probability above $90 \%$, and the subset of these that is present in at least two of the main eukaryotic early-branching lineages (Amorphea, Diaphoretickes, and Discoba). Source data in Table S5. d-e, Evolutionary origin of the components of the Polycomb repressive complexes (PRC2 and PRC1) and Trithorax-group proteins (KMT1 to 5). $\mathbf{f}-\mathbf{h}$, Side-by-side comparison of the presence of individual hPTM marks and various subunits of the Polycomb and Trithorax complexes, as well as other hPTM writers, responsible for their deposition. 
bioRxiv preprint doi: https://doi.org/10.1101/2021.11.30.470311; this version posted December 1, 2021. The copyright holder for this

preprint (which was not certified by peer review) is the author/funder, who has granted bioRxiv a license to display the preprint in

Figure 5 perpetuity. It is made available under aCC-BY-NC 4.0 International license.

a

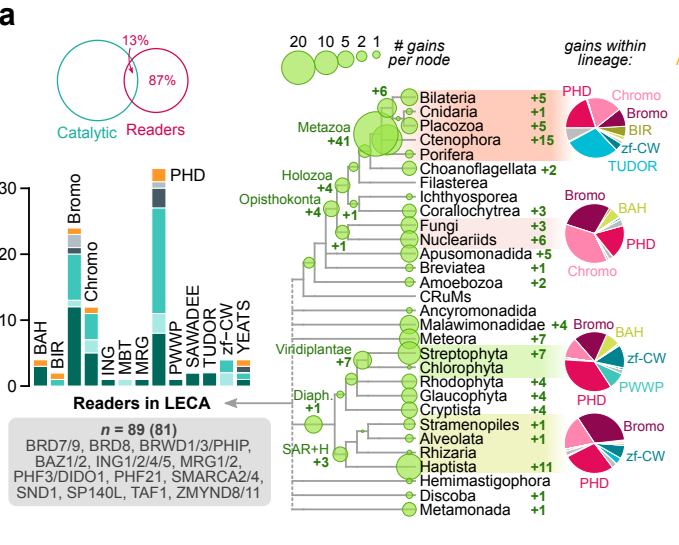

b

Homo sapiens

Readers (97) Catalytic (96)
Metazoa
LECA

Drosophila melanogaster
Readers (80) Catalytic (81)

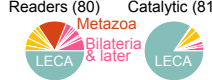

Nematostella vectensis
Readers (101) Catalytic (105)

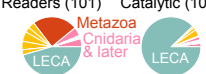

Capsaspora owczarzaki

Readers (57) Catalytic (95)

$\bigcup_{\text {LECA }}^{\substack{\text { Species- } \\ \text { Specitic }}}$

Arabidopsis thalian

Readers (57) Catalytic (93)

(LECA)

C

Domain co-occurrence with Chromo

Domain co-occurrence with PHD

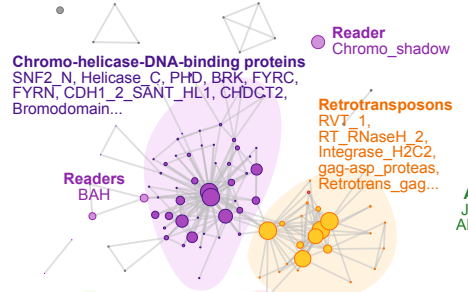

DNA transposons
DDE_1. DDE TITp 4 -
Dimer_Tnp_hAT

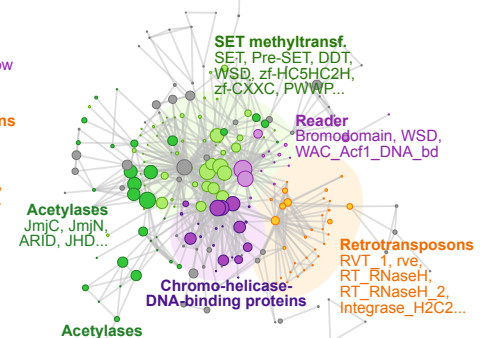

SET methyltransf.
SET, Pre-SET.

- Reader

Acetyláses

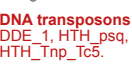

\#cc-occurrences
with core domain

10 2050100

d
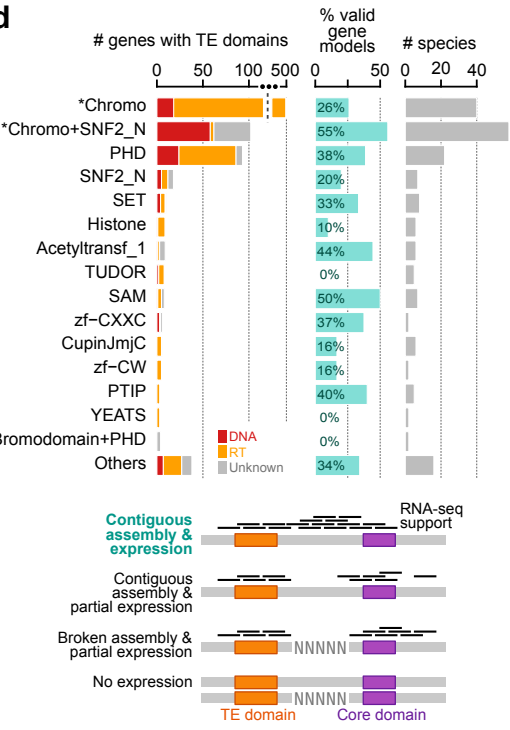

f

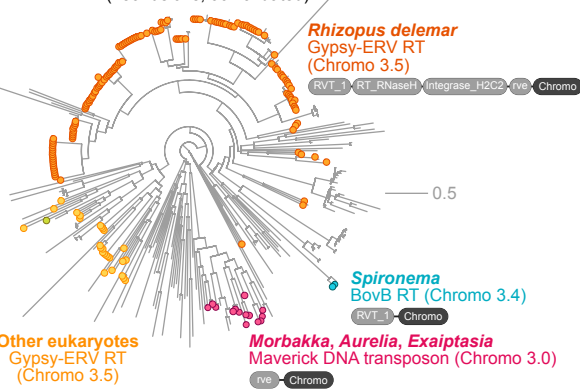

e

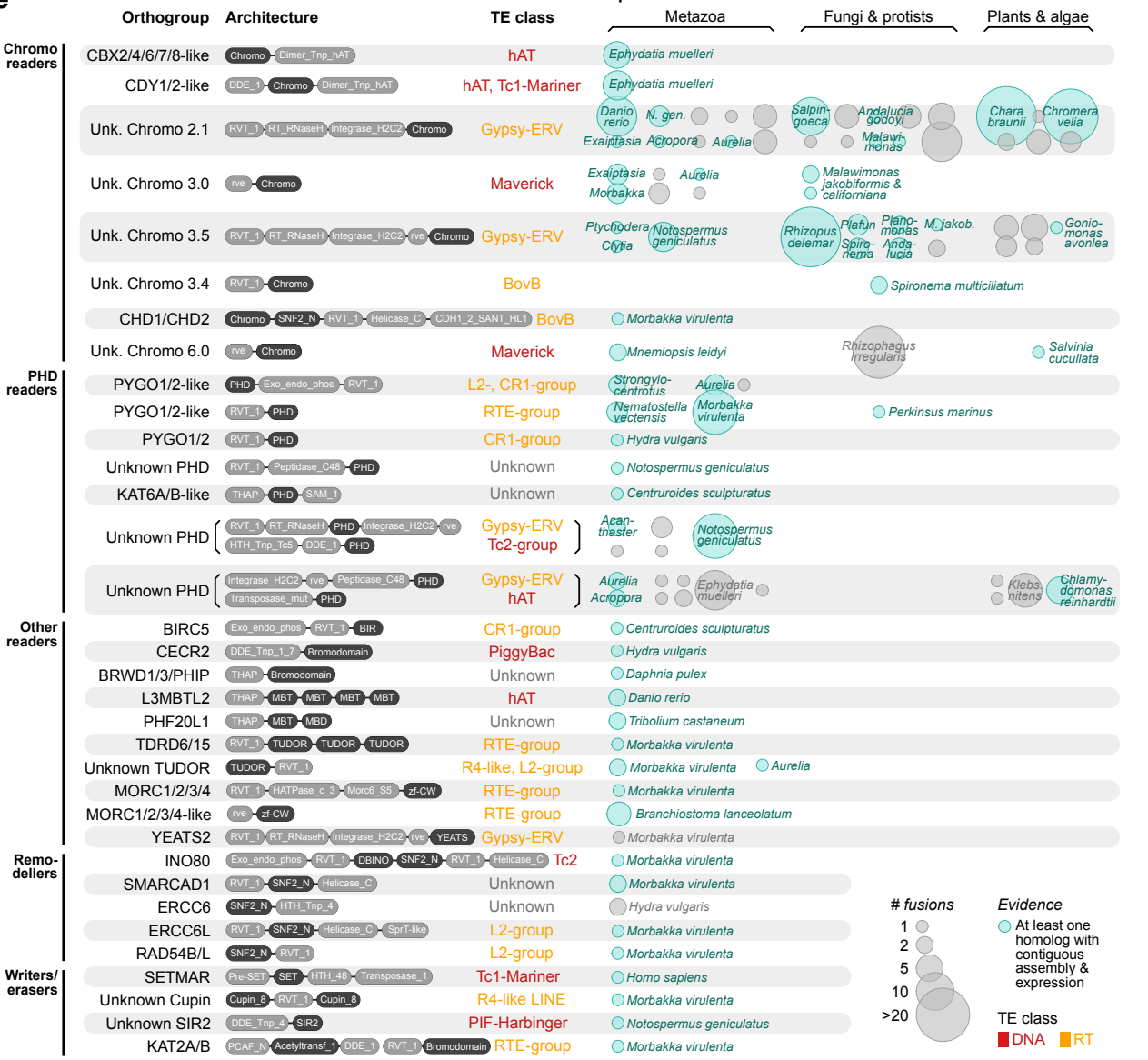

g

Hist- $\begin{aligned} & \text { Acetylases, } \\ & \text { ones } \\ & \text { Deacetylases }\end{aligned}$
Remodeller

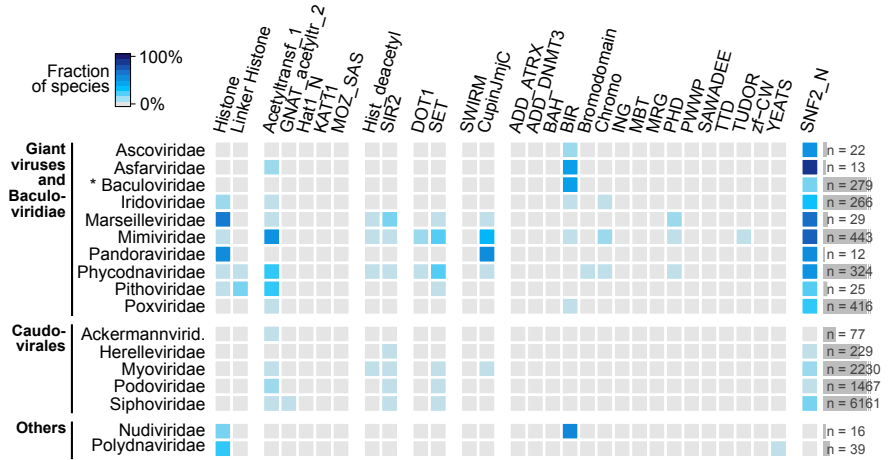

h

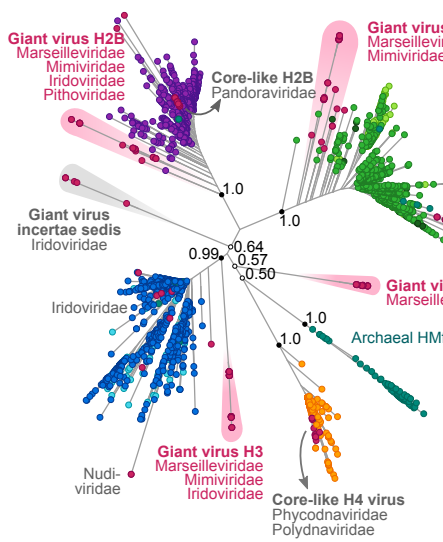

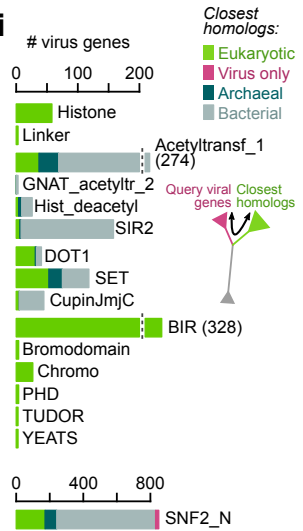


Figure 5. Evolution of chromatin readers and capture of chromatin proteins by transposable elements and viruses. a, Evolutionary reconstruction of reader gene families along the eukaryotic phylogeny, highlighting the number of gains along the eukaryotic phylogeny (at $90 \%$ posterior probability). The Euler diagram at the top shows the overlap between presence of chromatin-associated catalytic domains and readers. The barplot at the left indicates the number of orthologs of each gene family present at the LECA and whether their presence is supported by its conservation in various early-branching eukaryotic lineages (Amorphea, Discoba, Diaphoretickes, and others). Pie plots at the right summarize the number of orhogroups from each gene family gained within selected lineages: Metazoa, Holomycota, Viridiplantae and SAR+Haptophyta. b, Number of reader or catalytic orthogroups gained at each node in the species tree, for selected species. Source data in Table S5. c, Networks of protein domain co-occurrence for Chromo and PHD readers. Each node represents a protein domain that co-occurs with Chromo or PHD domains, and node size denotes the number of co-occurrences with either Chromo or PHD. Edges represent co-occurrences between domains. Groups of frequently co-occurring protein domains have been manually annotated and colour-coded, which has revealed sub-sets of retrotransposon and DNA transposon-associated domains. d, Number of chromatin-related eukaryotic genes fused with transposons grouped by gene family (left), including the fraction which are classified as valid gene models based on expression and assembly data (centre); and the number of species where each type of fusion is found (right). The number of fusion events are coloured according to their similarity with known DNA transposons (red) or retrotransposons (orange) from the Dfam database (see Methods). (*) The 'Chromo' category excludes genes containing other chromatin-associated protein domains such as SNF2_N (listed separately as 'Chromo+SNF2_N', which includes remodellers with the domain of unknown function DUF1087, which is also common in DNA transposons). e, Selected examples of transposon fusion domains classified by orthogroup, including their archetypical protein domain architecture, homology to transposon class, their phylogenetic distribution, and number of fusion genes. Only orthogroups with at least one valid gene model are listed. Complete list in Supplementary Fig. 5 and source data available in Supplementary Table 6. f, Example tree of Chromo readers, highlighting genes with fused TEassociated domains and their consensus domain architectures. g, Fraction of viral genomes containing homologs from each chromatin gene family, for nucleocytoplasmic giant DNA virus families (top) and other taxa containing histone domains (Nudiviridae, Polydnaviridae; bottom). h, Phylogenetic analysis of histone domains, with a focus on viral homologs. Statistical supports (approximate Bayes posterior probabilities) are shown for the deepest node of each canonical eukaryotic or archaeal histone clade. The inset table summarizes the presence of doublet histone genes per linage. i, Number of viral homologs in each chromatin-associated gene family, classified according to their closest cellular homologs (eukaryotes, bacteria or archaea) in phylogenetic analyses (see Methods). Source data available in Supplementary Table 6. 
bioRxiv preprint doi: https://doi.org/10.1101/2021.11.30.470311; this version posted December 1, 2021. The copyright holder for this

preprint (which was not certified by peer review) is the author/funder, who has granted bioRxiv a license to display the preprint in

\section{Figure 6} perpetuity. It is made available under aCC-BY-NC 4.0 International license.

Pre-LECA

- Nucleosomes: no tails

- No canonical histones

- Catalytic structural

domains
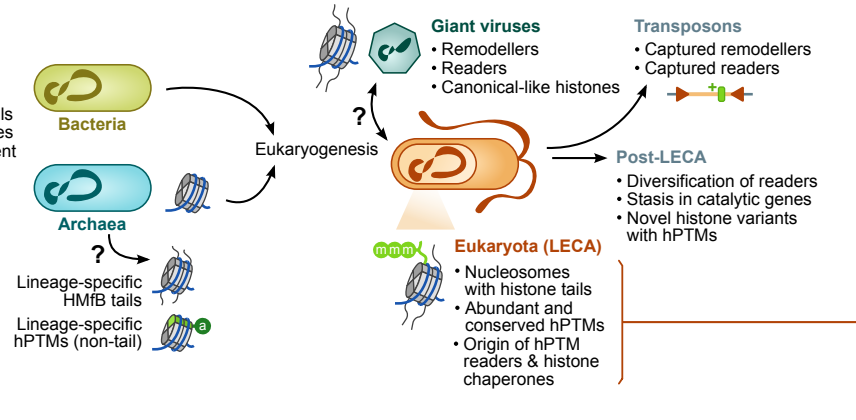

b
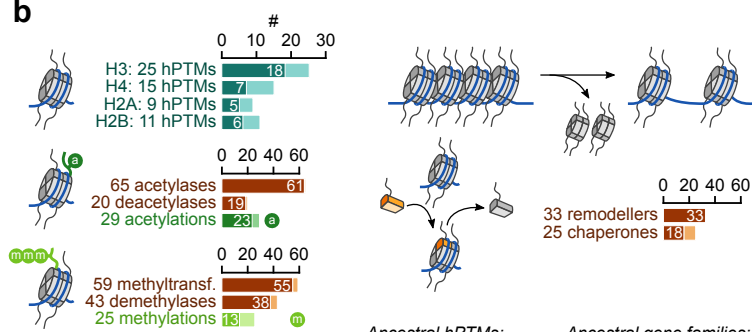

33 remodellers $\begin{array}{r}044060 \\ \hline \\ \hline\end{array}$

Ancestral hPTMs: $\quad$ Ancestral gene families:

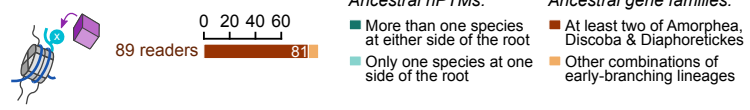

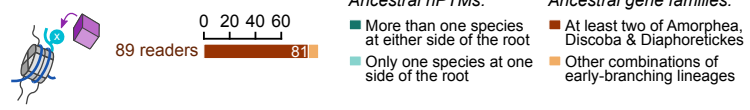

c
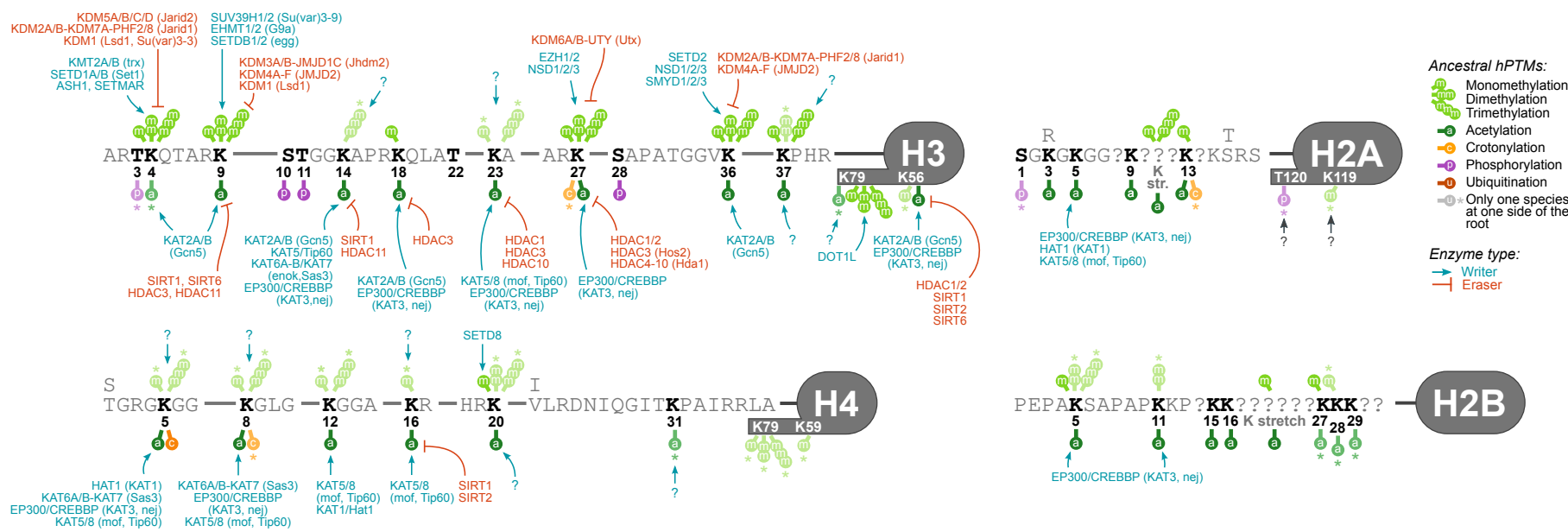
Figure 6. Chromatin evolution and eukaryogenesis. a, Summary of events in chromatin evolution prior to, during and after the origin of eukaryotes. $\mathbf{b}$, Number of chromatin-related gene families and hPTM marks inferred to have been present at the LECA. Ancestral gene counts are indicated at $>90 \%$ probability. For gene counts, numbers within bars indicate the subset of families present in at least two of the most deeply-sampled early-branching eukaryotic lineages (Amoropha, Diaphoretickes, and Discoba). For hPTMs, the ancestral counts have been inferred using Dollo parsimony assuming a Diaphoratickes - Amorphea split at the root of eukaryotes, and numbers within bars indicate the number of hPTMs whose ancestral presence is supported by more than one species at both sides of the root. c, hPTMs inferred to be present in the last eukaryotic common ancestor (LECA) based on Dollo parsimony. Only amino-acid positions conserved in all eukaryotes in our dataset are shown. Asterisks indicate modifications whose presence at the LECA is supported by just one species at either side of the root. The inferred LECA presence of known writing/erasing enzymes associated to these hPTM is indicated. 
bioRxiv preprint doi: https://doi.org/10.1101/2021.11.30.470311; this version posted December 1, 2021. The copyright holder for this

preprint (which was not certified by peer review) is the author/funder, who has granted bioRxiv a license to display the preprint in

\section{Supplementary Figure S1} perpetuity. It is made available under aCC-BY-NC 4.0 International license.

a) Histone classification
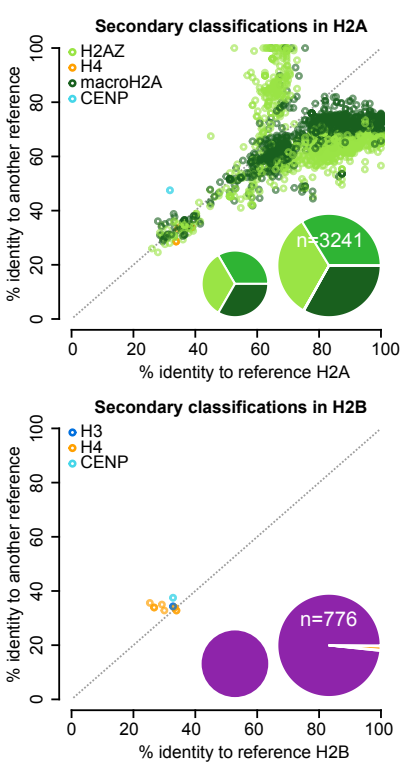
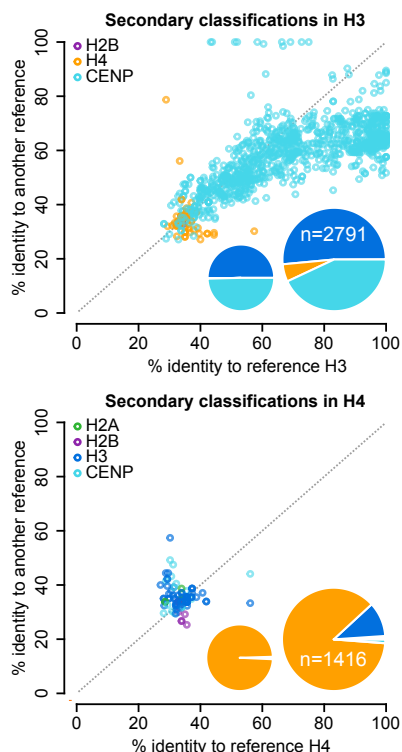

b) Proteomics evidence summary

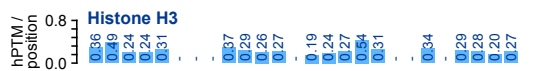

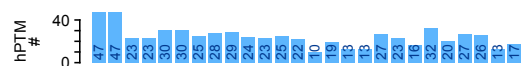

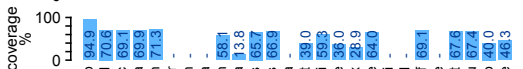

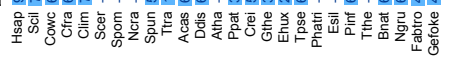

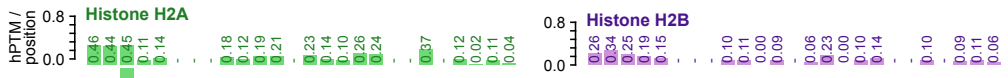

质 $\begin{array}{r}40 \\ 0\end{array}$

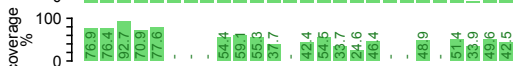

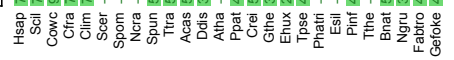

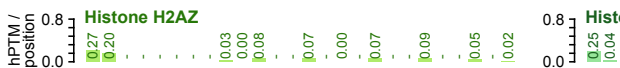

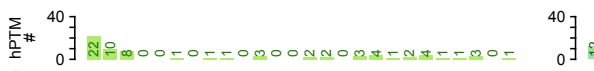

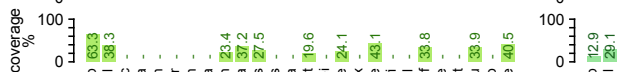

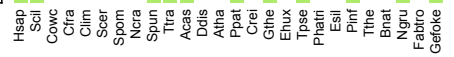

8 Histone $\mathrm{H} 4$

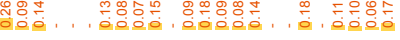

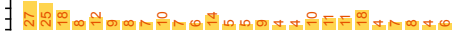

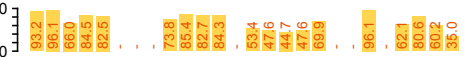

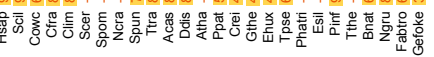
${ }_{0}^{40} \exists$ 国

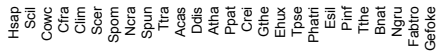

listone macroH2A

으른

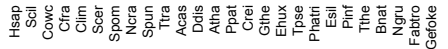

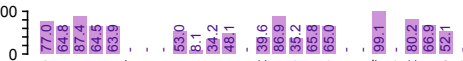

c) hPTMs in $\mathrm{H} 2 \mathrm{AZ}$, macroH2A and $\mathrm{H} 1$
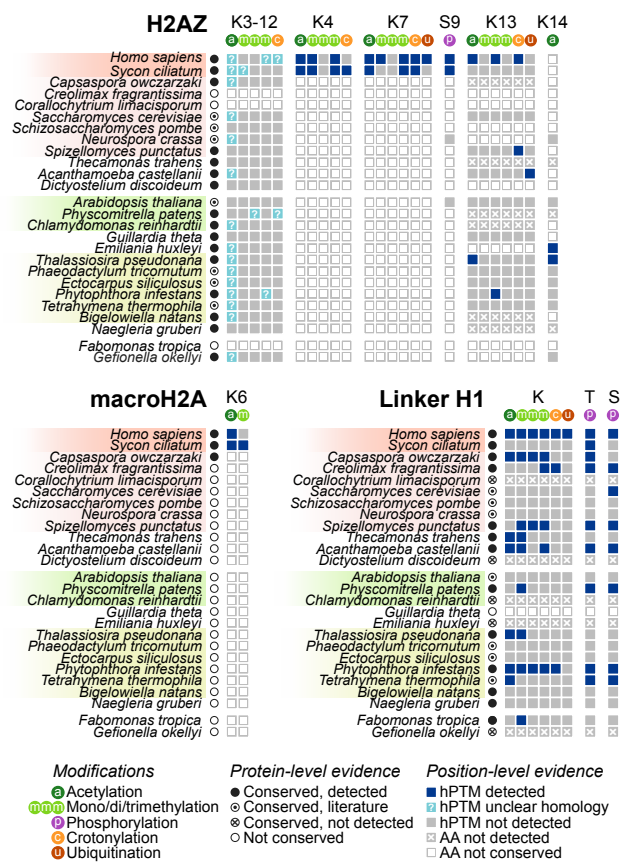

d) Archaeal histone tails
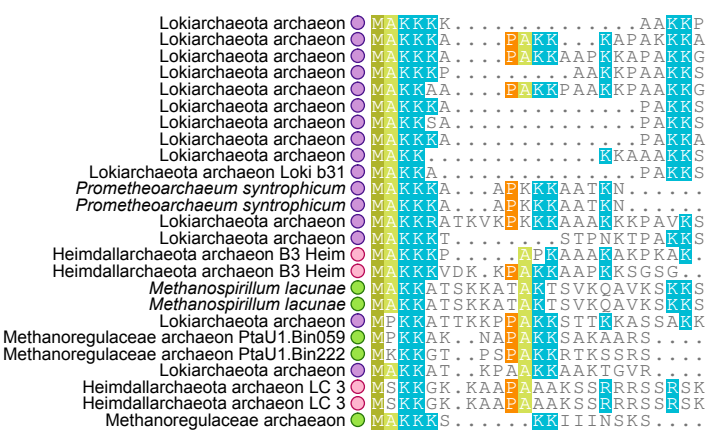

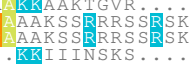
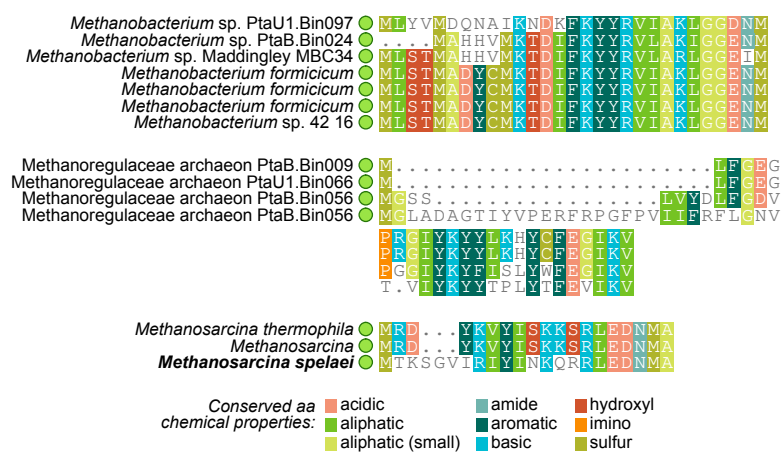

e) Histone pairs in eukaryotes

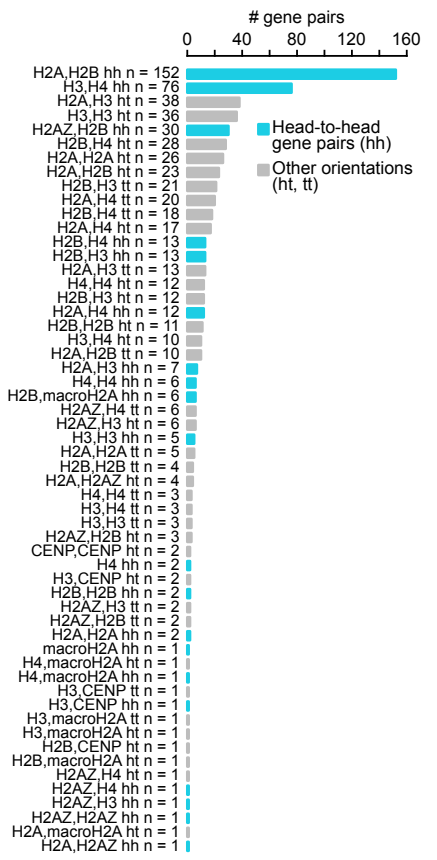

f) Histone pairs per species

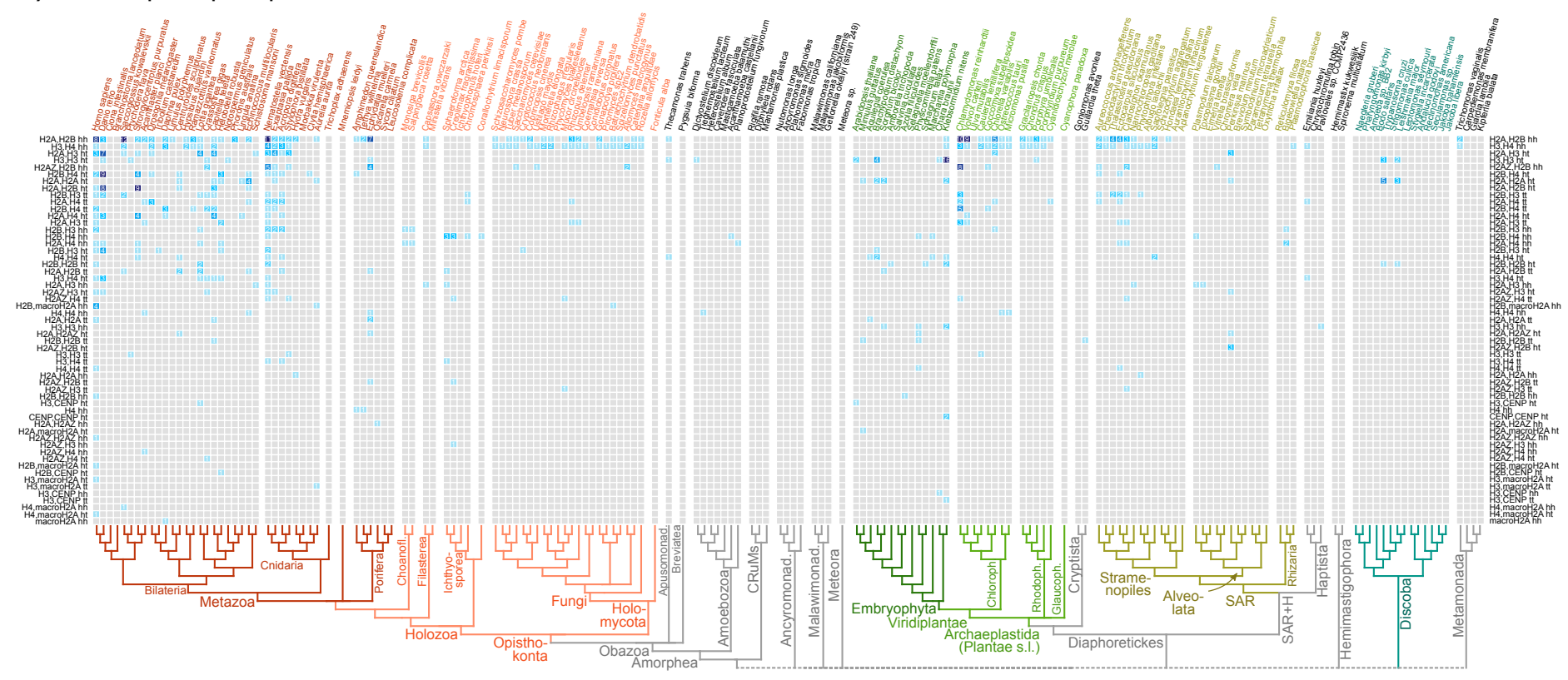


Supplementary Fig. 1. Histone classification and evolution. a, Primary and secondary alignments of histone-fold containing proteins classified as canonical H2A, H2B, H3 and H4, based on identity to reference sequences in HistoneDB. Pie plots represent the number of alignments to HistoneDBannotated sequences, for the entire dataset (prokaryotic, eukaryotic and viral sequences, large pie plots in the inset) and the eukaryotic subset (smaller plots in the inset). For those proteins that align to more than one canonical histone or major variant (macroH2A, H2AZ or CENP), the scatter plots represent the relative identity between the primary (horizontal axis) and secondary alignment(s) (vertical axis). b, Proteomics detection coverage (\% of amino acids), number of hPTMs and number of hPTMs per covered position, for the best-covered histone in each species in our proteomics survey. c, Evidence of hPTM conservation in the major histone variants $\mathrm{H} 2 \mathrm{AZ}$ and macroH2A (conserved positions only), as well as any position in the linker histones H1. d, Alignments of putatively conserved histone $\mathrm{N}$-tails in archaea. Conserved amino-acids are colour-coded according to chemical properties. Dots next to species names are colour-coded according to taxonomy (same as Fig. 2c). e, Aggregated counts of histone gene pairs, classified according to histone type and orientation. f, Number of collinear pairs of histone-encoding genes per species, classified according to their histone types and relative orientation (head-to-head, hh; head-to-tail, ht; and tail-to-tail, tt). Source data available in Table S2. 
bioRxiv preprint doi: https://doi.org/10.1101/2021.11.30.470311; this version posted December 1, 2021. The copyright holder for this preprint (which was not certified by peer review) is the author/funder, who has granted bioRxiv a license to display the preprint in

\section{Supplementary Figure S2} perpetuity. It is made available under aCC-BY-NC 4.0 International license.

a) Gene family presence in Archaea phyla

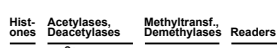
$\stackrel{\substack{\text { Remodoller } \\ \perp \text { Histone chaperones }}}{4}$

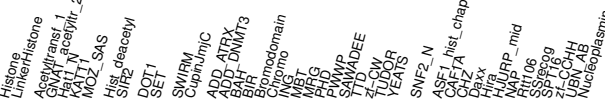
最最回哭㽞

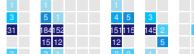

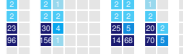

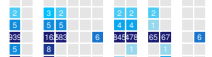

兽要

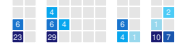

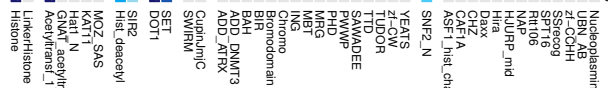

b) Gene family presence in Bacteria phyla

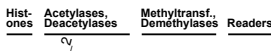

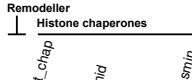

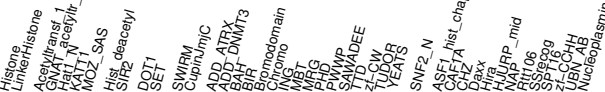

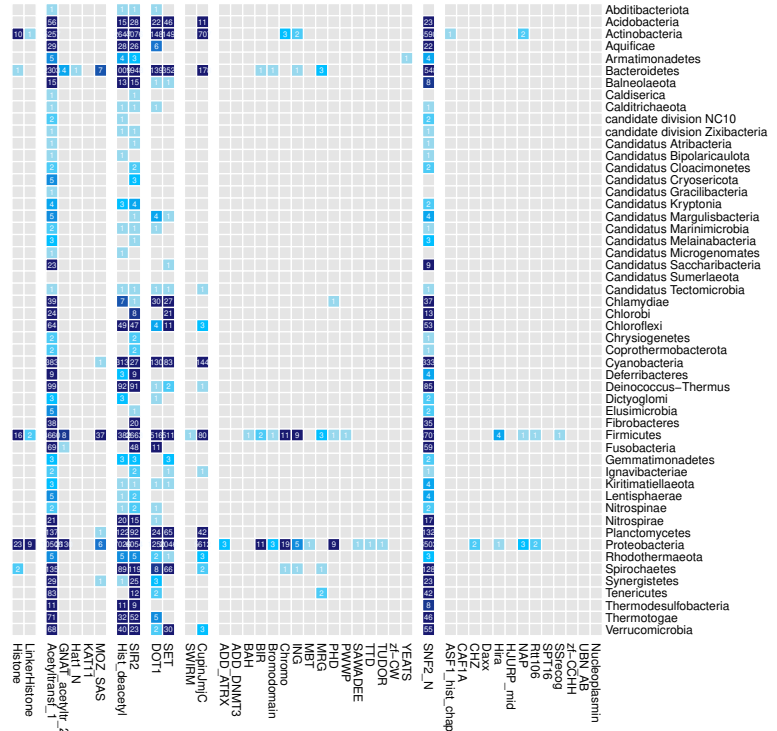

c) Gene family presence in Virus families

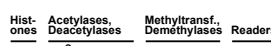

$\stackrel{\substack{\text { Remodoller } \\ \perp} \text { Histone chaperones }}{ }$

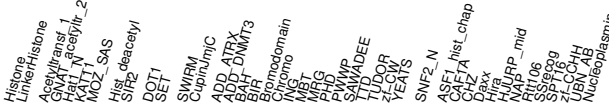
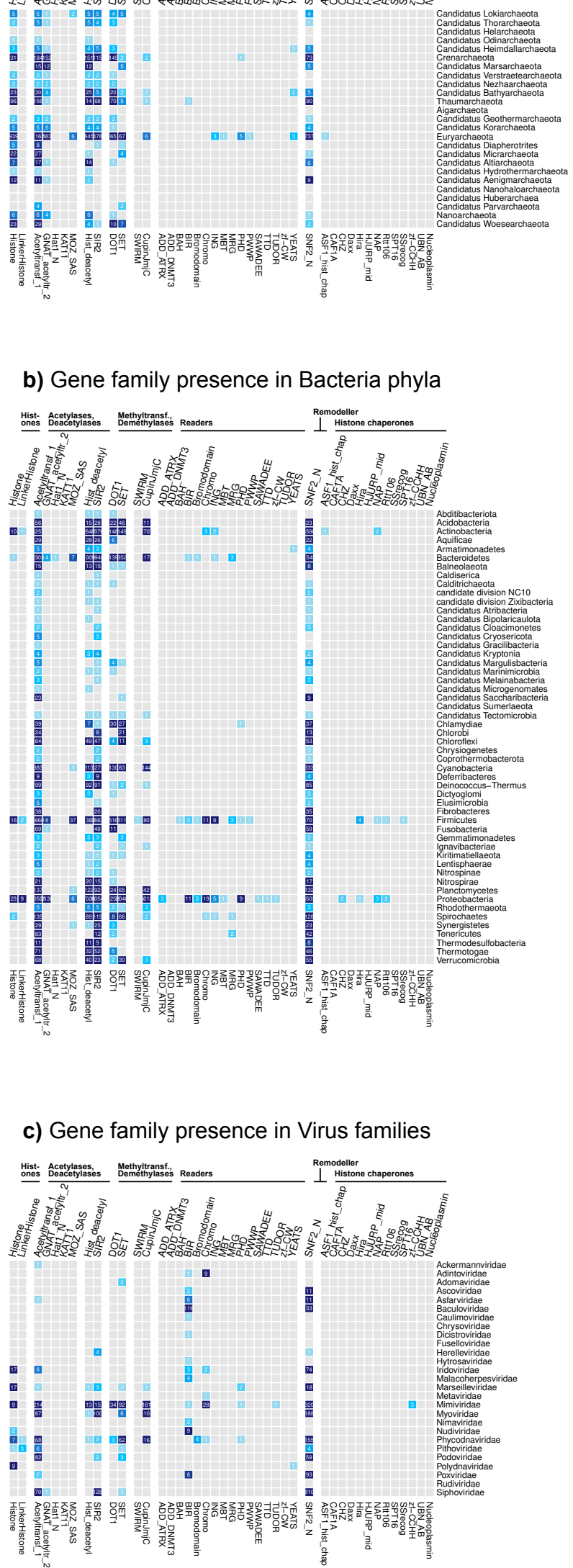
西 d) Gene family counts in eukaryotes

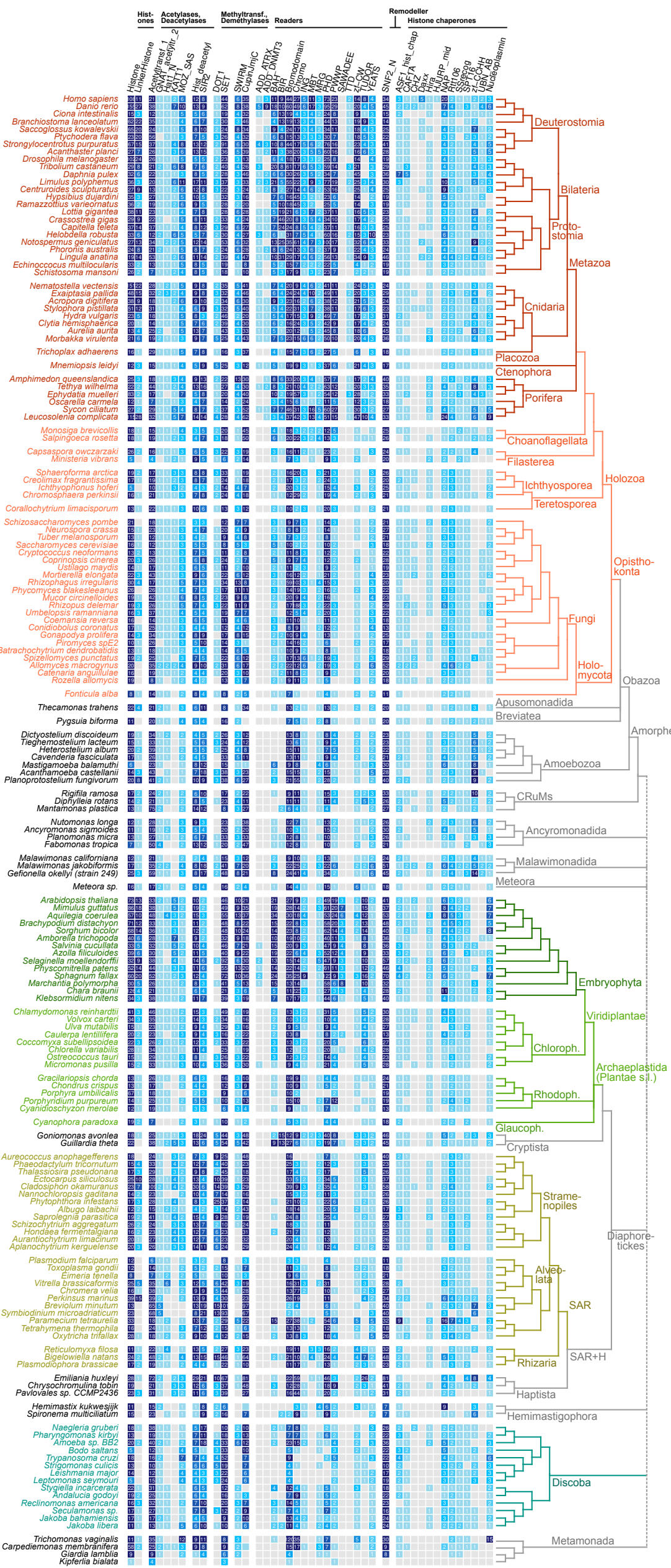


Supplementary Fig. 2. Gene family counts. a-c, Number of taxa within each lineage that contain chromatin-associated genes, for archaeal, bacterial (per phyla) or viral (per family) genomes. d, Number of genes encoding core domains that define chromatin-associated gene families per eukaryotic genome/transcriptome. 
bioRxiv preprint doi: https://doi.org/10.1101/2021.11.30.470311; this version posted December 1, 2021. The copyright holder for this preprint (which was not certified by peer review) is the author/funder, who has granted bioRxiv a license to display the preprint in Supplementary Figure S3 perpetuity. It is made available under aCC-BY-NC 4.0 International license.

a) Rates of orthogroups gain/loss

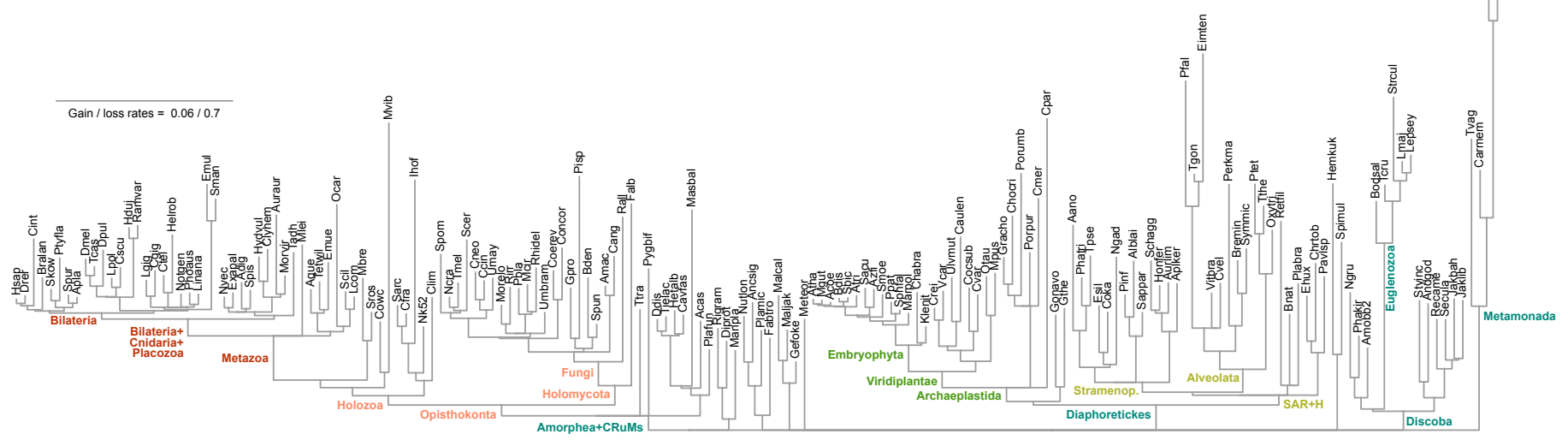

b) Domain architecture conservation
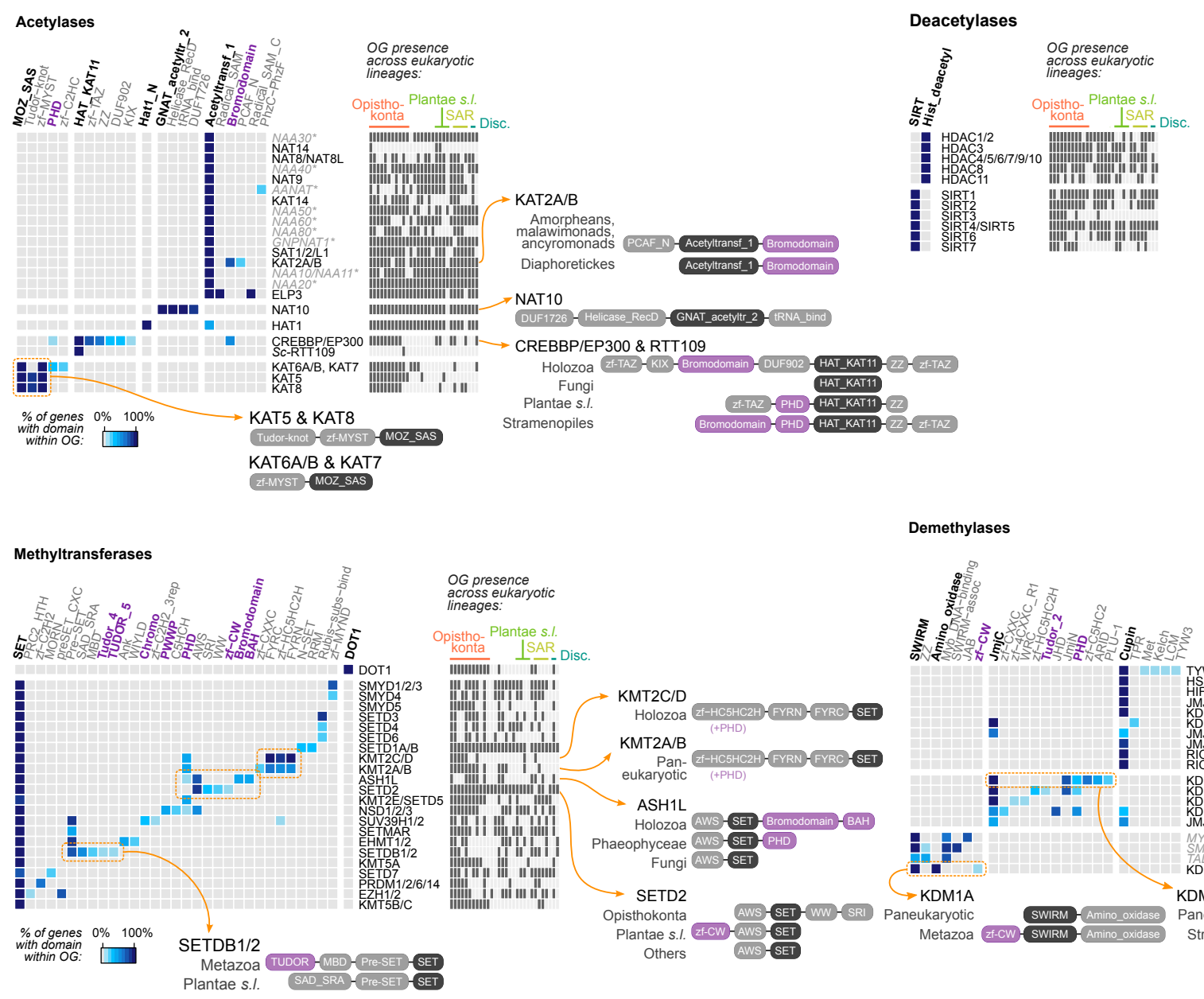

Demethylases
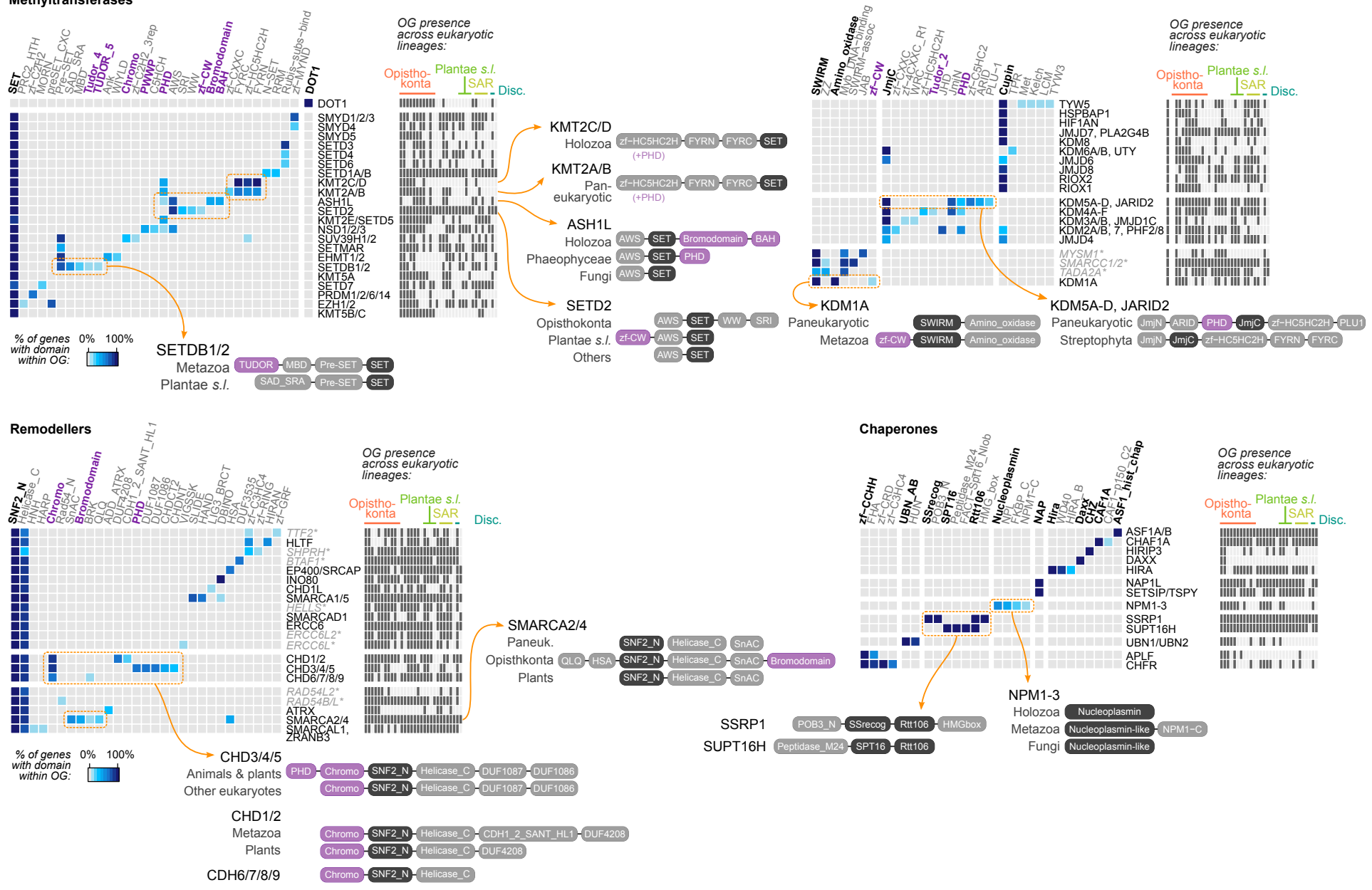

OG presence
across eukaryotic
lineages: Dpistho- Plantae s.l.
konta ISAR Dis

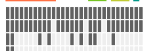
||

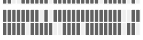
네II I'III II I I

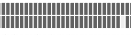
IIIII I II II IIII' I IIII I I I । 
Supplementary Fig. 3. Evolutionary reconstruction and domain architecture conservation. a, Species tree of eukaryotes used in the ancestral reconstruction analysis, with branch lengths calibrated to the gain/loss rates of Pfam domains (see Methods). Available in Table S1. b, Conservation of archetypical protein domain architectures across orthogroups, in acetylases, deacetylases, methyltransferases, demethylases, remodellers and chaperones. In each heatmap, we indicate the fraction of genes within an orthogroup (rows) that contain a specific protein domain (columns). Domains in bold are catalytic (black) or reader (purple) functions. At the right of each heatmap, we summarize the presence/absence profile of each orthogroup across eukaryotic lineages (as listed in Fig. 1a). 
bioRxiv preprint doi: https://doi.org/10.1101/2021.11.30.470311; this version posted December 1, 2021. The copyright holder for this

preprint (which was not certified by peer review) is the author/funder, who has granted bioRxiv a license to display the preprint in

Supplementary Figure S4 perpetuity. It is made available under aCC-BY-NC 4.0 International license.

a) Overlap between readers \& catalytic genes

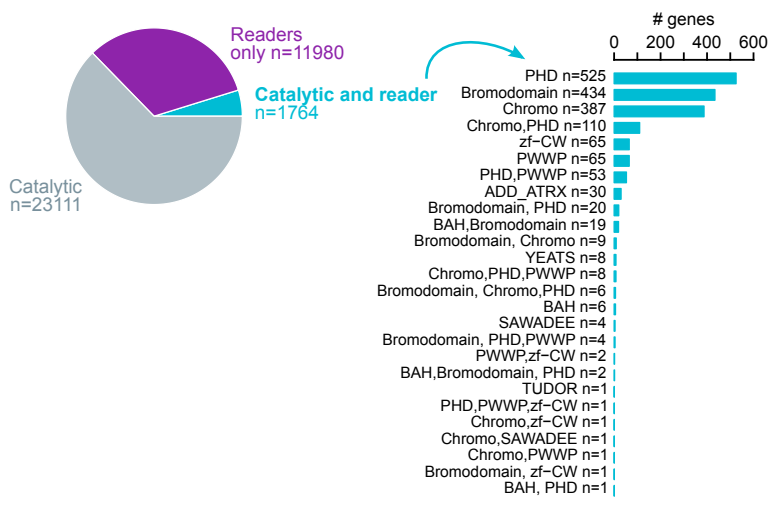

b) Domain redundancy in readers

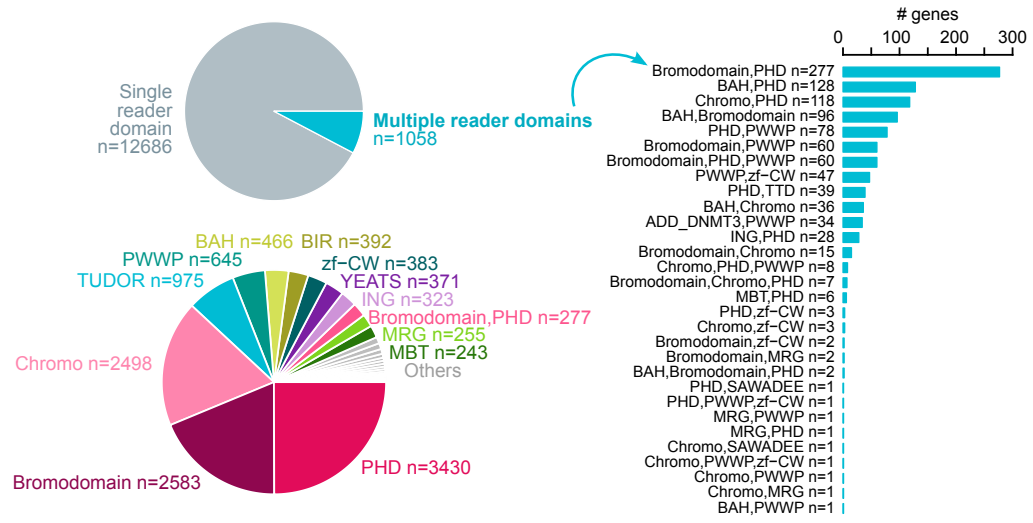

c) Gains of reader families
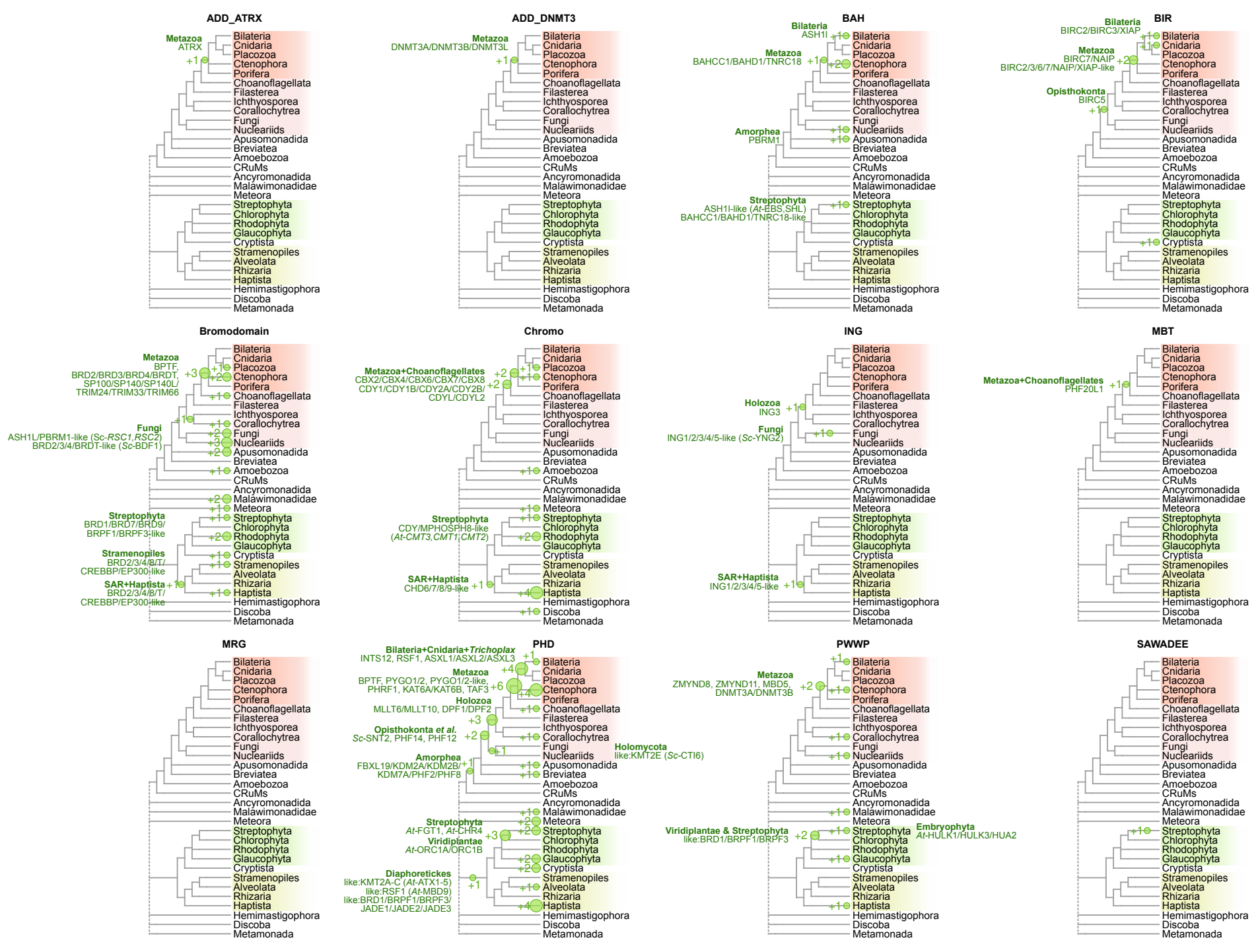

Viridiplantae \& Streptorhyta
like:BRD1/BRPF1/BRPPF3 +2

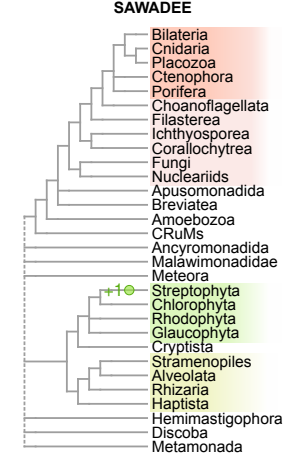

TTD
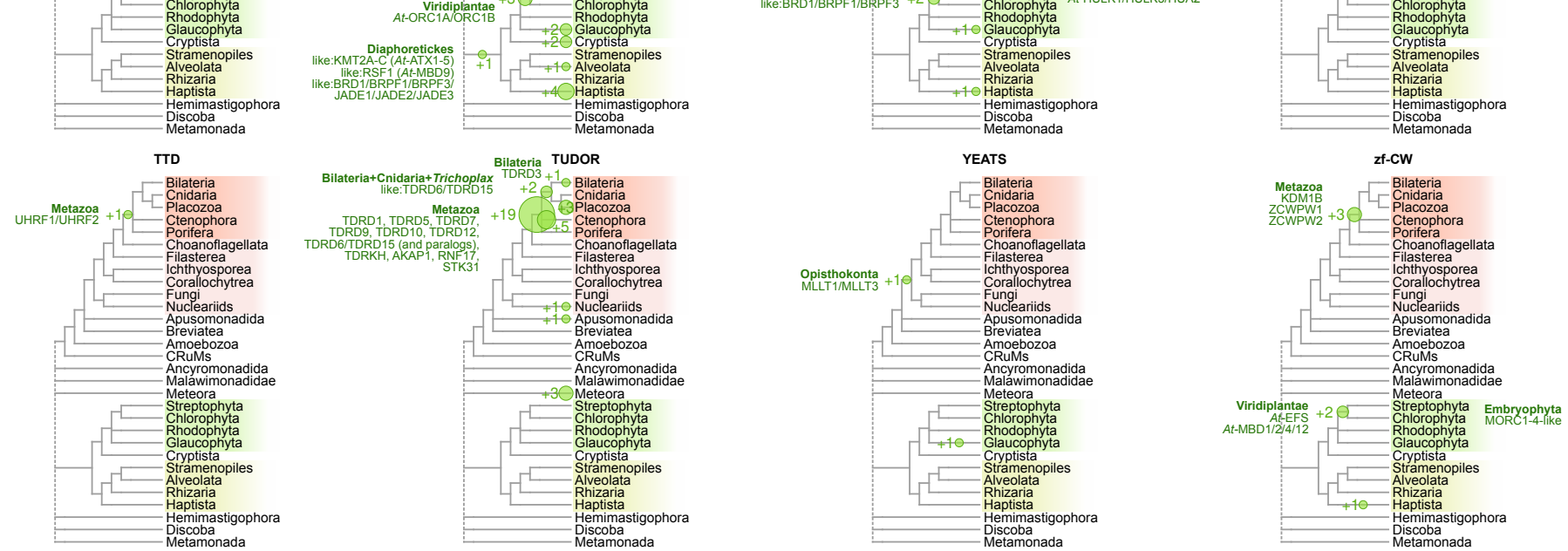
Supplementary Fig. 4. Evolution of the hPTM reader toolkit. a, Pie plot representing the number of genes classified as part of the catalytic (acetylases, deacetylases, methyltransferases, demethylases, remodellers or chaperones) or reader families, or as both. The barplot at the right shows the most common combinations of reader domains in genes classified with both reader and catalytic functions. b, Pie plots representing the number of reader domain-encoding genes classified according to whether they contain one type of reader domain (e.g., PHD) or more than one (e.g., PHD + PWWP; top), and the most common combinations (bottom). The barplot at the right shows the most common combinations of reader domains among genes with multiple reader domains. c, Summary of gene family gains per reader family, with example cases highlighted in selected nodes. Node size is proportional to number of gains at $90 \%$ probability. 
bioRxiv preprint doi: https://doi.org/10.1101/2021.11.30.470311; this version posted December 1, 2021. The copyright holder for this

preprint (which was not certified by peer review) is the author/funder, who has granted bioRxiv a license to display the preprint in

Supplementary Figure S5

a) Validation

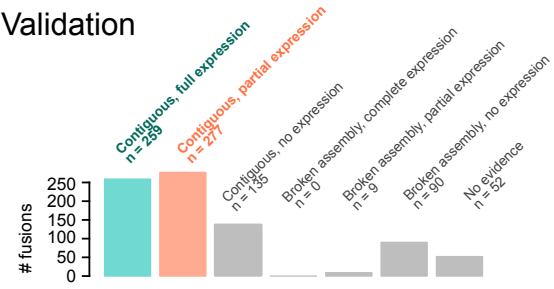

b) Summary of TE fusions

Histone

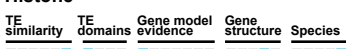

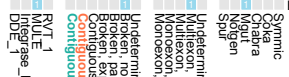

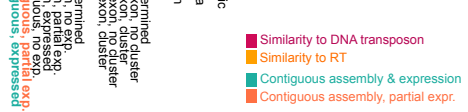

Acetyltransf_1

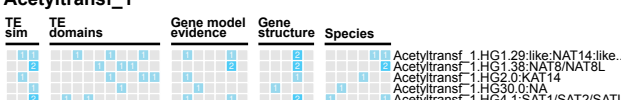

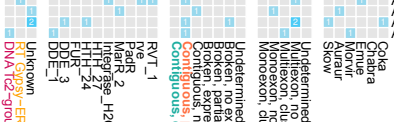

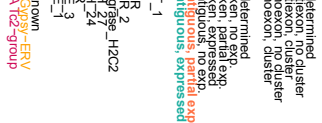

CupinJmjC

similarity $\begin{gathered}\text { TE } \\ \text { domains }\end{gathered}$

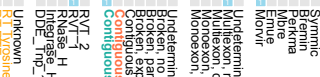

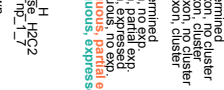

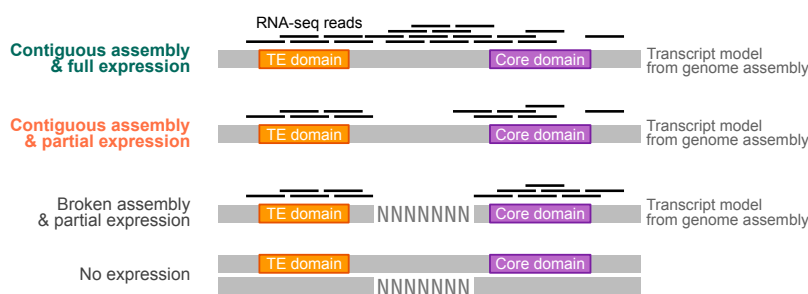

SIR2

TE TE Gene model Gene
sim dom evidence
structure Species

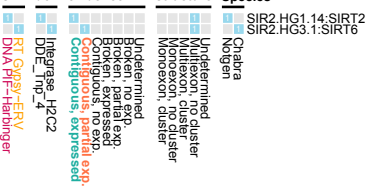

SWIRM

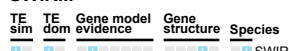
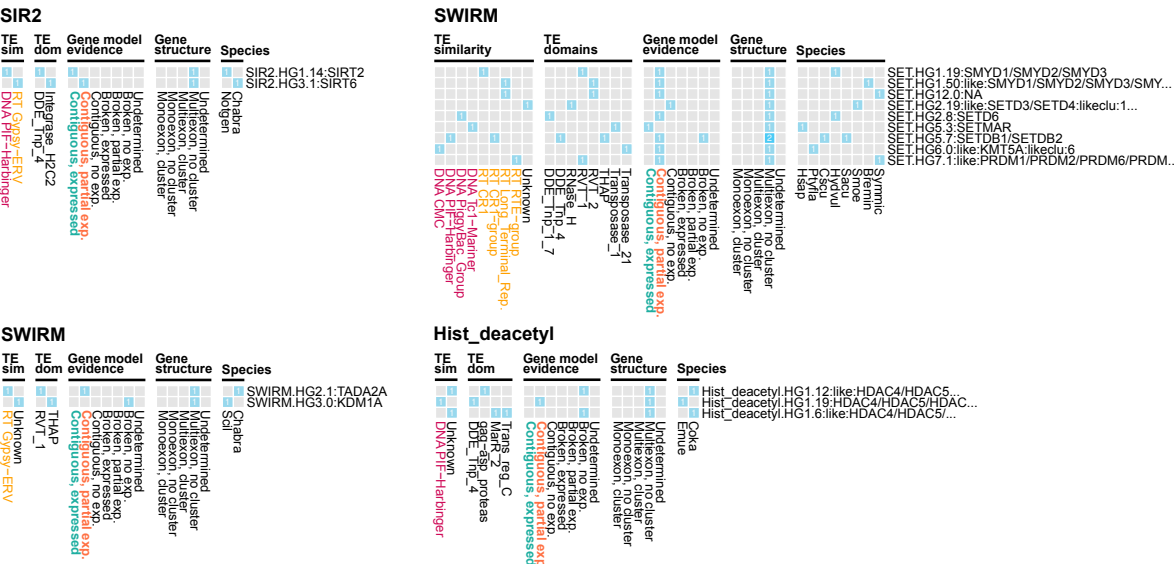

Hist_deacetyl

SEm TEm

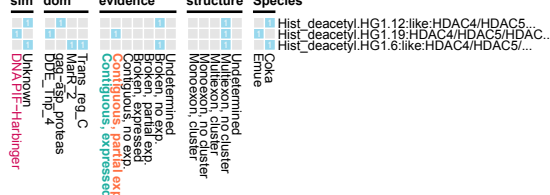

BIR

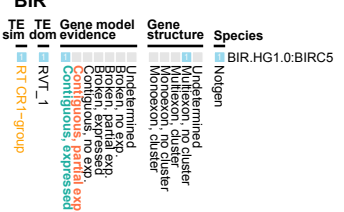

Chromo

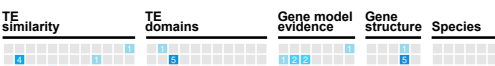

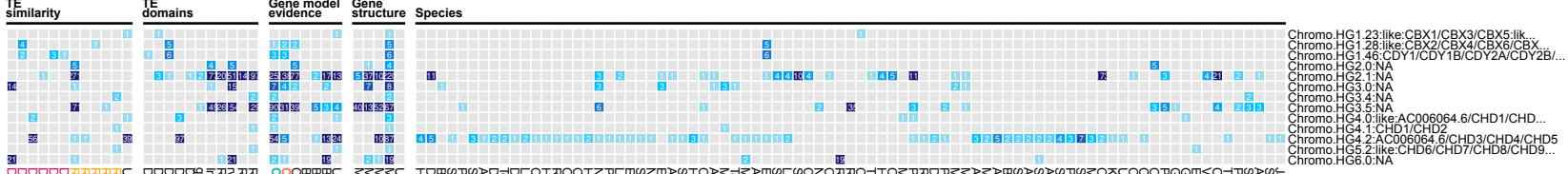

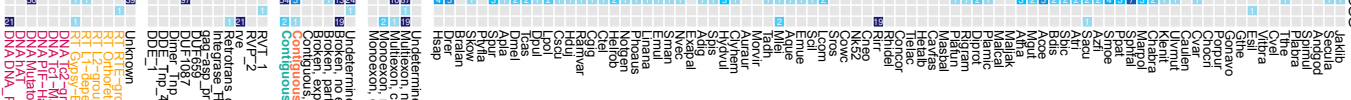

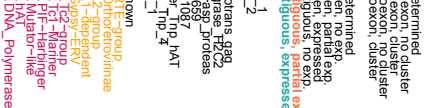

PHD

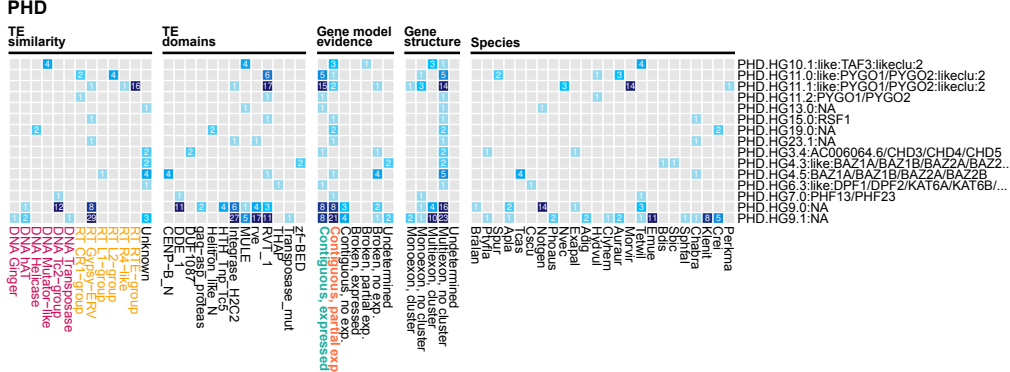

SWIRM

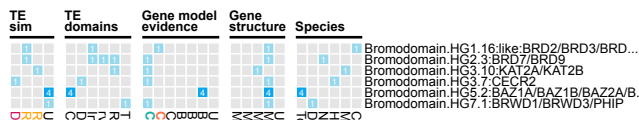

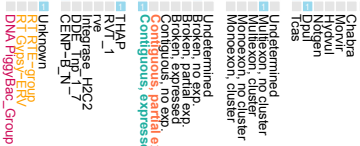
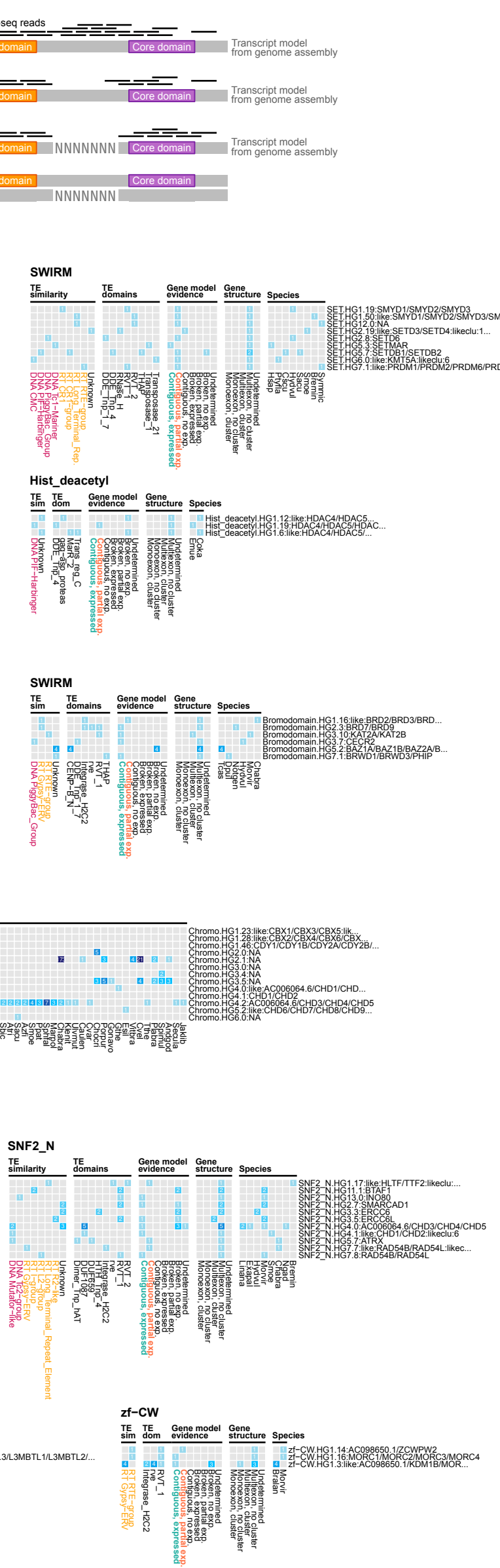

TUDOR

TE
Similarity
TE
domains

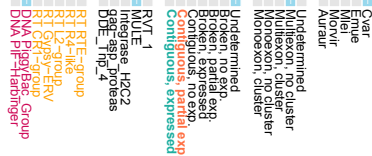

MBT

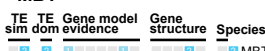

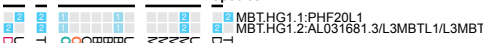

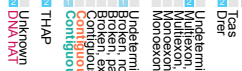

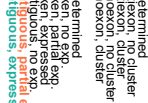

MRG

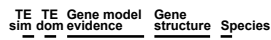

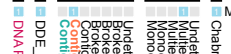

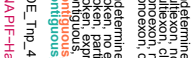

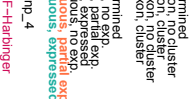

YEATS TES

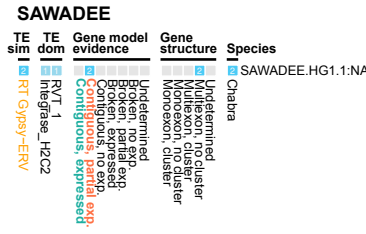

IE TE $\begin{aligned} & \text { Gene model } \\ & \text { sim done } \\ & \text { evidence }\end{aligned}$ structure Species

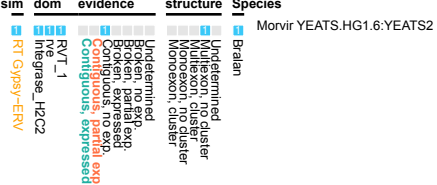


Supplementary Fig. 5. Transposon-chromatin gene fusions. a, Number of candidate fusion genes classified by the level of gene model validation evidence, based on contiguity of the gene model over the genome assembly (i.e. lack of poly-N stretches in the genomic region between the TE- and chromatin-associated domains), evidence of expression, and evidence of contiguous expression (see inset at the right). b, Summary of candidate gene fusions within each chromatin-associated gene family, divided by orthogroup. For each gene, we indicate their similarity to known TE families, presence of TE-associated domains, the evidence of gene model validity, information on their gene structure (whether they are monoexonic or are located in clusters with other fusion genes), and their species distribution. Species abbreviations can be found in Table S1. Source data available in Supplementary Table 6. 
bioRxiv preprint doi: https://doi.org/10.1101/2021.11.30.470311; this version posted December 1, 2021. The copyright holder for this

preprint (which was not certified by peer review) is the author/funder, who has granted bioRxiv a license to display the preprint in

\section{Supplementary Figure S6} perpetuity. It is made available under aCC-BY-NC 4.0 International license.

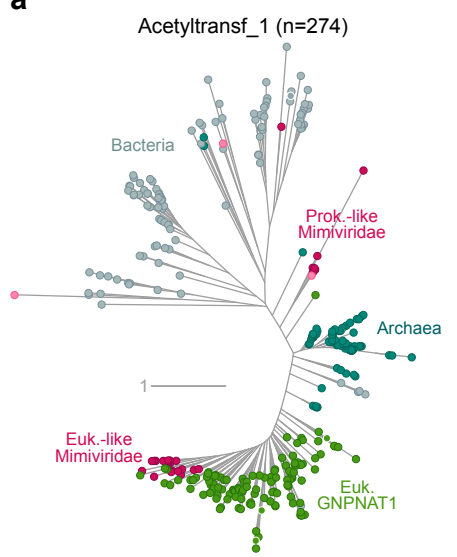

d

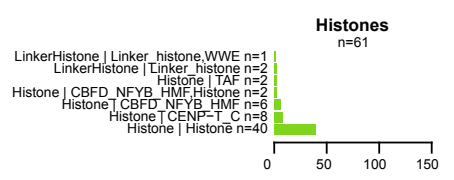

Closest Eukaryota Bacteria

Closest
homologs in:
Archaea b

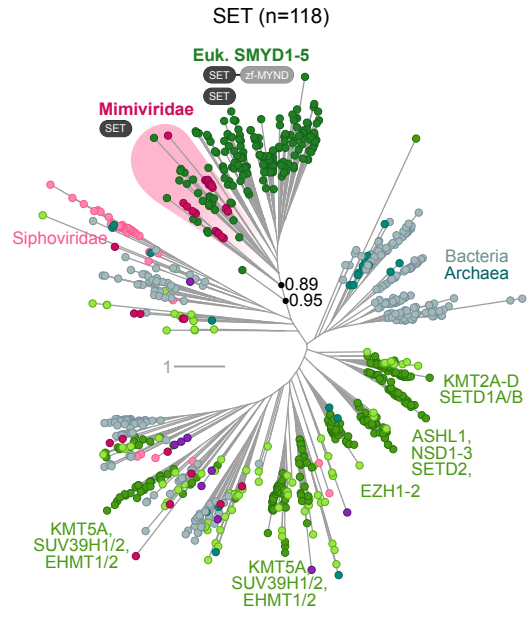

C

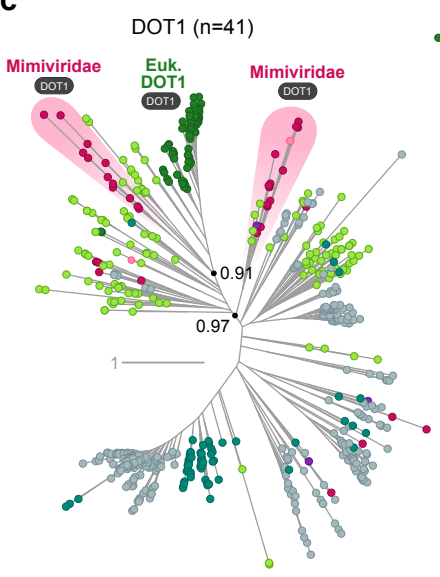
Eukaryote : Mimiviridae
: Archaea
Bacteria

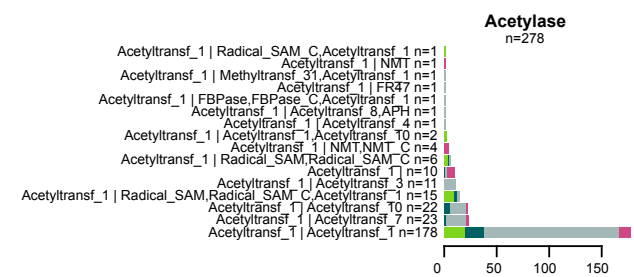

Hist_deacety $\mid$ Hist deacetyln $\begin{gathered}\text { Deacetylase } \\ n=184\end{gathered}$

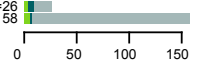

e

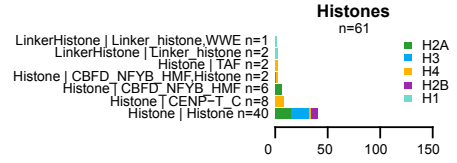

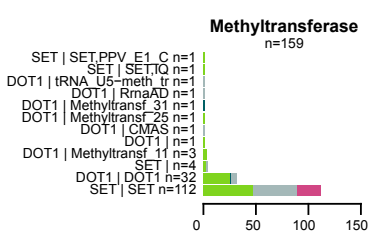
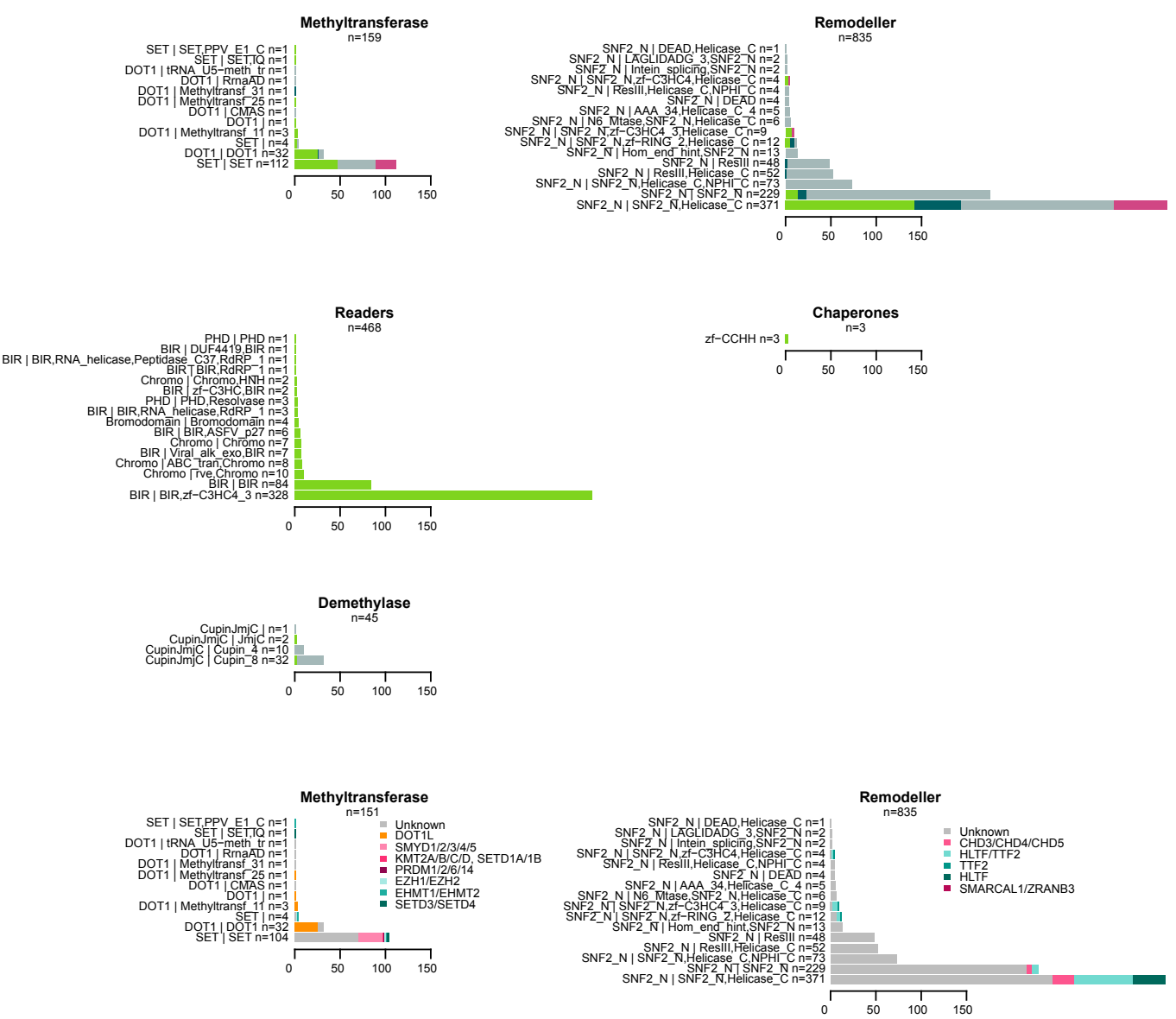
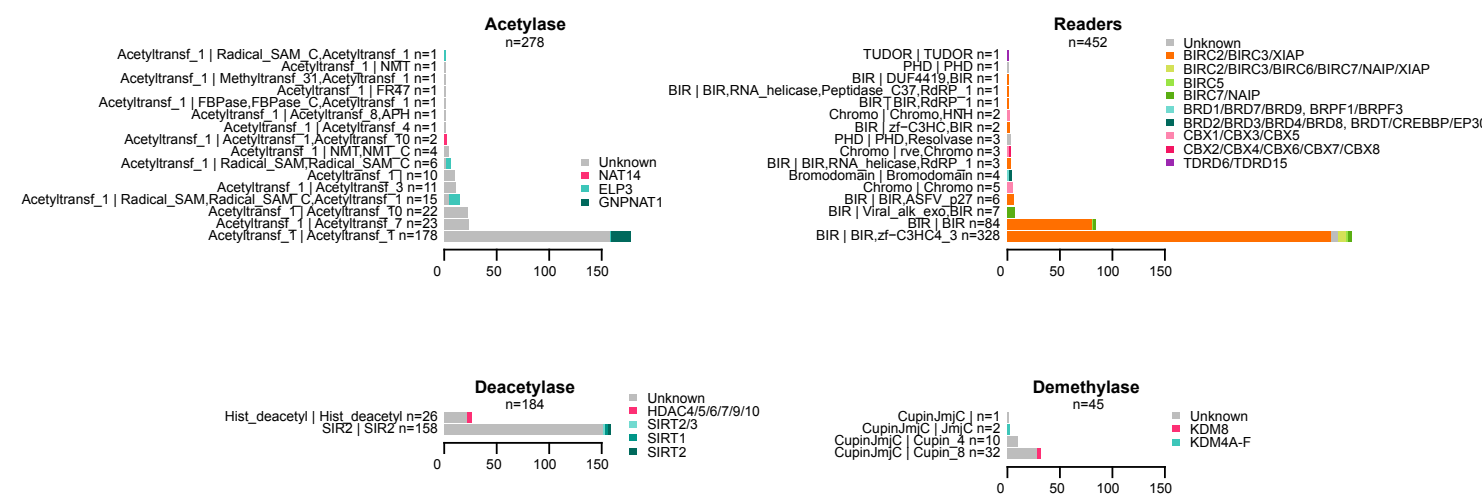
Supplementary Fig. 6. Chromatin proteins in viruses. a-c, Selected gene trees highlighting examples of eukaryotic- and prokaryotic-like viral homologs. $\mathbf{d}$, Number of viral genes of each chromatinassociated gene family, classified according to their closest neighbours from cellular clades in gene tree analyses based on phylogenetic affinity scores (see Methods). Within each gene family, viral sequences are classified according to their PFAM domain architecture - the most common architecture being single-domain in most gene families except for remodellers and BIR readers. e, $I d$., but classifying viral genes according to their phylogenetic affinity to eukaryotic orthology groups. Source data available in Table S6. 


\section{SUPPLEMENTARY MATERIAL AND TABLES LEGENDS}

Supplementary Material 7. Phylogenetic analyses. Collection of gene trees used to identify orthology groups for the eukaryotic chromatin toolkit. UFBS bootstrap supports rare indicated at each node. An annotated eukaryotic species tree is also included.

Supplementary Material 8. Peptide sequences. Collection of peptide sequences used to build gene trees of the eukaryotic chromatin toolkit.

Supplementary Table 1. Taxon sampling. a, List of eukaryotic species used in the comparative genomic analyses, including species abbreviations, data sources for genome or transcriptome assemblies and annotations, and their taxonomic classification. $\mathbf{b}$, List of gene expression datasets (SRA accession numbers) used for gene model validation analyses of candidate fusion genes.

Supplementary Table 2. Histone clusters and classification. a, Pairs of collinear histone-encoding genes, including their genomic coordinates and relative orientation. $\mathbf{b}$, List and sequences of archaeal HMfB histones with N-terminal tails (at least 10 aa before a complete globular domain).

Supplementary Table 3. hPTM conservation. a-g, Table of hPTMs identified in histones of the 26 eukaryotic species used in the comparative proteomics analysis, separated by histone type (canonical and major variants: $\mathrm{H} 2 \mathrm{~A}, \mathrm{H} 2 \mathrm{~B}, \mathrm{H} 3, \mathrm{H} 4$, macroH2A, H2AZ, and H1). Each entry corresponds to a modified peptide, for which we specify modification coordinates along the peptide and relative to the consensus histone sequence (if available). These tables also include entries for hPTMs reported in the literature (indicated as a cited source or as a specific UNIPROT entry; see Methods for a list of sources); in these cases, source peptides and associated data may not be available. h, hPTMs in Archaea.

Supplementary Table 4. Gene family analysis. a, List of gene classes analyzed in the comparative genomics analyses, including the PFAM protein domains used to retrieve homologs and search parameters. b, List of transposon-associated PFAM domains surveyed in the analyses of transposonchromatin gene fusions.

Supplementary Table 5. Evolution of the chromatin machinery in eukaryotes. a, Summary of gene family evolutionary patterns in eukaryotes $(n=1,713$ orthogroups). For each orthogroup, we indicate its gene and functional class, the number of members, species where it is present, and major eukaryotic lineages (Amoebozoa, Opisthokonta+Breviatea+Apusozoa, CRuMs, Ancyromonadida, Mala-wimonadidae, Archaeplastida+Cryptista, SAR+Haptista, Hemimastigophora, Discoba, and Metamonada), the probability of presence at the last eukaryotic common ancestor, the phylogenetic affinity of their closest homologs (other eukaryotic orthogroups, bacteria, archaea or viruses) and their average frequency amongst the 10 nearest neighbours of its member gene in phylogenetic trees ('Phylogenetic affinity score', see Methods); as well as its consensus protein domain architecture (present in at least $25 \%$ of its members). We also indicate the gene symbols of members from four model species: H. sapiens, D. melanogaster, S. cerevisiae, and A. thaliana. b-c, Probability of gain and loss of each gene family at extant and ancestral nodes along the eukaryotic phylogeny. d, Orthogroup assignments per gene. 
Supplementary Table 6. Transposon fusions and viral homology. a, List of candidate fusions between chromatin-associated genes and transposons, including the phylogenetic classification of each gene (orthogroup), protein domain architectures, and the transcriptomics-level and gene model-level evidence supporting each fusion. b, List of chromatin-associated genes encoded by viral genomes, including their species of origin and a summary of their phylogenetic embedding among cellular species (specifically, which are its closest homologs in cellular genomes and the fraction of phylogenetic nearest neighbours they represent, the closest eukaryotic gene family among those close to eukaryotic genes in the gene trees, and the distance to the closest cellular homolog). 


\section{Methods}

\section{Eukaryotic cell culture and tissue sources}

Capsaspora owczarzaki strain ATCC30864 filopodial cells were grown axenically in $5 \mathrm{ml}$ flasks with ATCC medium 1034 (modified PYNFH medium) in an incubator at $23^{\circ} \mathrm{C}$ (Sebé-Pedrós et al., 2013a). Corallochytrium limacisporum strain India was axenically grown in Difco Marine Broth medium at $23^{\circ} \mathrm{C}$, Creolimax fragrantissima strain $\mathrm{CH} 2$ was axenically grown in Difco Marine Broth medium at $12^{\circ} \mathrm{C}$, Spizellomyces punctatus strain DAOM BR 117 was axenically grown in $(0,5 \%$ yeast extract, $3 \%$ glycerol, $\left.1 \mathrm{~g} / \mathrm{L} \mathrm{K} \mathrm{HPO}_{4}, 0,5 \% \mathrm{EtOH}\right)$ medium at $17^{\circ} \mathrm{C}$, Thecamonas trahens strain ATCC50062 was grown in ATCC medium: 1525 Seawater 802 medium, Chlamydomonas reinhardtii strain CC$503 \mathrm{cw} 92 \mathrm{mt}+$ was axenically grown in Gibco TAP medium at $29^{\circ} \mathrm{C}$, Guillardia theta strain CCMP2712 was axenically grown in $\mathrm{L} 1+500 \mathrm{uM} \mathrm{NH} \mathrm{N}_{4} \mathrm{Cl}$ medium at $18^{\circ} \mathrm{C}$, Emiliania huxleyi strain CCMP1516 was grown in L1-Si medium at $18^{\circ} \mathrm{C}$, Thalassiosira pseudonana strain CCMP1335 was axenically grown in $\mathrm{L} 1$ medium at $18^{\circ} \mathrm{C}$, Bigelowiella natans strain CCMP2755 was axenically grown in L1-Si medium at $23^{\circ} \mathrm{C}$, Naegleria gruberi strain ATCC30224 was axenically grown in ATCC medium 1034 (modified PYNFH medium) at $29^{\circ} \mathrm{C}$, Gefionella okellyi strain 249 was grown in $15 \%$ Water Complete Cereal Grass Media (WC-CGM3) at $18^{\circ} \mathrm{C}$ and Fabomonas tropica strain NYK3C was grown in $\mathrm{L} 1+\mathrm{YT}$ medium at $18^{\circ} \mathrm{C}$. All cells were grown in $250 \mathrm{ml}$ culture flasks.

In addition, we used frozen tissues/cells from the following species: Homo sapiens (ES cells, courtesy of Cecilia Ballaré, CRG), Physcomitrella patens (strain Gransden 2004, vegetative stage, courtesy of Josep Casacuberta, CRAG-CSIC), Sycon ciliatum (adult sponges sampled from Bergen, Norway, courtesy of Maja Adamska, ANU) and Phytophthora infestans (strain T30-4, courtesy of Harold J.G.Meijer, Wageningen University).

\section{Archaeal cell culture}

Cultures of Methanobrevibacter cuticularis DSM 11139, Methanospirillum stamsii DSM 26304 and Methanosarcina spelaei DSM 26047 were purchased from the Deutsche Stammsammlung von Mikroorganismen und Zellkulturen GmbH (DSMZ), Braunschweig, Germany. Cultures were grown in closed batch in $50 \mathrm{~mL}$ of defined media in $120 \mathrm{~mL}$ serum bottles (La-Pha-Pack, Langerwehe, Germany). Growth was monitored as OD (600 nm; Analytik Jena, Specord 200 plus). Methanobrevibacter cuticularis was grown in modified Methanobrevibacter cuticularis medium DSMZ 734a (DSMZ 2014) omitting bovine rumen fluid, yeast extract and Na-resazurin at 1.5 bar overpressure $\mathrm{H}_{2} \mathrm{CO}_{2}(20$ vol.- $\% \mathrm{CO} 2$ in $\mathrm{H}_{2}$ ) at $37^{\circ} \mathrm{C}$. As soon as a change in OD was observed, a constant agitation at 90rpm was applied. Methanospirillum stamsii was grown in modified Methanobacterium medium DSMZ 119 (DSMZ 2017) omitting sludge fluid, yeast extract and Na-resazurin at 1 bar overpressure $\mathrm{H}_{2} \mathrm{CO}_{2}$ (20 vol.- $\% \mathrm{CO} 2$ in $\mathrm{H}_{2}$ ) at $29^{\circ} \mathrm{C}$, under constant agitation at 90rpm. Methanosarcina spelaei was grown in modified Methanosarcina barkeri medium DSMZ 120a (DSMZ 2014) omitting yeast extract and Na-resazurin at 1.5 bar overpressure $\mathrm{H}_{2} \mathrm{CO}_{2}\left(20\right.$ vol.- $\% \mathrm{CO} 2$ in $\left.\mathrm{H}_{2}\right)$ at $33^{\circ} \mathrm{C}$, under constant agitation at 90rpm. All gases were obtained from Air Liquide $\mathrm{GmbH}$, Schwechat, Austria. Nitrososphaera viennensis EN76 was grown in continuous culture in a bioreactor as previously described ${ }^{94}$.

Cells were harvested via centrifugation at $21,000 \times \mathrm{xg} 4^{\circ} \mathrm{C} 1 \mathrm{~h}$ (Thermo scientific, Sorvall Lynx 4000 centrifuge), the supernatant discarded and the resulting pellet resuspended in $1 \mathrm{ml}$ of spent medium, 
followed by another round of centrifugation at $21,000 \times \mathrm{xg} 4^{\circ} \mathrm{C}$ for $1 \mathrm{~h}$ (Eppendorf, Centrifuge 5424R). Pellets were stored at $-70^{\circ} \mathrm{C}$. All archaeal histones were extracted as described below.

\section{Histone acid extraction}

Starting material was a pellet of 50-100M cells (washed once with cold PBS) or a flash-frozen tissue homogenate in liquid nitrogen using a ceramic mortar grinder. Cells were washed first in $10 \mathrm{ml}$ of buffer I (10 mM TrisHCl pH 8, $10 \mathrm{mM} \mathrm{MgCl}_{2}, 0.4 \mathrm{M}$ Sucrose). After $5 \mathrm{~min}$ incubation, samples were centrifuged at $8.000 \mathrm{~g}$ for $20 \mathrm{~min}$ at $4^{\circ} \mathrm{C}$ and supernatant was removed. The resulting pellet was resuspended in $1.5 \mathrm{ml}$ of Buffer II (10 mM TrisHCl pH 8, $10 \mathrm{mM} \mathrm{MgCl}_{2}, 0.25 \mathrm{M}$ Sucrose, $1 \%$ Triton X$100,1 \%$ Igepal Ca-630) and incubated $15 \mathrm{~min}$ on ice. In specific cases, cells at this stage were broken using a $2 \mathrm{ml}$ Dounce homogenizer (with Pestle B) or with a $20 \mathrm{G}$ syringe. Then samples were centrifuged at $15.000 \mathrm{~g}$ for $10 \mathrm{~min}$ at $4^{\circ} \mathrm{C}$ and supernatant was removed. The resulting pellet was then slowly resuspended in $300 \mu \mathrm{L}$ of Buffer III $\left(10 \mathrm{mM}\right.$ TrisHCl pH $8,2 \mathrm{mM} \mathrm{MgCl}_{2}, 1.7 \mathrm{M}$ Sucrose, $1 \%$ Triton $\mathrm{X}-100$ ) and then resulting resuspended nuclei were layered on top of another $300 \mu \mathrm{L}$ of Buffer III. Sample was centrifuged at $20.000 \mathrm{~g}$ for $1 \mathrm{~h}$ at $4^{\circ} \mathrm{C}$ and supernatant was removed, resulting in a nuclear pellet ready for acid histone extraction. All buffers were supplemented with spermidine (1:1000), beta-mercaptoethanol (1:1000), protease inhibitors (1x cOmplete cocktail Roche \#11697498001, 1mM PMSF, 1:2000 Pepstatin), phosphatase inhibitors (1x phoSTOP cocktail Roche \#4906845001) and deacetylase inhibitors (10mM Sodium butyrate).

For samples processed using a high-salt $+\mathrm{HCl}$ extraction protocol ${ }^{95,96}$, the pellet was resuspended in $500 \mu \mathrm{L}$ of High Salt Extraction Buffer $\left(20 \mathrm{mM}\right.$ Tris $\mathrm{HCl} \mathrm{pH} 7.4, \mathrm{CaCl}_{2} 1 \mathrm{M}$ and protease, phosphatase and deacetylase inhibitors, same as above). Sample was incubated on ice for $30 \mathrm{~min}$ and then pure $\mathrm{HCl}$ has added to a final $0.3 \mathrm{~N}$ concentration $(12.82 \mu \mathrm{L}$ to the initial $500 \mu \mathrm{L})$. Samples were incubated for at least $2 \mathrm{~h}$ on a rotor at $4^{\circ} \mathrm{C}$ and then centrifuged at $16.000 \mathrm{~g}$ for $10 \mathrm{~min}$ at $4^{\circ} \mathrm{C}$ to remove cellular/nuclear debris. The resulting supernatant containing solubilized histones was transferred to a clean $1.5 \mathrm{ml}$ tube and Trichloroacetic Acid (TCA) was added drop-wise to $25 \%$ final concentration $(171 \mu \mathrm{L}$ TCA to an approximate initial $513 \mu \mathrm{L}$ sample) and left overnight at $4^{\circ} \mathrm{C}$ to precipitate histones. Samples were then centrifuged at $20.000 \mathrm{~g}$ for $30 \mathrm{~min}$ at $4^{\circ} \mathrm{C}$ and the supernatant removed. The pellet was then washed twice with $500 \mu \mathrm{L}$ of cold acetone and then dried for $20 \mathrm{~min}$ at room temperature. Finally, clean histone pellets were resuspended in $30-50 \mu \mathrm{L}$ of ultrapure water. Protein concentration in the sample was measured using BCA and extraction was examined using an SDS-PAGE protein gel with Coomassie staining.

For samples processed using $\mathrm{H}_{2} \mathrm{SO}_{4}{ }^{96}$, the protocol was exactly the same except that $400 \mu \mathrm{L} 0.4 \mathrm{~N}$ $\mathrm{H}_{2} \mathrm{SO}_{4}$ (freshly diluted) was used instead, with a similar incubation time of at least $2 \mathrm{~h}$ at $44^{\circ} \mathrm{C}$.

\section{Histone chemical derivatization}

Histones samples were quantified by the BCA method and $10 \mu \mathrm{g}$ of each sample were derivatized with propionic anhydride, digested with trypsin and derivatized again with phenylisocyanate as previously described ${ }^{48}$. Briefly, samples were dissolved in $9 \mu \mathrm{L}$ of $\mathrm{H} 2 \mathrm{O}$ and $1 \mu \mathrm{L}$ of triethyl ammonium bicarbonate was added to bring the $\mathrm{pH}$ to 8.5 . The propionic anhydride was prepared by adding $1 \mu \mathrm{L}$ of propionic anhydride to $99 \mu \mathrm{L}$ of $\mathrm{H} 2 \mathrm{O}$ and $1 \mu \mathrm{L}$ of propionic anhydride solution was added immediately to the samples with vortexing and incubation for 2 minutes. The reaction was quenched with 
$1 \mu \mathrm{L}$ of $80 \mathrm{mM}$ hydroxylamine and samples were incubated at room temperature for 20 minutes. Tryptic digestion was performed for $3 \mathrm{~h}$ with $0.1 \mu \mathrm{g}$ trypsin (Promega Sequencing Grade; Madison, WI) per sample. A $1 \% \mathrm{v} / \mathrm{v}$ solution of phenyl isocyanate (PIC) in acetonitrile was freshly prepared and $3 \mu \mathrm{l}$ added to each sample ( $17 \mathrm{mM}$ final concentration) and incubated for $60 \mathrm{~min}$ at $37^{\circ} \mathrm{C}$. Samples were acidified by adding $50 \mu \mathrm{L}$ of $5 \%$ formic acid, vacuum dried and desalted with $\mathrm{C} 18$ ultramicrospin columns (The Nest Group, Inc, Southborough, MA).

\section{Liquid Chromatography-Tandem Mass Spectrometry Sample Acquisition}

A 2- $\mu$ g aliquot of the peptide mixture was analyzed using a LTQ-Orbitrap Fusion Lumos mass spectrometer (Thermo Fisher Scientific, San Jose, CA) coupled to an EASY-nLC 1000 (Thermo Fisher Scientific, San Jose, CA ) with both collision induced dissociation (CID) and high energy collision dissociation (HCD) fragmentation.

Peptides were loaded directly onto the analytical column and were separated by reversed-phase chromatography using a $50-\mathrm{cm}$ column with an inner diameter of $75 \mu \mathrm{m}$, packed with $2 \mu \mathrm{m} \mathrm{C} 18$ particles spectrometer (Thermo Scientific, San Jose, CA, USA) with a 90 min chromatographic gradient. The mass spectrometer was operated in positive ionization mode using a data dependent acquisition method. The "Top Speed" acquisition algorithm determined the number of selected precursor ions for fragmentation.

\section{Mass-spectrometry Data Analysis}

Acquired data were analyzed using the Proteome Discoverer software suite (v2.0, Thermo Fisher Scientific), and the Mascot search engine (v2.6, Matrix Science ${ }^{97}$ ) was used for peptide identification using a double-search strategy. First, data were searched against each organism protein database plus the most common contaminants considering Propionylation on $N$-terminal, Propionylation on Lysines and Phenylisocyanate on $N$-terminal as variable modifications. Then a new database was generated with the proteins identified in the first search, and a second search was done considering Propionylation on $N$-terminal, Propionylation on Lysines, Phenylisocyanate on $N$-terminal, Dimethyl lysine, trimethyl lysine, propionyl + methyl lysine, acetyl lysine, crotonyl lysine as variable modifications. Precursor ion mass tolerance of $7 \mathrm{ppm}$ at the MS1 level was used, and up to 5 missed cleavages for trypsin were allowed. False discovery rate (FDR) in peptide identification was set to a maximum of $5 \%$. The identified peptides were filtered by mascot ion score higher than 20 and only PTMs with a localization score ptmRS ${ }^{98}$ higher than 50 were considered. The raw proteomics data have been deposited to the PRIDE ${ }^{99}$ repository with the dataset identifier PXD000000.

\section{Analysis of hPTM conservation}

Identification of canonical and variant histones. We classified histone protein domains from a database of eukaryotic, prokaryotic and viral sequences (see details below) according to their similarity to known canonical (H2A, H2B, H3, H4) and variant histones (e.g., H2AZ, macroH2A, CENP H3 or H3.3), as well as other gene families with histone-like protein folds (e.g., the transcription factors DR1, DRAP1, NFYB/C, POLE3/4, SOS, TAF, or CHRAC). To that end, we used diamond to perform local alignments of each histone domain against $(i)$ a set of curated histone variants obtained from 
HistoneDB $2.0^{47}$, and (ii) annotated each domain according to the best hit in the reference database, which allowed us to classify histone fold-containing proteins as canonical histones (H2A, H2B, H3, $\mathrm{H} 4)$ or their main variants (H2AZ, macroH2A and CENP). This best-hit strategy performs well in distinguishing canonical histones from each other, as well as each canonical histone from its main variants (H3 from CENP, and H2A from H2AZ and macroH2A; Supplementary Fig. 1a).

Then, we built a graph of pairwise similarity between histones, with edges weighted by the alignment bitscore (discarding edges with bitscore $<20$ ). We created visualisations of each connected component in this graph using the spring layout algorithm implemented in the networkx 2.4 Python library (100 iterations, weighted by alignment bitscore $)^{100}$. We selected the four connected components in the graph that matched the four canonical eukaryotic histones $(\mathrm{H} 2 \mathrm{~A}, \mathrm{H} 2 \mathrm{~B}, \mathrm{H} 3, \mathrm{H} 4$; discarding edges with bitscore $<20$ ), retrieved the protein sequences for each of them, aligned them using mafft (EINS-i mode, 1,000 iterations) ${ }^{101}$, and built phylogenetic trees with IQ-TREE 2.1.0 (-fast mode) ${ }^{102}$.

Identification of hPTM homology. We retrieved the protein sequences of the canonical histones identified in each of the 26 species and we used them for the proteomic analysis of hPTMs, and aligned them using mafft (G-INS-i mode, up to 10,000 refinement iterations). For this subset of species, histone class identity was cross-referenced with the HistoneDB search tool. Then, we manually aligned the peptides mapping onto these proteins to identify the position of each hPTM along a consensus alignment. In the case of $\mathrm{H} 3, \mathrm{H} 4$, and macroH2A, the majority of alignment positions were conserved across most eukaryotes in our dataset, and we used a consensus numbering scheme. In the case of $\mathrm{H} 2 \mathrm{~A}, \mathrm{H} 2 \mathrm{AZ}$, and $\mathrm{H} 2 \mathrm{~B}$, non-conserved insertions and deletions at the N-terminal tail precluded the use of a paneukaryotic numbering scheme. Instead, we reported hPTM positions based on the human homolog (if possible), or relative to taxonomically restricted conserved positions. In cases where position-wise homology could not be established, we grouped multiple amino-acids into stretches of unclear homology, which we report separately from conserved positions (question mark symbols in Fig. 1). The complete list of hPTMs and their position-wise coordinates relative to the consensus alignment is available in Supplementary Table 3.

Furthermore, we also reported the presence (in any position) of modifications in less-conserved histone variants, as well as the linker histone $\mathrm{H} 1$.

In addition to the 19 used in our proteomics survey, we also included previously published hPTM data from the following species: the brown alga Ectocarpus siliculosus ${ }^{42}$, the diatom Phaeodactylum tricornutum $^{103}$, the ciliate Tetrahymena thermophila ${ }^{45}$, the ascomycete Neurospora crassa ${ }^{104}$, Saccharomyces cerevisiae and Schizosaccharomyces pombe ${ }^{45}$, and the plant Arabidopsis thaliana ${ }^{105,106}$. We complemented our own proteomics data using previously published hPTM data from Homo sapiens $^{45,107-110}$ and Capsaspora owczarzaki ${ }^{41}$.

\section{Comparative genomics analysis of chromatin-associated proteins}

Data retrieval. We identified homologs of gene families associated with eukaryotic chromatin, using a database of predicted proteomes from a selection of eukaryotic species from all major supergroups ( $n=172$ species; see Supplementary Table 1 for their taxonomic classification and data sources), as well as archaeal and viral peptides available in the NCBI $n r$ peptide collection (as of 25th of April, 2020) and bacterial peptides available in RefSeq (release 99, 11th May, 2020). The database of viral 
sequences was complemented with peptides from 501 genomes of nucleocytoplasmic large DNA viruses $^{111}$.

Gene family searches. We defined 61 gene classes associated with eukaryotic chromatin, based on HMM models obtained from the Pfam database (release 33.0) ${ }^{112}$. This list included canonical and linker histones ( $n=2$ families), chromatin-specific lysine acetylases $(n=5)$, deacetylases $(n=2)$, methyltransferases $(n=2)$, demethylases $(n=2)$, chromatin readers $(n=16)$, remodellers $(n=1)$ and chaperones $(n=13)$, as well as multiple families associated with the Polycomb complexes $(n=18)$. The complete list of gene families, including the associated HMM models, is available in Supplementary Table 4.

For each gene family, we retrieved all homologs from the eukaryotic, archaeal, bacterial and viral databases using the hmmsearch tool from the HMMER 3.3 toolkit ${ }^{113}$ and the gathering threshold defined in each Pfam HMM model. We recorded the taxonomic profile of each homolog.

Orthology identification. We aimed to identify groups of orthologs within each of the 61 chromatinassociated gene families using targeted phylogenetic analyses. We followed the following strategy for each of the 59 sets of eukaryotic genes. First, we partitioned each set into one or more homology groups based on pairwise local sequence alignments using diamond 0.9.36.137 (high sensitivity allto-all search) ${ }^{114}$, followed by clustering of the resulting pairwise alignments graph with MCL 14.137 (--abc mode $)^{115}$, using low inflation values (see Supplementary Table 4) to favour inclusive groupings. Second, we performed multiple sequence alignments of each homology group with mafft $7.471^{101}$ under the E-INS-i mode (optimised for multiple conserved regions), running up to 10,000 refinement iterations. Third, we trimmed the resulting multiple sequence alignments using clip-kit 0.1 (kpic-gappy mode $)^{116}$. Fourth, we built phylogenetic trees for each trimmed alignment using IQ-TREE 2.1.0 $0^{102}$, selecting the best-fitting evolutionary model using its ModelTest module (according to the Bayesian Information Criterion) and using 1,000 UFBS bootstrap supports ${ }^{117}$. Each tree was run for up to 10,000 iterations until convergence was attained (at the 0.999 correlation coefficient threshold, and for at least 200 iterations).

Then, we parsed the species composition of each gene tree in order to identify groups of orthologous proteins using the POSSVM pipeline ${ }^{118}$. Specifically, we used the species overlap algorithm ${ }^{119}$ implemented in the ETE toolkit 3.1.1 ${ }^{120}$, which identifies pairs of orthologous genes in a phylogenetic tree by examining the species composition of each subtree, and classifying internal nodes as paralogy nodes (if there is overlap in the species composition between each of its two descendant subtrees) or orthology nodes (if there is no overlap). Pairs of genes linked by an orthology node are then recorded as orthology pairs. In our analysis, we used an overlap threshold=0 (i.e. any species composition overlap between the two descendant subtrees is classified as a paralogy event). The resulting list of pairwise orthology relationships between genes was clustered into groups of orthologs (orthogroups) using MCL. We further annotated each orthogroup with a string denoting the gene symbols of the human proteins therein (if any).

Overall, we classified 51,426 proteins from 61 gene classes (defined by protein structural domains), divided into 242 gene trees and 1,713 gene families (orthogroups). The source peptide sequences and gene trees used for these analyses are available in Supplementary Material 7 and 8. 
Ancestral reconstruction of gene content. We inferred the presence, gain and loss of each orthogroup along the eukaryotic tree of life, using a phylogenetic birth-and-death model ${ }^{121}$ implemented in Count ${ }^{122}$. This tool takes a numeric profile of gene family presence/absence in extant species (172 in our dataset) and a phylogenetic tree defining their evolutionary relationships, and infers the probabilities of gain and loss of each family at each ancestral node along the tree.

First we trained the probabilistic model in Count. As a training set, we used a random sample of 1,000 PFAM domains annotated in the 172 species of interest (restricting the sampling to domains present in at least $5 \%$ of species). The final model consists of gain, loss and transfer rates with two $\Gamma$ categories each, and a constant duplication rate (given that we only recorded gene presence/absence, duplication events are not included in our downstream analyses). This model was obtained in three sequential rounds of training, so as to sequentially add zero, one and two $\Gamma$ categories to each evolutionary rate. Each round consisted of up to 100 iterations, and stopped when the relative change in the model log-likelihood fell by $0.1 \%$ in two consecutive rounds. The final evolutionary rates and the Newick-formatted species tree used in this step are available in the Supplementary Table 1 and Supplementary Fig. 3a.

Second, we calculated the posterior probability of gain, loss and presence of each orthogroup in our dataset with Count. The aggregated counts of gains and losses of the various classes of chromatinassociated proteins (acetylases, deacetylases, methyltransferases, demethylases, readers and remodellers) along the eukaryotic tree were obtained by summing the probabilities of gain, presence or loss of all orthogroups of a given class at each ancestral node. To investigate the evolutionary histories of specific orthogroups at a given node in the tree, we applied a probability threshold of 0.9 (for presence) or 0.5 (to identify the most probable gain and loss node). The Count model was not able to calculate ancestral probabilities for a few orthogroups with widespread phylogenetic distributions, due to violations of the birth-and-death model ( 25 out of 1,713 families). In order to be able to report presence probabilities in the LECA for these orthogroups, we inferred their presence in this ancestor using the Wagner parsimony procedure implemented in Count with a gain-to-loss penalty $g=5$, and recorded their presence as binary values (0/1) accordingly.

Protein domain architecture analyses. We annotated the Pfam domains present in each protein from the gene classes listed in Supplementary Table 4, using Pfamscan 1.6-3 and the Pfam 33.0 database $^{112}$. We visualized the networks of protein domain co-occurrence from the point of view of the core domain(s) that define each gene class, using the networkx Python library (version 2.4) ${ }^{100}$. Specifically, we built a graph where each node represented 'accessory' domains (i.e. domains that cooccur with the 'core' domain that defines given gene class), node size reflected number of co-occurrences with the 'core' domain, and edges reflected co-occurrences between accessory domains. We identified communities of frequently co-occurring accessory domains using the label propagation algorithm implemented in networkx (communities submodule), which we used as a basis to manually annotate groups of co-occurring domains of interest (Fig. 5C). Network visualizations were created using the NEATO spring layout algorithm from the Graphviz 2.40.1 Python library ${ }^{123}$.

In parallel, we also recorded the presence of Pfam domains within individual orthogroups, and their taxonomic distribution. 
Prokaryotic roots of the eukaryotic chromatin machinery. We retrieved all eukaryotic domains from gene class shared with prokaryotes (Histones, Acetyltransf_1, GNAT_acetyltr_2, MOZ_SAS, Hist_deacetyl, SIR2, DOT1, SET, CupinJmjC, ING, MBT, PWWP and SNF2_N), collapsing identical sequences at $100 \%$ similarity with $C D$-HIT 4.8.1 ${ }^{124}$, and identified their closest homologs amongst the corresponding archaea and bacteria protein domain sets, using diamond local alignments (high sensitivity search). The archaeal and bacterial protein sets were also reduced with CD-HIT (at 95\% and $90 \%$ sequence similarity, respectively). Each set of sequences was then partitioned into lowgranularity homology clusters using the $M C L$-based strategy described above (inflation $I=1.2$ ), and a phylogenetic tree was then constructed from each homology cluster with IQ-TREE (as described above).

Then, we mapped each eukaryotic gene to its orthogroup (obtained from eukaryotic-only analyses, see above) and used the distribution of phylogenetic distances from the prokaryotic+eukaryotic gene trees to classify them according to their similarity to ( $i$ ) eukaryotic genes in other orthogroups, (ii) archaeal homologs, or (iii) bacterial homologs. Specifically, we used a majority-voting procedure in which we recorded the number of sequences of eukaryotic, archaeal or bacterial origin amongst the ten nearest neighbors of each gene (measuring intergenic distances as substitutions per site), and assigned the most common taxonomic group as the 'closest' homolog of that gene (minimum 50\% agreement). This fraction is termed 'Phylogenetic affinity score' and reported in Supplementary Table 5. The pairwise distances were obtained from each gene tree using the cophenetic distance method in the cophenetic.phylo utility of the ape $5.4 R$ library ${ }^{125}$.

Characterisation of fusions with transposon-associated domains. We retrieved all classified genes from our eukaryotic dataset that contained transposon-associated Pfam domains (version 33.0), using a list compiled from ${ }^{62,126}$ (complete list in Supplementary Table 4), totaling 823 candidate fusions from 91 species (listed in Supplementary Table 6). We annotated these genes to their most similar known TE element by aligning them against the Dfam 3.3 database ${ }^{127}$ using the tblastn program in BLAST 2.2.31 $1^{128}$.

We validated each candidate fusion using the following criteria: $(i)$ contiguity of the gene model on the genome assembly, i.e., recording which genes were interrupted by poly- $N$ stretches (which might indicate an incorrect gene model); (ii) evidence of expression in at least one sample from a range of publicly available transcriptomic experiments (from the NCBI SRA repository); (iii) evidence of contiguous expression, i.e., whether an expressed transcript had mapped reads along the entire region located between the 'core' and 'TE-associated' domains; (iv) we also recorded the number of exons per gene; and $(v)$ located near any other candidate fusion gene in the genome.

The list of SRA experiments used for these validation steps is available in Supplementary Table 1. This list includes 64 out of 91 species for which transcriptomics datasets are publicly available, and covers 768 out of the 822 TE fusion candidates (93\%). RNA-seq read mapping was performed with the quant tool in salmon 1.3.0 ${ }^{129}$ using the complete set of spliced transcripts of each species as the reference database (built with $k$-mer length $=31$ ). We used bedtools $2.29 .2^{130}$ to identify poly- $N$ stretches in the genome assembly (assembly contiguity criterion). We identified regions of zero coverage along the transcript sequence (expression contiguity criterion) using the bedtools genomecov 
utility, and clusters of close candidate fusions genes using bedtools cluster (at a fixed distance $=100$ $\mathrm{kbp})$.

Analysis of viral homologs. We investigated the homology of the viral chromatin-associated genes (which included 19 out of 61 families present in our survey) using joint phylogenetic analyses of protein domains from virus, prokaryotic and eukaryotic genes. We used the same method described above to investigate the prokaryotic roots of eukaryotic gene classes: we aligned viral domains against a database of cellular homologs (high sensitivity diamond search), followed by low-granularity $M C L$ clustering (inflation $I=1.2$ ) and phylogenetic tree building (IQ-TREE). Then, we used the same majority-voting procedure described above to classify viral homologs according to their similarity to eukaryotic, archaeal or bacterial gene families based on their distribution of phylogenetic distances. For viral genes that were most similar to eukaryotic genes, we used the same procedure to map them to their closest eukaryotic orthogroup.

The complete list of viral genes and their phylogenetic annotation is available in Supplementary Table 6. Out of 2,163 viral genes in our dataset, 2,144 could be annotated as similar to a particular cellular group using this procedure (99.1\%), and the majority of these genes had a high agreement in the annotations of their nearest neighbors (2,096 with $\geq 50 \%$ agreement; 1,449 with $\geq 90 \%$ agreement).

In the case of viral histones, we built a separate phylogeny with a few modifications in our protocol: (i) we used additional viral genes obtained from ${ }^{65}$ as a reference; (ii) we omitted the CD-HIT reduction and $M C L$ partitioning steps, and jointly analyzed the entire set of homologs instead; and (iii) in the phylogenetic reconstruction step, we used the approximate Bayes posterior probabilities ${ }^{131}$ implemented in IQ-TREE.

Identification of archaeal $N$-terminal histone tails. We retrieved all archaeal histone domains classified belonging to the HMfB-like connected component in Fig. 1b, and retained those that fulfilled the following criteria: (i) contained a complete CBFD_NFYB_HMF domain according to the hmmscan search (defined as an alignment starting at least at the 10th position of the HMM model, and up to the 55 th position; the HMM model contains 65 positions); and (ii) the predicted tail ( $N$-terminal to the core domain boundaries defined by hmmscan) was at least 10 residues long. 84 genes passed these filters, including three $N$-terminal containing histones previously identified by Henneman et al..$^{54}$. A complete list is available in Supplementary Table 2. We manually examined the sequences of archaeal tails and aligned four sets of similar histones with mafft G-INS-i (Supplementary Fig. 1d). Alignments were plotted using the $m s a$ 1.24.0 library in $R^{132}$.

\section{Data and Code Availability}

The mass spectrometry proteomics data have been deposited to the ProteomeXchange Consortium via the PRIDE partner repository with the dataset identifier PDX00000 (accession code pending). Code for reproducing the analysis is available in our lab Github repository (https://github.com/sebepedroslab/chromatin-evolution-analysis). 


\section{References}

1. Struhl, K. Fundamentally different logic of gene regulation in eukaryotes and prokaryotes. Cell 98, 1-4 (1999).

2. Kornberg, R. D. \& Lorch, Y. Primary Role of the Nucleosome. Mol. Cell 79, 371-375 (2020).

3. Jenuwein, T. \& Allis, C. D. Translating the Histone Code. Science (80-. ). 293, 1074-1080 (2001).

4. Berger, S. L. The complex language of chromatin regulation during transcription. Nature 447, 407-12 (2007).

5. Banaszynski, L. a, Allis, C. D. \& Lewis, P. W. Histone variants in metazoan development. Dev. Cell 19, 662-74 (2010).

6. Allis, C. D. \& Jenuwein, T. The molecular hallmarks of epigenetic control. Nat. Rev. Genet. 1, (2016).

7. Sultana, T. et al. The Landscape of L1 Retrotransposons in the Human Genome Is Shaped by Preinsertion Sequence Biases and Post-insertion Selection. Mol. Cell 74, 555-570.e7 (2019).

8. Gangadharan, S., Mularoni, L., Fain-Thornton, J., Wheelan, S. J. \& Craig, N. L. DNA transposon Hermes inserts into DNA in nucleosome-free regions in vivo. Proc. Natl. Acad. Sci. 107, 21966-21972 (2010).

9. Shinn, P. et al. HIV-1 Integration in the Human Genome Favors Active Genes and Local Hotspots. Cell 110, 521-529 (2002).

10. Goodier, J. L. Restricting retrotransposons: a review. Mob. DNA 7, 16 (2016).

11. Molaro, A. \& Malik, H. S. Hide and seek: how chromatin-based pathways silence retroelements in the mammalian germline. Curr. Opin. Genet. Dev. 37, 51-58 (2016).

12. Malik, H. S. \& Henikoff, S. Phylogenomics of the nucleosome. Nat. Struct. Biol. 10, 882-91 (2003).

13. Talbert, P. B. \& Henikoff, S. Histone variants--ancient wrap artists of the epigenome. Nat. Rev. Mol. Cell Biol. 11, 264-75 (2010).

14. Soboleva, T. a., Nekrasov, M., Ryan, D. P. \& Tremethick, D. J. Histone variants at the transcription start-site. Trends Genet. 30, 199-209 (2014).

15. Zink, L.-M. \& Hake, S. B. Histone variants: nuclear function and disease. Curr. Opin. Genet. Dev. 37, 82-89 (2016).

16. Weber, C. M. \& Henikoff, S. Histone variants: dynamic punctuation in transcription. Genes Dev. 28, 672-82 (2014).

17. Zentner, G. E. \& Henikoff, S. Regulation of nucleosome dynamics by histone modifications. Nat. Struct. Mol. Biol. 20, 259-66 (2013).

18. Campos, E. I. \& Reinberg, D. Histones: annotating chromatin. Annu. Rev. Genet. 43, 559-99 (2009).

19. Strahl, B. D. \& Allis, C. D. The language of covalent histone modifications. Nature 403, 41-45 (2000).

20. Bannister, A. J. \& Kouzarides, T. Regulation of chromatin by histone modifications. Cell Res. 21, 38195 (2011).

21. Talbert, P. B. \& Henikoff, S. The Yin and Yang of Histone Marks in Transcription. Annu. Rev. Genomics Hum. Genet. 22, 147-170 (2021).

22. Taverna, S. D., Li, H., Ruthenburg, A. J., Allis, C. D. \& Patel, D. J. How chromatin-binding modules interpret histone modifications: lessons from professional pocket pickers. Nat. Struct. Mol. Biol. 14, $1025-40$ (2007).

23. Musselman, C. a, Lalonde, M.-E., Côté, J. \& Kutateladze, T. G. Perceiving the epigenetic landscape through histone readers. Nat. Struct. Mol. Biol. 19, 1218-27 (2012). 
24. Gurard-Levin, Z. a, Quivy, J.-P. \& Almouzni, G. Histone Chaperones: Assisting Histone Traffic and Nucleosome Dynamics. Annu. Rev. Biochem. 83, 487-517 (2014).

25. Burgess, R. J. \& Zhang, Z. Histone chaperones in nucleosome assembly and human disease. Nat. Struct. Mol. Biol. 20, 14-22 (2013).

26. Koster, M. J. E., Snel, B. \& Timmers, H. T. M. Genesis of Chromatin and Transcription Dynamics in the Origin of Species. Cell 161, 724-736 (2015).

27. Hargreaves, D. C. \& Crabtree, G. R. ATP-dependent chromatin remodeling: genetics, genomics and mechanisms. Cell Res. 21, 396-420 (2011).

28. Gornik, S. G. et al. Loss of nucleosomal DNA condensation coincides with appearance of a novel nuclear protein in dinoflagellates. Curr. Biol. 22, 2303-12 (2012).

29. Mattiroli, F. et al. Structure of histone-based chromatin in Archaea. Science (80-. ). 357, 609-612 (2017).

30. Warnecke, T., Becker, E. a, Facciotti, M. T., Nislow, C. \& Lehner, B. Conserved substitution patterns around nucleosome footprints in eukaryotes and Archaea derive from frequent nucleosome repositioning through evolution. PLoS Comput. Biol. 9, e1003373 (2013).

31. Ammar, R. et al. Chromatin is an ancient innovation conserved between Archaea and Eukarya. Elife 1, e00078 (2012).

32. Rojec, M., Hocher, A., Merkenschlager, M. \& Warnecke, T. Chromatinization of Escherichia coli with archaeal histones. bioRxiv 660035 (2019) doi:10.1101/660035.

33. Forbes, A. J. et al. Targeted analysis and discovery of posttranslational modifications in proteins from methanogenic archaea by top-down MS. Proc. Natl. Acad. Sci. U. S. A. 101, 2678-83 (2004).

34. Weidenbach, K. et al. Deletion of the archaeal histone in Methanosarcina mazei Gö1 results in reduced growth and genomic transcription. Mol. Microbiol. 67, 662-671 (2008).

35. Talbert, P. B., Meers, M. P. \& Henikoff, S. Old cogs, new tricks: the evolution of gene expression in a chromatin context. Nat. Rev. Genet. (2019) doi:10.1038/s41576-019-0105-7.

36. de Mendoza, A. \& Sebe-Pedros, A. Origin and evolution of eukaryotic transcription factors. Curr. Opin. Genet. Dev. 59, 25-32 (2019).

37. Schwaiger, M. et al. Evolutionary conservation of the eumetazoan gene regulatory landscape. Genome Res. 24, 639-650 (2014).

38. Sebé-Pedrós, A. et al. Early metazoan cell type diversity and the evolution of multicellular gene regulation. Nat. Ecol. Evol. 2, 1176-1188 (2018).

39. Connolly, L. R., Smith, K. M. \& Freitag, M. The Fusarium graminearum Histone H3 K27 Methyltransferase KMT6 Regulates Development and Expression of Secondary Metabolite Gene Clusters. PLoS Genet. 9, e1003916 (2013).

40. Jamieson, K., Rountree, M. R., Lewis, Z. a, Stajich, J. E. \& Selker, E. U. Regional control of histone H3 lysine 27 methylation in Neurospora. Proc. Natl. Acad. Sci. 110, 6027-6032 (2013).

41. Sebé-Pedrós, A. et al. The Dynamic Regulatory Genome of Capsaspora and the Origin of Animal Multicellularity. Cell 165, 1224-1237 (2016).

42. Bourdareau, S. et al. Histone modifications during the life cycle of the brown alga Ectocarpus. Genome Biol. 22, 12 (2021).

43. Wang, S. Y. et al. Role of epigenetics in unicellular to multicellular transition in Dictyostelium. Genome Biol. 22, 134 (2021).

44. Taverna, S. D., Coyne, R. S. \& Allis, C. D. Methylation of Histone H3 at Lysine 9 Targets Programmed DNA Elimination in Tetrahymena University of Virginia Health System. Cell 110, 701-711 (2002). 
45. Garcia, B. a et al. Organismal differences in post-translational modifications in histones $\mathrm{H} 3$ and H4. J. Biol. Chem. 282, 7641-55 (2007).

46. Drinnenberg, I. A. et al. EvoChromo: towards a synthesis of chromatin biology and evolution. Development 146, dev178962 (2019).

47. Draizen, E. J. et al. HistoneDB 2.0: a histone database with variants-an integrated resource to explore histones and their variants. Database 2016, baw014 (2016).

48. Maile, T. M. et al. Mass Spectrometric Quantification of Histone Post-translational Modifications by a Hybrid Chemical Labeling Method. Mol. Cell. Proteomics 14, 1148-1158 (2015).

49. Li, B., Carey, M. \& Workman, J. L. The Role of Chromatin during Transcription. Cell 128, 707-719 (2007).

50. Rajagopal, N. et al. Distinct and Predictive Histone Lysine Acetylation Patterns at Promoters, Enhancers, and Gene Bodies. G3 Genes|Genomes|Genetics 4, 2051-2063 (2014).

51. Koonin, E. V. \& Yutin, N. The Dispersed Archaeal Eukaryome and the Complex Archaeal Ancestor of Eukaryotes. Cold Spring Harb. Perspect. Biol. 6, a016188-a016188 (2014).

52. Sandman, K. \& Reeve, J. N. Archaeal histones and the origin of the histone fold. Curr. Opin. Microbiol. 9, 520-5 (2006).

53. Pereira, S. L., Grayling, R. a, Lurz, R. \& Reeve, J. N. Archaeal nucleosomes. Proc. Natl. Acad. Sci. U. S. A. 94, 12633-7 (1997).

54. Henneman, B., van Emmerik, C., van Ingen, H. \& Dame, R. T. Structure and function of archaeal histones. PLOS Genet. 14, e1007582 (2018).

55. Imachi, H. et al. Isolation of an archaeon at the prokaryote-eukaryote interface. bioRxiv 726976 (2019) doi:10.1101/726976.

56. Alva, V. \& Lupas, A. N. Histones predate the split between bacteria and archaea. Bioinformatics 35, 2349-2353 (2019).

57. Allis, C. D. et al. New Nomenclature for Chromatin-Modifying Enzymes. Cell 131, 633-636 (2007).

58. Dion, M. F., Altschuler, S. J., Wu, L. F. \& Rando, O. J. Genomic characterization reveals a simple histone H4 acetylation code. Proc. Natl. Acad. Sci. 102, 5501-5506 (2005).

59. de Jong, J. et al. Chromatin Landscapes of Retroviral and Transposon Integration Profiles. PLoS Genet. 10, e1004250 (2014).

60. Sultana, T., Zamborlini, A., Cristofari, G. \& Lesage, P. Integration site selection by retroviruses and transposable elements in eukaryotes. Nat. Rev. Genet. 18, 292-308 (2017).

61. Gao, X., Hou, Y., Ebina, H., Levin, H. L. \& Voytas, D. F. Chromodomains direct integration of retrotransposons to heterochromatin. Genome Res. 18, 359-369 (2008).

62. Cosby, R. L. et al. Recurrent evolution of vertebrate transcription factors by transposase capture. Science (80-. ). 371, eabc6405 (2021).

63. Cordaux, R., Udit, S., Batzer, M. A. \& Feschotte, C. Birth of a chimeric primate gene by capture of the transposase gene from a mobile element. Proc. Natl. Acad. Sci. 103, 8101 LP - 8106 (2006).

64. Fiedler, M. et al. Decoding of Methylated Histone H3 Tail by the Pygo-BCL9 Wnt Signaling Complex. Mol. Cell 30, 507-518 (2008).

65. Erives, A. J. Phylogenetic analysis of the core histone doublet and DNA topo II genes of Marseilleviridae: evidence of proto-eukaryotic provenance. Epigenetics Chromatin 10, 55 (2017).

66. Liu, Y. et al. Virus-encoded histone doublets are essential and form nucleosome-like structures. Cell 184, 4237-4250.e19 (2021). 
67. Valencia-Sánchez, M. I. et al. The structure of a virus-encoded nucleosome. Nat. Struct. Mol. Biol. 28, 413-417 (2021).

68. Iyer, L. M., Balaji, S., Koonin, E. V \& Aravind, L. Evolutionary genomics of nucleo-cytoplasmic large DNA viruses. Virus Res. 117, 156-184 (2006).

69. Nagamine, T. Apoptotic arms races in insect-baculovirus coevolution. Physiol. Entomol. phen.12371 (2021) doi:10.1111/phen.12371.

70. Starrett, G. J. et al. Adintoviruses: An Animal-Tropic Family of Midsize Eukaryotic Linear dsDNA (MELD) Viruses. bioRxiv 697771 (2020) doi:10.1101/697771.

71. Hocher, A. et al. Growth temperature is the principal driver of chromatinization in archaea. bioRxiv 2021.07.08.451601 (2021) doi:10.1101/2021.07.08.451601.

72. Eme, L., Spang, A., Lombard, J., Stairs, C. W. \& Ettema, T. J. G. Archaea and the origin of eukaryotes. Nat. Rev. Microbiol. 15, 711-723 (2017).

73. Ak1l, C. \& Robinson, R. C. Genomes of Asgard archaea encode profilins that regulate actin. Nature 562, 439-443 (2018).

74. Spang, A. et al. Complex archaea that bridge the gap between prokaryotes and eukaryotes. Nature 521, 173-179 (2015).

75. Koonin, E. V. The origin and early evolution of eukaryotes in the light of phylogenomics. Genome Biol. 11, 209 (2010).

76. Sebé-Pedrós, A., Grau-Bové, X., Richards, T. a \& Ruiz-Trillo, I. Evolution and Classification of Myosins, a Paneukaryotic Whole-Genome Approach. Genome Biol. Evol. 6, 290-305 (2014).

77. Richards, T. A. \& Cavalier-Smith, T. Myosin domain evolution and the primary divergence of eukaryotes. Nature 436, 1113-8 (2005).

78. Wickstead, B., Gull, K. \& Richards, T. Patterns of kinesin evolution reveal a complex ancestral eukaryote with a multifunctional cytoskeleton. BMC Evol. Biol. 10, 110 (2010).

79. Dacks, J. B. \& Field, M. C. Evolution of the eukaryotic membrane-trafficking system: origin, tempo and mode. J. Cell Sci. 120, 2977-85 (2007).

80. Collins, L. \& Penny, D. Complex Spliceosomal Organization Ancestral to Extant Eukaryotes. Mol. Biol. Evol. 22, 1053-1066 (2005).

81. Grau-Bové, X., Sebé-Pedrós, A. \& Ruiz-Trillo, I. The Eukaryotic Ancestor Had a Complex Ubiquitin Signaling System of Archaeal Origin. Mol. Biol. Evol. 32, 726-739 (2015).

82. Kundaje, A. et al. Integrative analysis of 111 reference human epigenomes. Nature 518, 317-330 (2015).

83. Ho, J. W. K. et al. Comparative analysis of metazoan chromatin organization. Nature 512, 449-452 (2014).

84. Montgomery, S. A. et al. Chromatin Organization in Early Land Plants Reveals an Ancestral Association between H3K27me3, Transposons, and Constitutive Heterochromatin. Curr. Biol. 30, 573588.e7 (2020).

85. Frapporti, A. et al. The Polycomb protein Ezl1 mediates H3K9 and H3K27 methylation to repress transposable elements in Paramecium. Nat. Commun. 10, 2710 (2019).

86. Lennartsson, A. \& Ekwall, K. Histone modification patterns and epigenetic codes. Biochim. Biophys. Acta 1790, 863-8 (2009).

87. Peterson, C. L. \& Laniel, M.-A. Histones and histone modifications. Curr. Biol. 14, R546-51 (2004).

88. Rando, O. J. Combinatorial complexity in chromatin structure and function: revisiting the histone code. Curr. Opin. Genet. Dev. 22, 148-155 (2012). 
89. de Mendoza, A., Pflueger, J. \& Lister, R. Capture of a functionally active methyl-CpG binding domain by an arthropod retrotransposon family. Genome Res. 29, 1277-1286 (2019).

90. De Mendoza, A. et al. Recurrent acquisition of cytosine methyltransferases into eukaryotic retrotransposons. Nat. Commun. 9, 1-11 (2018).

91. Ji, X. et al. Chromatin proteomic profiling reveals novel proteins associated with histone-marked genomic regions. Proc. Natl. Acad. Sci. U. S. A. 112, 3841-3846 (2015).

92. Wierer, M. \& Mann, M. Proteomics to study DNA-bound and chromatin-associated gene regulatory complexes. Hum. Mol. Genet. 25, R106-R114 (2016).

93. Villaseñor, R. et al. ChromID identifies the protein interactome at chromatin marks. Nat. Biotechnol. 38, 728-736 (2020).

94. Stieglmeier, M. et al. Nitrososphaera viennensis gen. nov., sp. nov., an aerobic and mesophilic, ammonia-oxidizing archaeon from soil and a member of the archaeal phylum Thaumarchaeota. Int. $J$. Syst. Evol. Microbiol. 64, 2738-2752 (2014).

95. Tirichine, L. et al. Histone extraction protocol from the two model diatoms Phaeodactylum tricornutum and Thalassiosira pseudonana. Mar. Genomics 13, 21-25 (2014).

96. Shechter, D., Dormann, H. L., Allis, C. D. \& Hake, S. B. Extraction, purification and analysis of histones. Nat. Protoc. 2, 1445-57 (2007).

97. Perkins, D. N., Pappin, D. J. C., Creasy, D. M. \& Cottrell, J. S. Probability-based protein identification by searching sequence databases using mass spectrometry data. Electrophoresis 20, 3551-3567 (1999).

98. Taus, T. et al. Universal and confident phosphorylation site localization using phosphoRS. J. Proteome Res. 10, 5354-5362 (2011).

99. Vizcaíno, J. A. et al. 2016 update of the PRIDE database and its related tools. Nucleic Acids Res. 44, D447-56 (2016).

100. Hagberg, A. A., Schult, D. A. \& Swart, P. J. Exploring Network Structure, Dynamics, and Function using NetworkX. in Proceedings of the 7th Python in Science Conference (eds. Varoquaux, G., Vaught, T. \& Millman, J.) 11-15 (2008).

101. Katoh, K. \& Standley, D. M. MAFFT Multiple Sequence Alignment Software Version 7: Improvements in Performance and Usability. Mol. Biol. Evol. 30, 772-780 (2013).

102. Nguyen, L.-T., Schmidt, H. A., von Haeseler, A. \& Minh, B. Q. IQ-TREE: A Fast and Effective Stochastic Algorithm for Estimating Maximum-Likelihood Phylogenies. Mol. Biol. Evol. 32, 268-274 (2015).

103. Veluchamy, A. et al. An integrative analysis of post-translational histone modifications in the marine diatom Phaeodactylum tricornutum. Genome Biol. 16, 102 (2015).

104. Xiong, L., Adhvaryu, K. K., Selker, E. U. \& Wang, Y. Mapping of lysine methylation and acetylation in core histones of neurospora crassa. Biochemistry 49, 5236-5243 (2010).

105. Zhang, K., Sridhar, V. V., Zhu, J., Kapoor, A. \& Zhu, J. K. Distinctive core histone post-translational modification patterns in Arabidopsis thaliana. PLoS One 2, (2007).

106. Johnson, L. et al. Mass spectrometry analysis of Arabidopsis histone H3 reveals distinct combinations of post-translational modifications. Nucleic Acids Res. 32, 6511-6518 (2004).

107. Beck, H. C. et al. Quantitative Proteomic Analysis of Post-translational Modifications of Human Histones. Mol. Cell. Proteomics 5, 1314-1325 (2006).

108. Goudarzi, A. et al. Dynamic Competing Histone H4 K5K8 Acetylation and Butyrylation Are Hallmarks of Highly Active Gene Promoters. Mol. Cell 62, 169-180 (2016).

109. Hake, S. B. et al. Expression patterns and post-translational modifications associated with mammalian 
histone H3 variants. J. Biol. Chem. 281, 559-568 (2006).

110. Tan, M. et al. Identification of 67 histone marks and histone lysine crotonylation as a new type of histone modification. Cell 146, 1016-1028 (2011).

111. Moniruzzaman, M., Martinez-Gutierrez, C. A., Weinheimer, A. R. \& Aylward, F. O. Dynamic genome evolution and complex virocell metabolism of globally-distributed giant viruses. Nat. Commun. 11, 1$11(2020)$.

112. Punta, M. et al. The Pfam protein families database. Nucleic Acids Res. 40, D290-301 (2012).

113. Eddy, S. R. Accelerated profile HMM searches. PLoS Comput. Biol. 7, e1002195 (2011).

114. Buchfink, B., Xie, C. \& Huson, D. H. Fast and sensitive protein alignment using DIAMOND. Nat. Methods 12, 59-60 (2015).

115. Enright, A. J., Van Dongen, S. \& Ouzounis, C. A. An efficient algorithm for large-scale detection of protein families. Nucleic Acids Res. 30, 1575-1584 (2002).

116. Steenwyk, J. L., Buida, T. J., Li, Y., Shen, X.-X. \& Rokas, A. ClipKIT: A multiple sequence alignment trimming software for accurate phylogenomic inference. PLOS Biol. 18, e3001007 (2020).

117. Minh, B. Q., Nguyen, M. A. T. \& von Haeseler, A. Ultrafast approximation for phylogenetic bootstrap. Mol. Biol. Evol. 30, 1188-95 (2013).

118. Grau-Bové, X. \& Sebé-Pedrós, A. Orthology Clusters from Gene Trees with Possvm. Mol. Biol. Evol. 1-5 (2021) doi:10.1093/molbev/msab234.

119. Huerta-Cepas, J., Dopazo, H., Dopazo, J. \& Gabaldón, T. The human phylome. Genome Biol. 8, R109 (2007).

120. Huerta-Cepas, J., Serra, F. \& Bork, P. ETE 3: Reconstruction, Analysis, and Visualization of Phylogenomic Data. Mol. Biol. Evol. 33, 1635-1638 (2016).

121. Csürös, M. \& Miklós, I. A Probabilistic Model for Gene Content Evolution with Duplication, Loss, and Horizontal Transfer BT - Research in Computational Molecular Biology. in (eds. Apostolico, A., Guerra, C., Istrail, S., Pevzner, P. A. \& Waterman, M.) 206-220 (Springer Berlin Heidelberg, 2006).

122. Csurös, M. Count: evolutionary analysis of phylogenetic profiles with parsimony and likelihood. Bioinformatics 26, 1910-2 (2010).

123. Gansner, E. R. \& North, S. C. An open graph visualization system and its applications to software engineering. Softw. Pract. Exp. 30, 1203-1233 (2000).

124. Fu, L., Niu, B., Zhu, Z., Wu, S. \& Li, W. CD-HIT: accelerated for clustering the next-generation sequencing data. Bioinformatics 28, 3150-3152 (2012).

125. Jombart, T., Balloux, F. \& Dray, S. adephylo: new tools for investigating the phylogenetic signal in biological traits. Bioinformatics 26, 1907-1909 (2010).

126. Wells, J. N. \& Feschotte, C. A Field Guide to Eukaryotic Transposable Elements. Annu. Rev. Genet. 54, annurev-genet-040620-022145 (2020).

127. Storer, J., Hubley, R., Rosen, J., Wheeler, T. J. \& Smit, A. F. The Dfam community resource of transposable element families, sequence models, and genome annotations. Mob. DNA 12, 2 (2021).

128. Camacho, C. et al. BLAST+: architecture and applications. BMC Bioinformatics 10, 421 (2009).

129. Patro, R., Duggal, G., Love, M. I., Irizarry, R. A. \& Kingsford, C. Salmon provides fast and bias-aware quantification of transcript expression. Nat. Methods 14, 417-419 (2017).

130. Quinlan, A. R. \& Hall, I. M. BEDTools: a flexible suite of utilities for comparing genomic features. Bioinforma. 26, 841-842 (2010).

131. Anisimova, M., Gil, M., Dufayard, J.-F., Dessimoz, C. \& Gascuel, O. Survey of Branch Support 
bioRxiv preprint doi: https://doi.org/10.1101/2021.11.30.470311; this version posted December $1,2021$. The copyright holder for this

preprint (which was not certified by peer review) is the author/funder, who has granted bioRxiv a license to display the preprint in perpetuity. It is made available under aCC-BY-NC 4.0 International license.

Methods Demonstrates Accuracy, Power, and Robustness of Fast Likelihood-based Approximation Schemes. Syst. Biol. 60, 685-699 (2011).

132. Bodenhofer, U., Bonatesta, E., Horejš-Kainrath, C. \& Hochreiter, S. msa: an R package for multiple sequence alignment. Bioinformatics 31, 3997-3999 (2015). 